\title{
One-dimensional Numerical Model of Transient Discharges in Air of a Spatial Plasma Ignition Device
}

by

Florin N. Saceleanu

A thesis submitted to the Faculty of Graduate and Postdoctoral Affairs in partial fulfillment of the requirements for the degree of

Master of Applied Science

in

Mechanical and Aerospace Engineering

Carleton University

Ottawa, Ontario

(C) 2013, Florin N. Saceleanu 
Library and Archives

Canada

Published Heritage

Branch

395 Wellington Street

Ottawa ON K1A ON4

Canada
Bibliothèque et

Archives Canada

Direction du

Patrimoine de l'édition

395 , rue Wellington

Ottawa ON K1A ON4

Canada
Your file Votre référence

ISBN: 978-0-494-94651-0

Our file Notre référence

ISBN: 978-0-494-94651-0
NOTICE:

The author has granted a nonexclusive license allowing Library and Archives Canada to reproduce, publish, archive, preserve, conserve, communicate to the public by telecommunication or on the Internet, loan, distrbute and sell theses worldwide, for commercial or noncommercial purposes, in microform, paper, electronic and/or any other formats.

The author retains copyright ownership and moral rights in this thesis. Neither the thesis nor substantial extracts from it may be printed or otherwise reproduced without the author's permission.
AVIS:

L'auteur a accordé une licence non exclusive permettant à la Bibliothèque et Archives Canada de reproduire, publier, archiver, sauvegarder, conserver, transmettre au public par télécommunication ou par l'Internet, prêter, distribuer et vendre des thèses partout dans le monde, à des fins commerciales ou autres, sur support microforme, papier, électronique et/ou autres formats.

L'auteur conserve la propriété du droit d'auteur et des droits moraux qui protege cette thèse. $\mathrm{Ni}$ la thèse ni des extraits substantiels de celle-ci ne doivent être imprimés ou autrement reproduits sans son autorisation.
In compliance with the Canadian Privacy Act some supporting forms may have been removed from this thesis.

While these forms may be included in the document page count, their removal does not represent any loss of content from the thesis.
Conformément à la loi canadienne sur la protection de la vie privée, quelques formulaires secondaires ont été enlevés de cette thèse.

Bien que ces formulaires aient inclus dans la pagination, il n'y aura aucun contenu manquant. 


\section{Abstract}

This thesis examines the modes of discharge of a plasma ignition device. Oscilloscope data of the discharge voltage and current are analyzed for various pressures in air at ambient temperature. It is determined that the discharge operates in 2 modes: a glow discharge and a postulated streamer discharge. Subsequently, a 1-dimensional fluid simulation of plasma using the finite volume method (FVM) is developed to gain insight into the particle kinetics. Transient results of the simulation agree with theories of electric discharges; however, quasi-steady state results were not reached due to high diffusion time of ions in air.

Next, an ordinary differential equation (ODE) is derived to understand the discharge transition. Simulated results were used to estimate the voltage waveform, which describes the ODE's forcing function; additional simulated results were used to estimate the discharge current and the ODE's non-linearity. It is found that the ODE's non-linearity increases exponentially for capacitive discharges. It is postulated that the non-linearity defines the mode transition observed experimentally.

The research is motivated by Spatial Plasma Discharge Ignition (SPDI), an innovative ignition system postulated to increase combustion efficiency in automobile engines for up to $9 \%$. The research thus far can only hypothesize SPDI's benefits on combustion, based on the literature review and the modes of discharge. 


\section{Acknowledgements}

I would like to thank my supervisor, Dr. Ronald Miller, for giving me the opportunity, guidance and financial support to complete this thesis. I would also like to thank Mr.

Glen Clarke and Dr. Alex Plotnikov, from Sphenic Technologies Inc., for providing a SPDI device and financial support for this research.

In particular, I am grateful to Dr. Tanvir Farouk, from University of South Carolina, for the discussions and guidelines regarding plasma modeling.

I would also like to acknowledge my family for their encouragement and additional financial support whenever necessary. 


\section{Table of Contents}

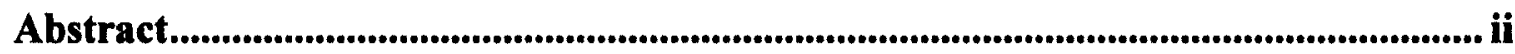

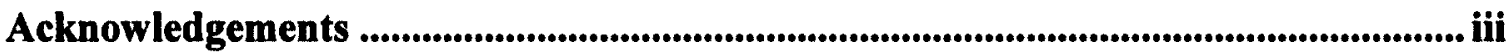

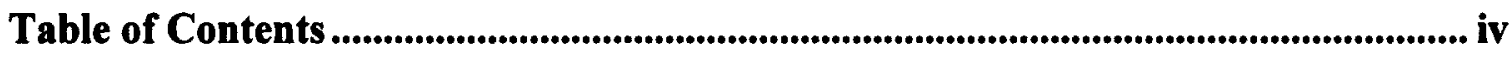

List of Tables ................................................................................................................................ $x$

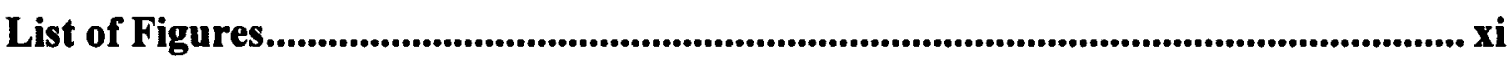

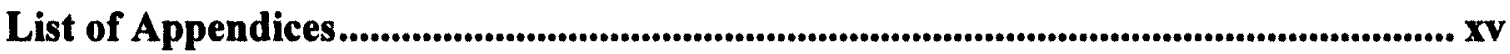

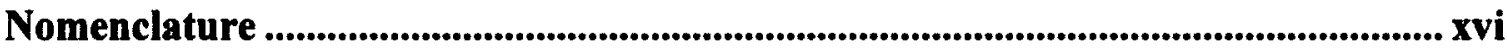

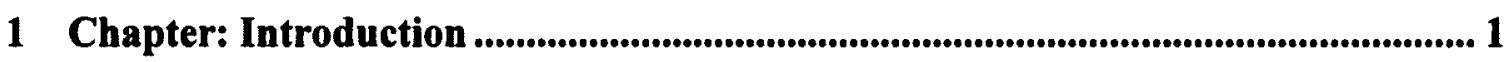

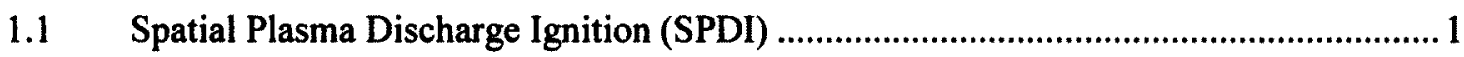

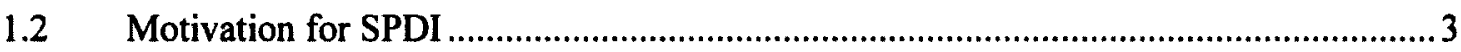

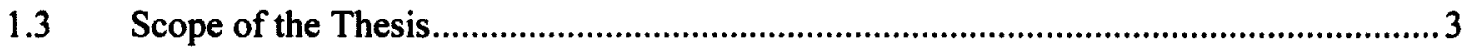

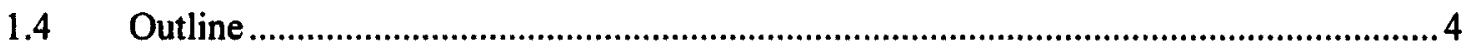

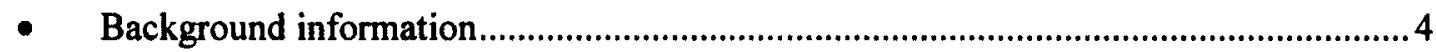

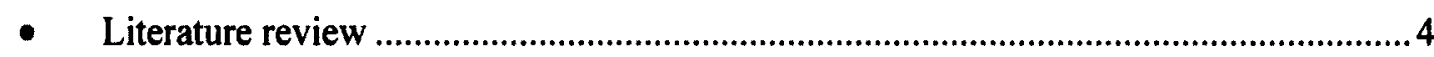

- Analysis of SPDI experiments and relevant assumptions ......................................... 4

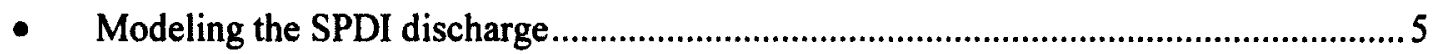

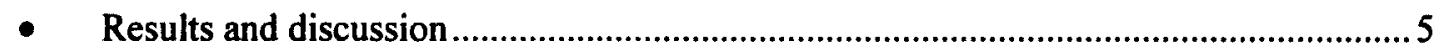

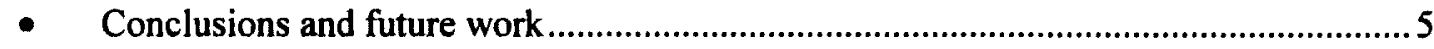

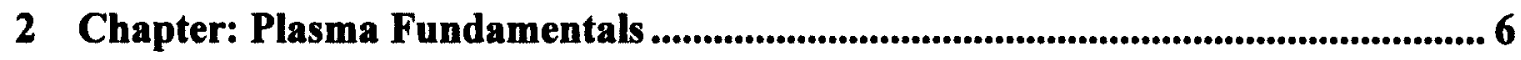

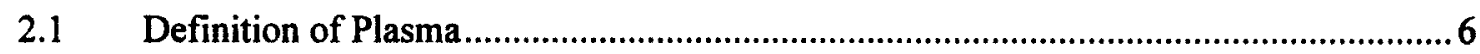

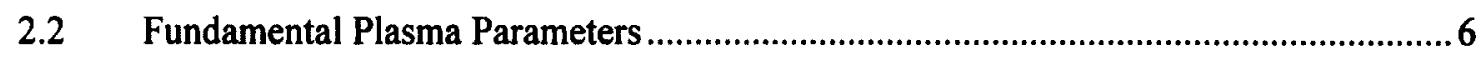

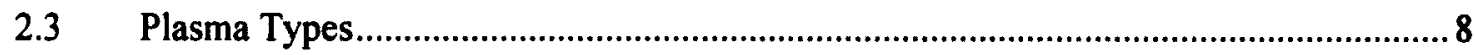




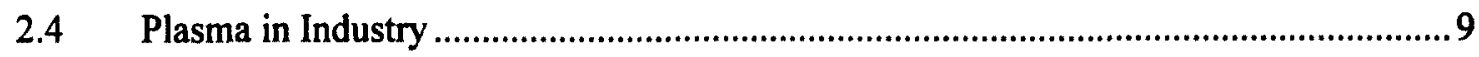

2.5 Parameters Describing Plasma Behavior..............................................................10

2.5.1 Particle collisions and the effect of electric field ................................................... 10

2.5.2 Free and ambipolar diffusion ......................................................................... 12

2.5.3 Degree of quasi-neutrality and boundary surface interaction .................................. 13

3 Chapter: Thermodynamics and Kinetics in Plasma ......................................................... 15

3.1 Overview of Statistical Thermodynamics ………….................................................. 15

3.2 The Internal Energies of Diatomic Molecules.............................................................. 16

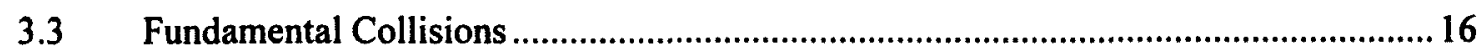

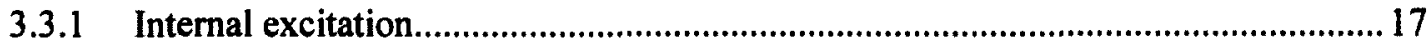

3.3.2 Direct impact ionization .................................................................................

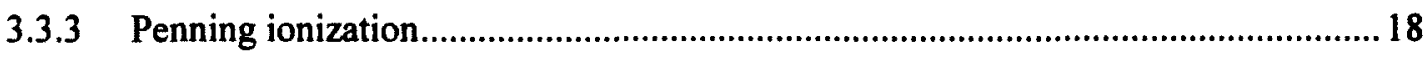

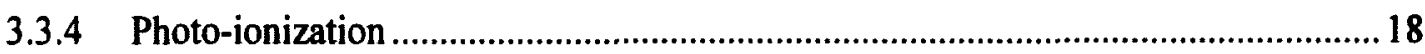

3.3.5 Electron attachment....................................................................................... 18

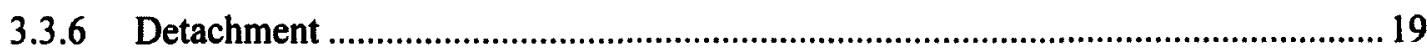

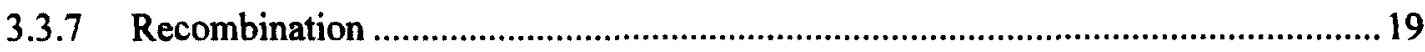

3.4 Kinetics Applicable to Plasma ................................................................................. 19

3.4.1 Boltzmann kinetic equation............................................................................... 19

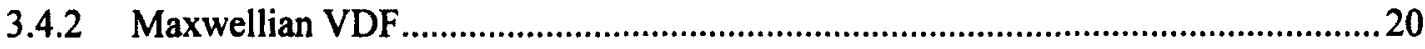

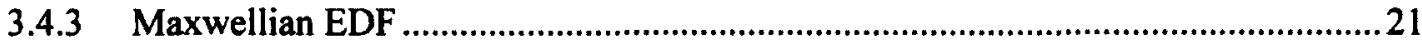

3.4.4 Transport and rate coefficients from the EDF .....................................................22

3.5 Importance of Statistics in Particle Reactions ……………….....................................2 23

3.6 Electromagnetics in Plasma Media..............................................................................23

3.6.1 Electromagnetic wave theory ................................................................................24

3.6.2 Electromagnetic propagation in plasma medium ……............................................24

3.6.3 Oscillating electromagnetic fields .......................................................................25 


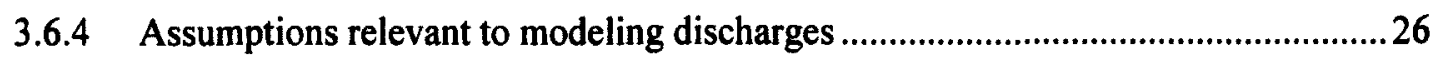

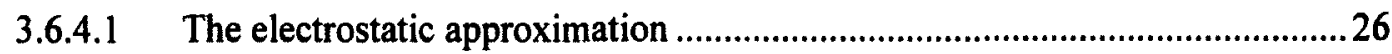

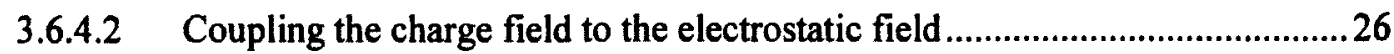

4 Chapter: Overview of the Electric Discharge and its Modeling............................. 28

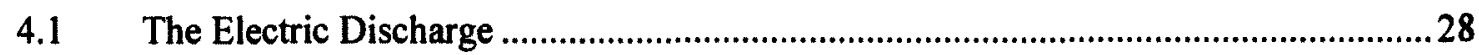

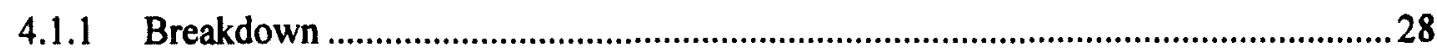

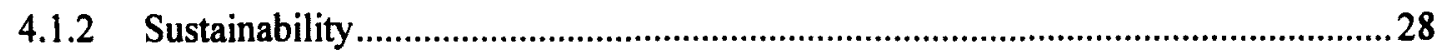

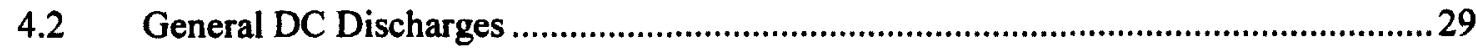

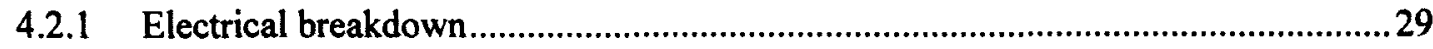

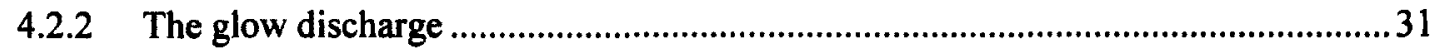

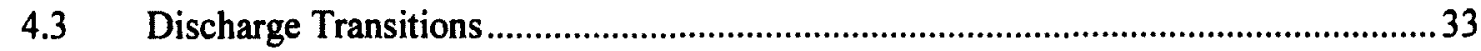

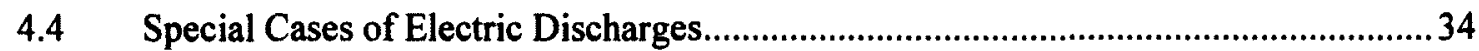

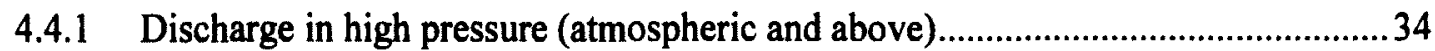

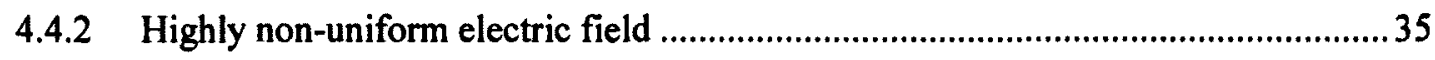

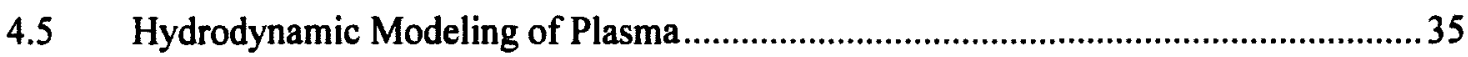

4.5.1 Local field approximation and its limitations ..................................................... 37

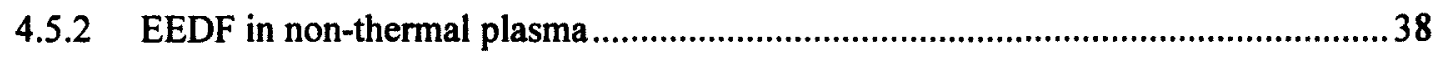

5 Chapter: Literature Review ................................................................................ 39

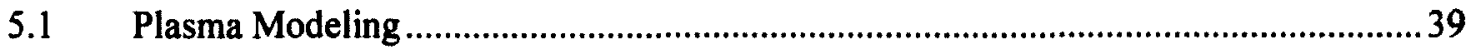

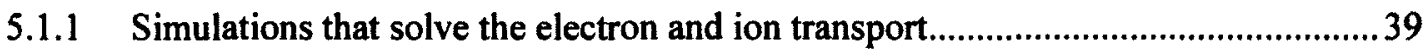

5.1.2 Simulations that solve the electron, ion and electron energy transport....................40

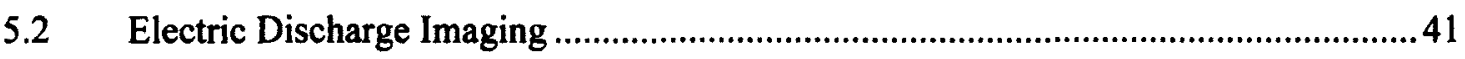

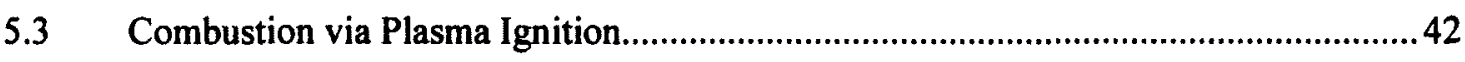

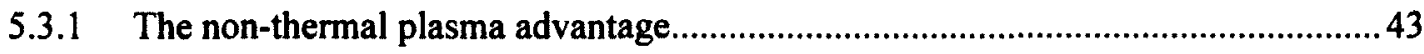

5.3.2 Relevant combustion experiments involving non-thermal plasma .......................44 
6 Chapter: Experimental Observations of SPDI............................................................. 49

6.1 The Experimental Setup ......................................................................................

6.2 The Plasma Support Phase …………………….................................................. 49

$6.3 \quad$ SPDI Supported Combustion .................................................................................5

6.3.1 Plasma support phase under low/high load and duration ........................................50

6.4 SPDI in Pressurized Air....................................................................................52

6.4.1 The 2 discharge modes ........................................................................................5

6.4.2 Discharge imaging …………………............................................................... 54

6.5 Discharge Power Analysis................................................................................56

6.5.1 Power factor of the plasma support phase .............................................................59

6.6 SPDI Comparison to Experiments in the Literature ...................................................60

6.6.1 The atmospheric pressure plasma jet (APPJ) ……………………….......................61

$6.7 \quad$ Remarks on experimental testing ……………...................................................62

6.8 Fundamental Assumptions relevant to Modeling SPDI ................................................63

6.8.1 High air pressure .................................................................................................6

6.8.2 The electrostatic approximation in SPDI plasma ......................................................63

6.8.3 Polarization field in SPDI plasma ........................................................................6

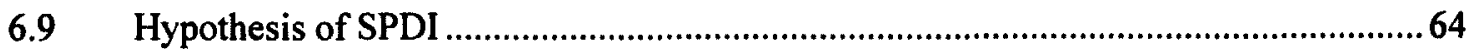

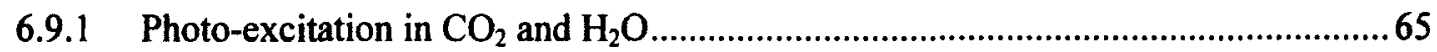

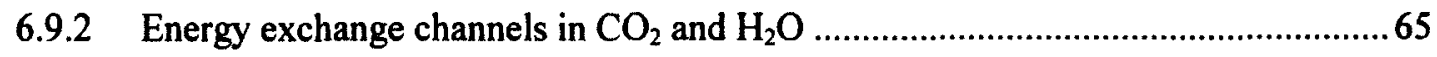

7 Chapter: Plasma Model Formulation .................................................................................6 67

7.1 Purpose of the Model and the Modeling Approach......................................................67

7.2 The 1D Approximation and Importance of 3D effects ................................................67

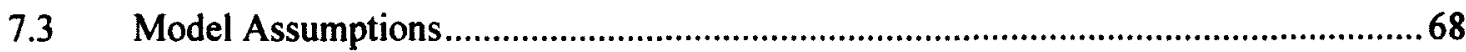

$7.4 \quad$ Governing Equations ...........................................................................................69

$7.5 \quad$ Transport and Rate Coefficients ...........................................................................72 


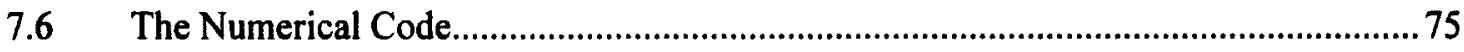

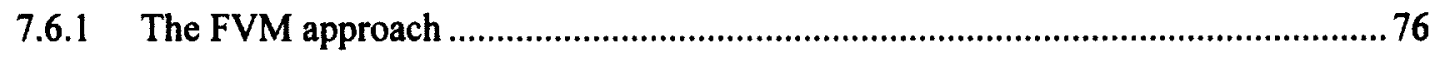

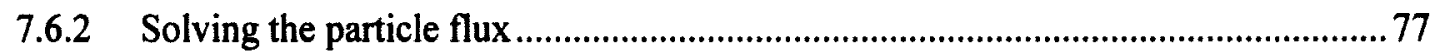

7.6.2.1 Exponential scheme for drift-diffusion................................................... 77

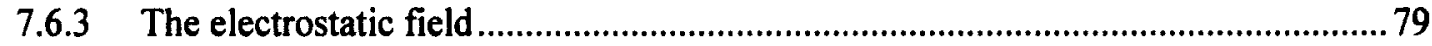

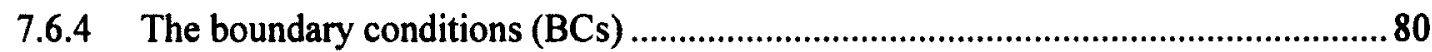

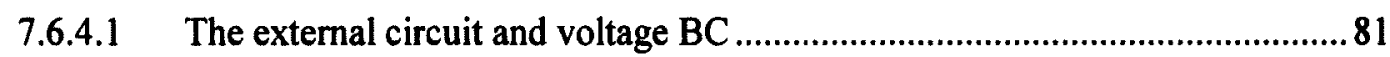

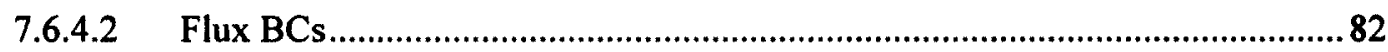

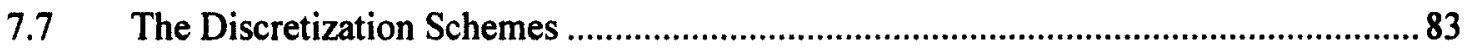

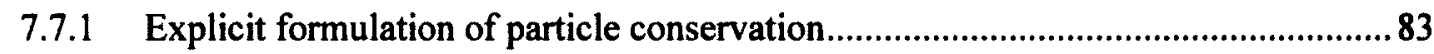

7.7.2 Semi and fully-implicit formulations of particle conservation ............................... 84

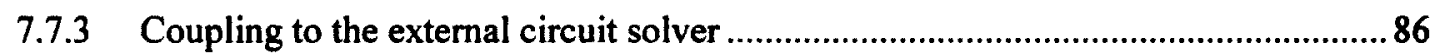

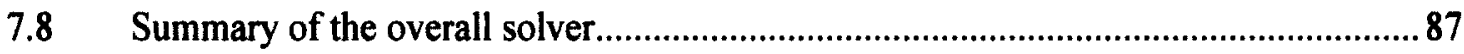

7.8.1 The link between the model and experimental SPDI discharges in air ...................89

8 Chapter: ODE Interpretation of SPDI Discharge ........................................... 90

8.1 Simple Interpretation of a plasma bounded by the electrodes ...................................91

8.2 Assumptions and Derivations for deriving the ODE .........................................91

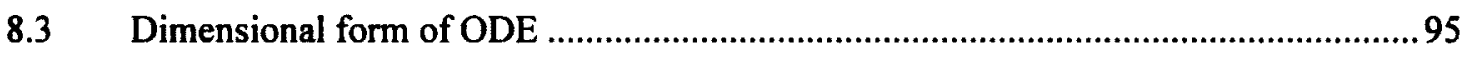

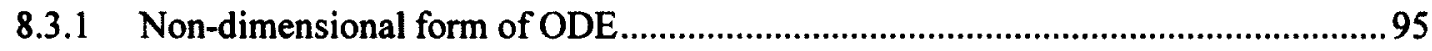

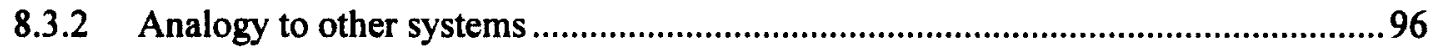

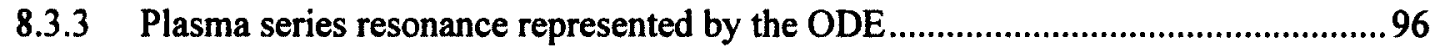

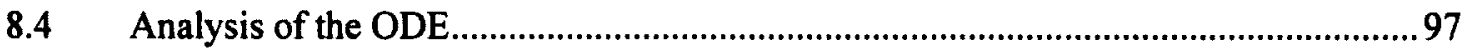

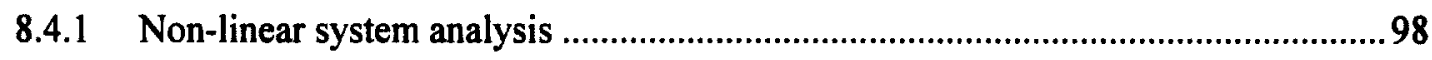

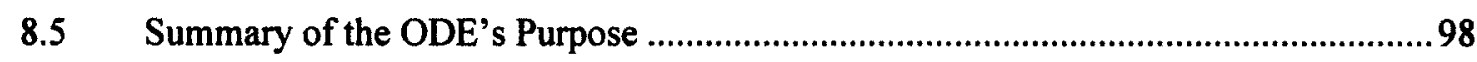

9 Chapter: Results and Discussion of the Plasma Model and the ODE................. 99

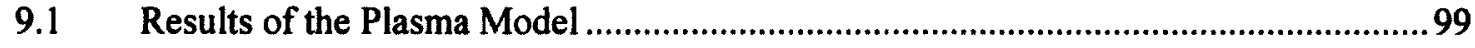


9.1.1 Transient plasma during breakdown

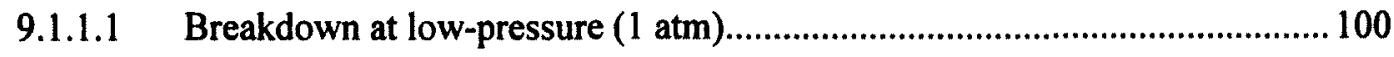

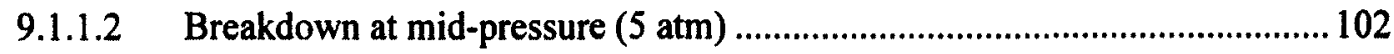

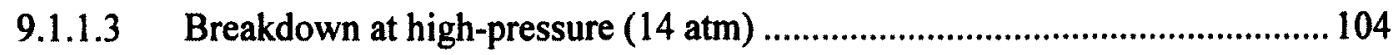

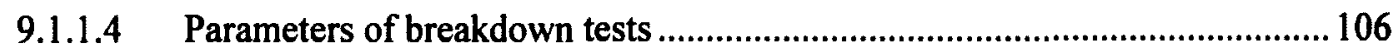

9.1.1.5 Relation between the ionization wave velocity and pressure ........................ 106

9.1.2 Plasma distribution of RF and DC discharges ............................................. 107

9.1.2.1 Simulated RF discharges at atmospheric pressure ................................... 107

9.1.2.2 Plasma distributions in transient glow DC discharges ...............................113

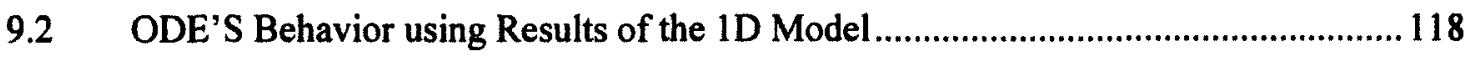

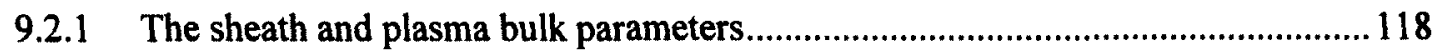

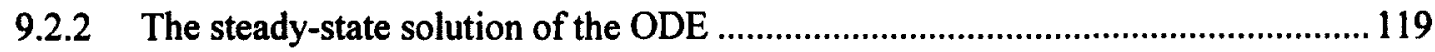

9.2.3 Describing function of the non-linear sheath voltage .................................... 120

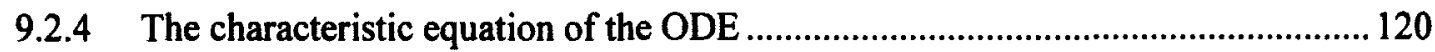

9.2.5 Results of the sheath's voltage dependence on the phase-shift ............................ 121

10 Chapter: Concluding Remarks.......................................................................... 123

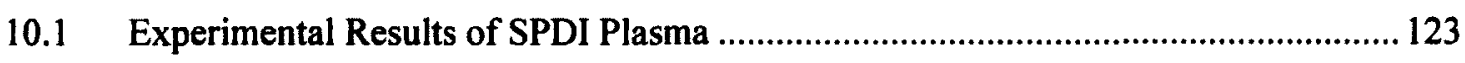

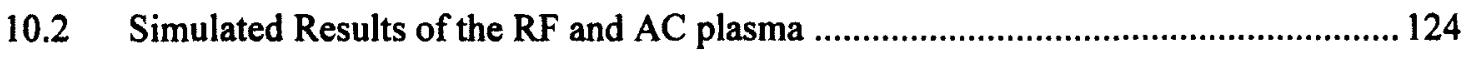

10.3 Limitations and Improvements of the Plasma Model.......................................... 125

10.4 Improvements in the Computational Time of the Plasma Model ..............................126

$10.5 \quad$ Future Work regarding SPDI Assisted Combustion............................................ 127

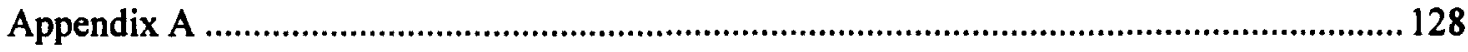

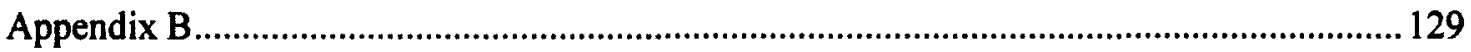

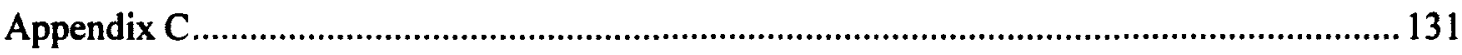

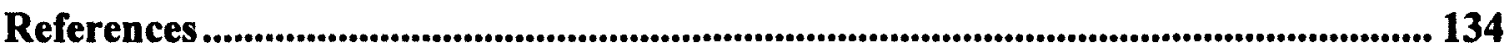




\section{List of Tables}

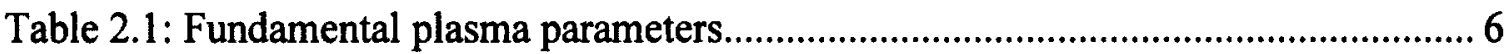

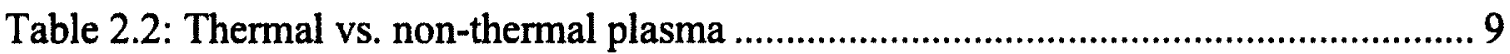

Table 9.1: Summary of results for the breakdown simulations ...................................... 106

Table 9.2: Summary of the discharge results for the transient DC simulations ............. 118

Table 9.3: Summary of parameters used in the ODE .................................................. 119 


\section{List of Figures}

Figure 1.1: Voltage-current phases of SPDI ............................................................. 2

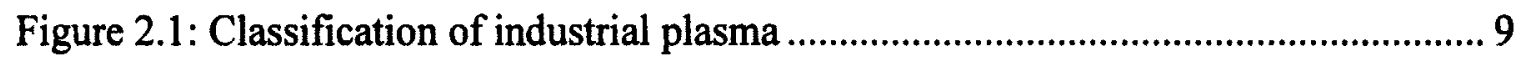

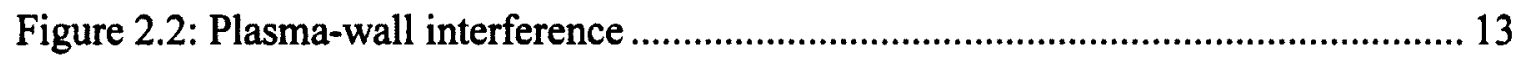

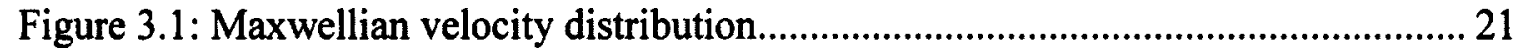

Figure 3.2: Maxwellian and Druyvesteyn energy distributions......................................... 22

Figure 4.1: Characteristics of general DC discharges...................................................... 29

Figure 4.2: Photograph of a spark discharge ……………............................................. 30

Figure 4.3: Physics of the DC glow discharge ................................................................. 32

Figure 4.4: Photograph of a glow discharge ……......................................................... 32

Figure 5.1: The first electron avalanche observed ..................................................... 41

Figure 5.2: Schlieren photograph of streamer discharges............................................. 42

Figure 5.3: $\mathrm{OH}$ radical production under non-thermal plasma ........................................... 44

Figure 5.4: Turbulent flame with non-thermal plasma ignition......................................... 45

Figure 5.5: Combustion pressure history for spark and corona ignition............................ 46

Figure 5.6: Flame propagation under DBD discharge ……............................................ 46

Figure 5.7: a) Schlieren protographs of the flame propagation, and b) Pressure

combustion history of spark and corona ignition............................................................ 48

Figure 6.1: Overview of the experimental setup for testing SPDI (NOTE the highly nonuniform electrode geometry).

Figure 6.2: Oscilloscope data of SPDI ignition at low engine load

NOTE: the last pulse on the TOP figure is the coil discharge (around $2.645 \cdot 10^{4} \mu \mathrm{s}$ ) $\ldots . . .51$ 
Figure 6.3: Oscilloscope data of SPDI ignition at high engine load

NOTE: the last pulse on the TOP figure is the coil discharge (around $6.12 \cdot 10^{4} \mu \mathrm{s}$ ) $\ldots \ldots . .51$

Figure 6.4: Oscilloscope data of the first mode of discharge in air at atmospheric pressure

Figure 6.5: Oscilloscope data of the second mode of discharge in air at 120 psi gauge

pressure 54

Figure 6.6: Sample image of the breakdown produced by SPDI................................... 54

Figure 6.7: Sample image of the glow discharge produced by SPDI ............................... 55

Figure 6.8: Average discharge power in the first discharge mode for the pressures tested

(Error bars show 1 standard deviation for the 8 ignition events)..................................... 56

Figure 6.9: Sample voltage-current hysteresis of the first mode, at atmospheric pressure

Figure 6.10: Average discharge power in the second discharge mode for the pressures tested (Error bars shows 1 standard deviation for the 8 ignition events). 57 Figure 6.11: Sample voltage-current hysteresis of the second mode, at 120 psi gauge pressure 58

Figure 6.12: Average discharge power at $110 \mathrm{psi}$ gauge pressure, illustrating both modes of discharge (Error bars show 1 standard deviation for the 8 ignition events) 58 Figure 6.13: Average real power of the plasma support phase of SPDI for the pressures tested 59

Figure 6.14: Average power factors of SPDI for the pressures tested...............................6 60

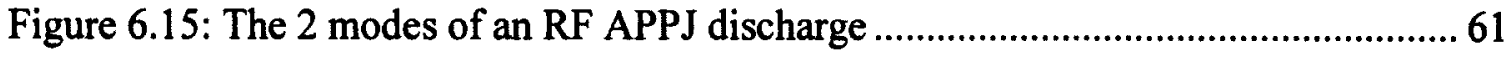

Figure 6.16: Hypothesis for excitation of the flame front using SPDI ............................. 64 


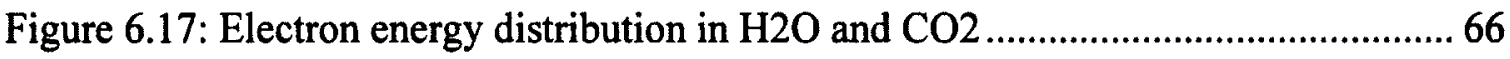

Figure 7.1: Sample plot of the reduced electron mobility ................................................ 73

Figure 7.2: Sample plot of the total rate of electron energy loss due to inelastic collisions

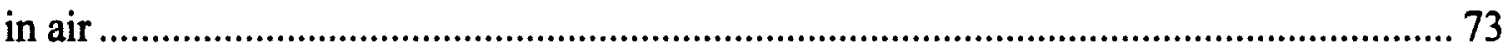

Figure 7.3: Spatial discretization via the staggered grid .................................................... 76

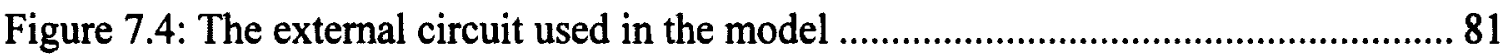

Figure 7.5: Flowchart of the overall plasma solver, implemented in Matlab ....................88

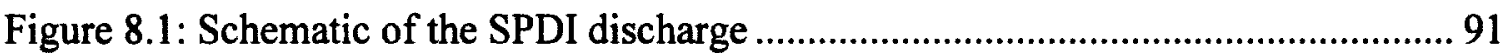

Figure 9.1a: Space charge and electric field distributions for breakdown at $1 \mathrm{~atm} . \ldots \ldots . .100$ Figure 9.1b: Space average electron density and discharge voltage-current for breakdown at $1 \mathrm{~atm}$ Figure 9.2: Breakdown current, and time and space averaged electron density for increasing number of nodes ...................................................................................... 102 Figure 9.3a: Space charge and electric field distributions for breakdown at $5 \mathrm{~atm} . \ldots . . . .103$ Figure 9.3b: Space average electron density and discharge voltage-current for breakdown at $5 \mathrm{~atm}$ 103

Figure 9.4a: Space charge and electric field distributions for breakdown at 14 atm...... 104 Figure 9.4b: Space average electron density and discharge voltage-current for breakdown at 14 atm 105

Figure 9.5a: Number density of species of the RF discharge at $1 \mathrm{~atm}$, averaged over the 10th cycle 108

Figure 9.5b: Spatial space charge, electric field, and electron temperature of the RF discharge at $1 \mathrm{~atm}$, averaged over the 10th cycle 109 
Figure 9.5c: Discharge voltage and current of the RF discharge at $1 \mathrm{~atm}$, approaching the 10th cycle 109

Figure 9.6a: Number density of species of the RF discharge at $1 \mathrm{~atm}$, averaged over the 100th cycle 110

Figure 9.6b: Spatial space charge, electric field, and electron temperature of the RF discharge at $1 \mathrm{~atm}$, averaged over the 100th cycle

Figure 9.6c: Discharge voltage and current of the RF discharge at $1 \mathrm{~atm}$, approaching the 100 th cycle

Figure 9.7a: Number density of plasma species for the transient DC glow at $1 \mathrm{~atm} \ldots . . .113$ Figure 9.7b: Spatial space charge, electric field, and electron temperature for the transient

$\mathrm{DC}$ glow at $1 \mathrm{~atm}$ 114

Figure 9.7c: Discharge voltage and current for the transient DC glow at $1 \mathrm{~atm}$ 115

Figure 9.8a: Number density of plasma species for the transient DC glow at $5 \mathrm{~atm} . \ldots . .115$ Figure 9.8b: Spatial space charge, electric field, and electron temperature for the transient $\mathrm{DC}$ glow at $5 \mathrm{~atm}$ 116

Figure 9.8c: Discharge voltage and current for the transient $\mathrm{DC}$ glow at $5 \mathrm{~atm}$ 117 Figure 9.9: Describing function of the sheath voltage for hypothetical discharges at SPDI's frequency 


\section{List of Appendices}

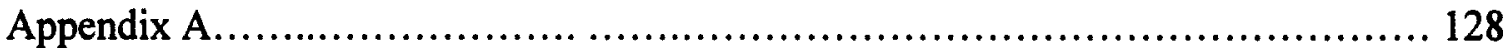

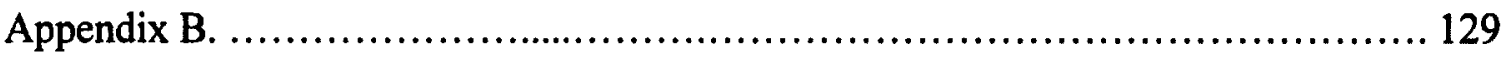

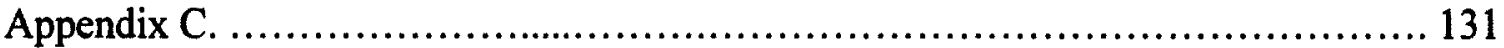




\section{Nomenclature}

\section{Acronyms}

APPJ

Atmospheric Pressure Plasma Jet

$\mathrm{BC}$

Boundary Condition

(E)EDF

(Electron) Energy Distribution Function

FVM

Finite Volume Method

ODE

Ordinary Differential Equation

SPDI Spatial Plasma Discharge Ignition

VDF

Velocity Distribution Function

\section{Symbols}

d

inter-electrode gap distance

n

number density of a charged particle

D

diffusion coefficient

$N$

number density of neutral molecules

$\mathbf{P}$

pressure

$\mathrm{T}$

temperature

V

Voltage

E Electric field vector

F Transport flux vector

$\omega_{p} \quad$ plasma frequency

$\omega_{\text {SPDI }}$

SPDI frequency 


\section{Greek symbols}

$\alpha$

$\beta$

$\varepsilon$

$\lambda$

$\gamma$

$\eta$

$\mu$

$\sigma$

$\vartheta$

$\Phi$

\section{Subscripts}

e

i

n

$\mathrm{p}$

$\varepsilon$

en

\section{Superscripts}

k

time step ionization coefficient

attachment coefficient

permittivity

mean free path

secondary electron emission coefficient

recombination coefficient

mobility

plasma conductivity

collision frequency

current-voltage phase-shift electron

spatial step

negative ion

positive ion

mean electron energy

electron-neutral 


\section{Chapter: Introduction}

Hydrocarbon combustion is an essential element of modern industry. In particular, nearly all transportation depends on the combustion process within the internal combustion (IC) engine. Most automobile engines have a spark ignition system to initiate the burning process. Intensive studies have been done on the fuel mixtures, airflow turbulence, and engine geometry, for improving the fuel consumption and reduce the pollutant emissions. However, there is significantly less research on the spark ignition system.

The conventional spark ignition of the IC engine produces a short and high temperature arc discharge to initiate the flame kernel. Yet over the past decade, ignition via nonthermal plasma sources has shown higher fuel efficiency and enhanced flame propagation over the arc discharge. The term non-thermal ignition refers to the non-equilibrium between the gas and electron temperatures, or specifically, weak exchange of kinetic energy between the gas molecules and electrons.

\subsection{Spatial Plasma Discharge Ignition (SPDI)}

SPDI is an innovative ignition system developed by Sphenic Technologies for improving combustion efficiency of automobile engines. It is triggered by an audio-frequency voltage pulse generator and uses a commercial spark plug with a gap of approximately 1 $\mathrm{mm}$. The manufacturer claims up to $9 \%$ lower fuel consumption and reduced $\mathrm{NO}_{\mathrm{x}}$ over conventional spark ignition.

A typical voltage-current plot of SPDI in atmospheric air is shown in Figure 1.1. SPDI discharge consists of 3 consecutive phases, which are user programmable through the configuration software. These phases are labeled in Figure 1.1 and described briefly here. 


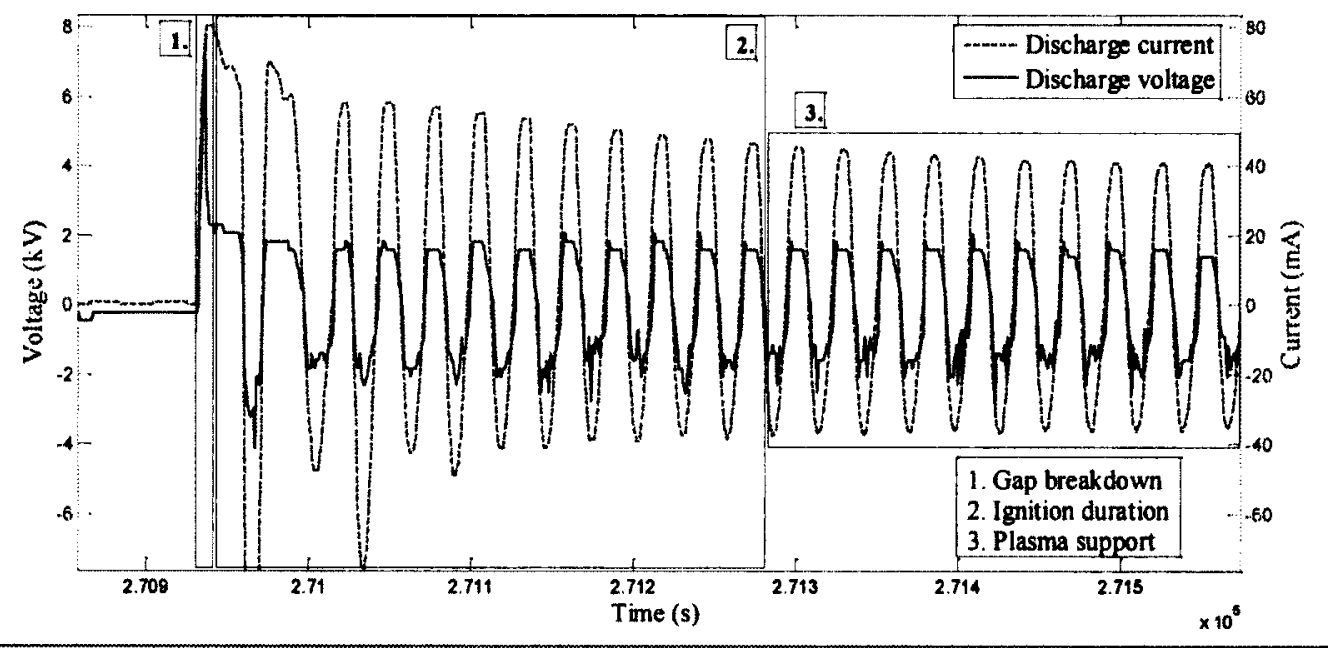

Figure 1.1: Voltage-current phases of SPDI

NOTE: duration of the $2^{\text {nd }}$ and $3^{\text {rd }}$ phase is not to scale

1. Gap breakdown

The gap breakdown phase is characterized by the breakdown current (or coil charge duration), which is set in the interface software. Breakdown ensures high electrical conductivity between the electrodes, which is necessary to sustain the subsequent non-thermal plasma.

2. Ignition duration

The ignition duration period provides energy to stabilize the flame kernel. This energy is set in the interface by providing the duration and power. The ignition power is controlled by the duty cycle of the circuit transistor.

3. Plasma support

The plasma support phase produces non-thermal plasma on a programmable time and power scale, to support the flame propagation. The plasma power is controlled by the duty cycle of the circuit transistor. The plasma support phase operates at approximately $35.7 \mathrm{kHz}$ frequency, and is the focus of this thesis. 


\subsection{Motivation for SPDI}

Spatial Plasma Discharge Ignition was developed to promote the combustion process in spark ignition engines. Unlike a conventional spark ignition, which produces a very hot thermal plasma arc of approximately $1 \mathrm{~ms}$ in duration, SPDI produces cold non-thermal plasma for a period of 2 to $4 \mathrm{~ms}$. SPDI is superior to a conventional spark ignition, according to the hypothesis that the discharge energy is better distributed to the combustible mixture. This hypothesis is discussed in Chapters 5 and 6.

\subsection{Scope of the Thesis}

The objective of this thesis is to investigate the discharge modes of SPDI's plasma support phase. Oscilloscope data of the discharge voltage and current are analyzed and operation regimes are defined. A theoretical model is then developed to link plasma kinetics of electric discharges to experimental observations in SPDI. The modes of discharge are verified with theories and comparison to recent publications.

It should be noted that simulations presented in this thesis are only relevant to parallel plate geometry since the 3D geometry of spark plugs is ignored. For modeling purposes, we assume that the discharge propagates only axially between the electrodes.

Furthermore, the results of this thesis will not connect the SPDI discharge to combustion processes directly. However, experimental observations and theoretical results approximating SPDI confirm its non-equilibrium nature, the benefits of which are presented in the literature review. The benefits of SPDI supported combustion are postulated in Chapter 6. 


\subsection{Outline}

This thesis starts with a review of the fundamentals of plasma kinetics and thermodynamics, and the coupling to electromagnetics. Afterwards, relevant literature on plasma modeling and combustion ignition via non-thermal plasma is discussed. Next, experimental observations of SPDI are presented, followed by 1D modeling of the discharge.

\section{- Background information}

Chapters 2 and 3 outline the plasma types, particle distributions, plasma response to electromagnetic fields, and particle collisions relevant at atmospheric or higher pressure. An overview of electric discharges and discharge instabilities are described in Chapter 4. The principles within these background chapters are essential to understanding plasma modeling.

\section{- Literature review}

The literature review presented in Chapter 5 consists of two topics. Firstly, fluid modeling of plasma is introduced, and results of simulations in literature are discussed. Secondly, experimental results of combustion via non-thermal plasma ignition are presented. These experimental data indicate more efficient combustion than conventional spark ignitions. A hypothesis of SPDI is made on a similar basis to these results.

\section{- Analysis of SPDI experiments and relevant assumptions}

Oscilloscope data of the discharge voltage and current in SPDI are analyzed and discussed in Chapter 6. The discharge characteristics are evaluated during combustion and ignition in pressurized air at ambient temperature. The main purpose of this chapter is to determine the discharge power of the plasma states. Optical imaging of the plasma 
states in air are also presented, and compared to literature. The end of the chapter outlines essential assumptions relevant to SPDI for modeling purposes.

\section{- Modeling the SPDI discharge}

The emphasis of this thesis is to develop a 1-dimensional fluid model to simulate the SPDI discharge in air. This is presented in Chapter 7. The main assumption of this model is the highly non-thermal nature of plasma, such that air molecules are considered an infinite source at ambient temperature. The model is written in MATLAB using the Finite Volume Method (FVM). The transport and rate coefficients are determined from published semi-empirical and calculated data. The purpose of the 1D model is to determine the plasma species and electric field distributions along the axis of discharge.

In Chapter 8, a non-dimensional non-linear Ordinary Differential Equation (ODE) is developed to interpret the voltage-current behavior of SPDI. The purpose of the ODE is to analyze its non-linearity, then classify the plasma states of SPDI.

\section{- Results and discussion}

The first part of Chapter 9 presents simulation results for direct current (DC) breakdown, and DC/radio-frequency (RF) glow plasma in air using the 1D model. These results are compared to theories of electric discharges. The second part of Chapter 9 illustrates ODE results of the non-linearity for various discharge conditions. The operation regimes of the plasma states in pressurized air observed in Chapter 6 are identified.

\section{- Conclusions and future work}

Chapter 10 concludes with an overview of the limitations of the plasma model developed. Recommendations for future work involving plasma modeling and experimental testing of the SPDI discharge are also mentioned. 


\section{Chapter: Plasma Fundamentals}

This chapter presents the fundamentals of plasma. Relevant parameters are defined. The reader must have knowledge of these parameters prior to further reading into the thesis.

\subsection{Definition of Plasma}

Plasma is considered a fourth state of matter. It is a collection of ionized, dissociated and excited gas particles that is electrically neutral overall. Although microscopic collisions among plasma species (i.e. neutral molecules, ions, electrons) control their temporal distributions, transport and rate coefficients in plasma are usually computed from quasiequilibrium distributions.

\subsection{Fundamental Plasma Parameters}

The most important parameters of plasma (Akishev, 2007) are given in Table 2.1 below.

Table 2.1: Fundamental plasma parameters

\begin{tabular}{|l|l|l|}
\hline Parameter & Meaning & Importance \\
\hline $\begin{array}{l}\text { Reduced electric } \\
\text { field }^{1},(\mathrm{E} / \mathrm{N})\end{array}$ & $\begin{array}{l}\text { Ratio of electric field to gas } \\
\text { density or pressure }\end{array}$ & $\begin{array}{l}\text { Channels of energy transfer and } \\
\text { intensity of collisional processes } \\
\text { depend on E/N }\end{array}$ \\
\hline $\begin{array}{l}\text { Temperature } \\
\text { (T) }\end{array}$ & $\begin{array}{l}\text { Measure of the energy state } \\
\text { of a particle or system }\end{array}$ & $\begin{array}{l}\text { Indicates how energy is distributed } \\
\text { (kinetic, or internal forms) }\end{array}$ \\
\hline $\begin{array}{l}\text { Number density, } \\
\text { (n) }\end{array}$ & $\begin{array}{l}\text { Particle density (electrons, } \\
\text { ions, etc.) }\end{array}$ & Amount of species reacting \\
\hline $\begin{array}{l}\text { Degree of } \\
\text { ionization }\end{array}$ & $\begin{array}{l}\text { Density ratio of electrons to } \\
\text { gas molecules }\end{array}$ & $\begin{array}{l}\text { Determines the type of plasma (see } \\
\text { following section) }\end{array}$ \\
\hline
\end{tabular}

\footnotetext{
${ }^{1}$ Note that the unit for the reduced electric field is usually the Townsend $\left(1 \mathrm{Td}=10^{-17} \mathrm{Vcm}^{2}\right)$

${ }^{2}$ Note that the unit for the electron temperature is usually the electron-volt $(1 \mathrm{eV} \approx 11605 \mathrm{~K})$
} 
In quantum mechanics, the energy of a molecule consists of 3 internal forms of energy (rotational, vibrational, and electronic transition) and 1 external (translational). The total energy is the summation of all 4 energy degrees of freedom

$$
E=\sum_{i} E_{i}+E_{\text {trans }}
$$

where the $i$ subscript indicates an internal form of energy. Energy, under any of the forms above, is related to its temperature via the Boltzmann constant $k$,

$$
E_{j}=k T_{j}
$$

where the $j$ subscript indicates any of the 4 forms of energy. A temperature probe can only be sensitive to changes in translational (or kinetic) energy of molecules due to particle collisions. Hence internally excited states can only be determined via the emission spectra. Assuming that collisions between plasma particles ensure a most probable state of energy distribution, then the temperature of a plasma particle is equivalent to its average kinetic energy,

$$
T_{p a r}=\frac{2 \overline{E_{p a r}}}{3 k}
$$

where subscript par indicates a plasma particle (in the following, $e$ for electrons, $p$ for positive ions, and $n$ for negative ions), $\overline{E_{p a r}}$ represents the time-average kinetic energy, and the factor $2 / 3$ arises from the quasi-equilibrium Maxwellian distribution (Fridman, 2008). This distribution is discussed in detail in Chapter 3.

If a single temperature describes the energy state of a system such as plasma, then that system must be in thermal equilibrium among the 4 energy degrees of freedom. This is the case for thermal plasmas. However, factors such as the reduced electric field and pressure can cause non-equilibrium energy distributions, where the electron temperature 
is much higher than the gas temperature (hence the non-thermal plasmas). Note that gas temperature is strictly the temperature of the neutrally-charged gas molecules/atoms.

\subsection{Plasma Types}

Plasma is classified as thermal or non-thermal. There are 2 main factors that separate these plasma types: the channels of energy distribution, and the collision rate dependence.

In thermal plasmas, such as the arc discharge, the electron temperature is the same order of magnitude as the gas and ion temperatures. This is a condition of local thermodynamic equilibrium (Fridman \& Kennedy, 2004). Plasma under local thermodynamic equilibrium has low values of the reduced electric field. In this case, collisional rates are highly dependent on the gas temperature. Note that an ion can receive additional energy from an electric field due to its charge; hence the ion temperature is different than the gas temperature.

In non-thermal plasmas, such as the glow discharge, the electron temperature is orders of magnitude higher than the gas temperature, although the ion temperature is the same order as the gas temperature (Fridman \& Kennedy, 2004). In this case, there is no thermodynamic equilibrium and knowledge of the electron energy is very important. Formulation of the electron energy distribution function (EEDF) dictates the rates of collisional processes and thus the evolution of the plasma species. Table 2.2 summarizes the key features of thermal and non-thermal plasma. 
Table 2.2: Thermal vs. non-thermal plasma

\begin{tabular}{|l|l|l|}
\hline Plasma type & Temperature relation & Examples \\
\hline Thermal & $\mathrm{T}_{\mathrm{e}} \approx \mathrm{T}_{\text {internal }} \approx \mathrm{T}_{\text {gas }}$ & Arc discharge, thermonuclear, fusion \\
\hline Non-thermal & $\mathrm{T}_{\mathrm{e}} \gg \mathrm{T}_{\text {internal }} \geq \mathrm{T}_{\text {gas }}$ & Glow and corona discharges, microwave \\
\hline
\end{tabular}

The research of this thesis is on non-thermal plasma (specifically, the glow discharge). It should be noted that excited species play a key role in non-thermal plasmas. An excited species refers to a molecule or atom for which one of its internal energy states is higher than its ground state.

\subsection{Plasma in Industry}

Industrial plasma can be categorized by the electron density and temperature (its average

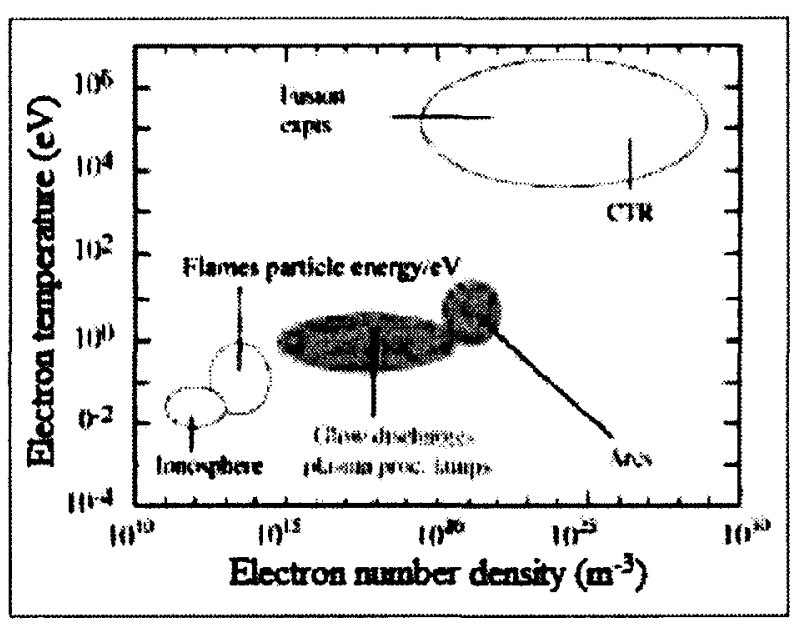

Figure 2.1: Classification of industrial plasma kinetic energy). Figure 2.1 (Braithwaite, 2000) shows the classification of common industrial plasma. The glow discharge is characterized by an electron density of $10^{15}$ to $10^{21} \mathrm{~m}^{-3}$ and electron temperature of $10^{-1}$ to $10^{2} \mathrm{eV}$. Considering that at STP, there are $2.5 \cdot 10^{25} \mathrm{~m}^{-3}$ molecules of air, the degree

of ionization of in glow discharges is on the order of $10^{-10}$ to $10^{-4}$. This separates nonthermal plasma from thermal plasma where full ionization is possible and electron temperatures reach $10^{3} \mathrm{eV}$ and higher. 


\subsection{Parameters Describing Plasma Behavior}

The most important aspects of plasma discharges are particle collisions, effects of the electric field, free and ambipolar diffusion, degree of quasi-neutrality, and plasma boundary surface interaction. Each of these is discussed in the following subsections.

\subsubsection{Particle collisions and the effect of electric field}

The collision cross section $\sigma(\varepsilon)$ is the interaction area of 2 colliding particles that results in a chemical reaction. Electron collisions with gas molecules are elementary in nonthermal plasmas, and their cross sections depend on the electron energy $\varepsilon$. For example, if the cross section for vibrational excitation of $\mathrm{N}_{2}$ via electron impact is maximized, one obtains the peak collision probability of an electron with $\mathrm{N}_{2}$ molecules, such that the density of vibrationally excited $\mathrm{N}_{2}$ molecules is maximized. However, collision cross sections can be smaller than the geometrical cross section if no chemical reaction occurs (Fridman, 2008). If the collision cross section is known, the mean free path can be determined from

$$
\lambda=\frac{1}{N 6(\varepsilon)} .
$$

The mean free path is equivalent to the average collision-free distance travelled by a particle, where the gas density is $\mathrm{N}$.

Motion of neutral and charged particles within plasma is described by their velocity fields. If pressure and temperature are known, thermal velocity can be determined. The average thermal velocity of gas molecules can be found by assuming the ideal gas law (Braithwaite, 2000) 


$$
P=N k T_{\text {gas }}
$$

Then, assuming a quasi-equilibrium distribution, the average thermal velocity of a gas molecule of mass $m$ is

$$
\bar{v}=\sqrt{\frac{3 k T_{g a s}}{m}} .
$$

For charged particles, the relevant velocities are thermal and drift within the plasma's electric field $\boldsymbol{E}$. The drift velocity is proportional to the particle's mobility $\mu$,

$$
v_{d}= \pm \mu E
$$

The \pm sign indicates the particle's charge (conventionally, a positive charge follows the direction of the electric field).

The ratio of a particle's velocity to its mean free path determines the collision frequency, 9, which is the number of collisions per unit time (Fridman, 2008). For neutral particles, collision frequency is given by

$$
\vartheta_{\text {neutral }}=\bar{v} / \lambda
$$

For charged particles, the collision frequency, as derived by Lymberopoulos and Economou (1996), takes the form

$$
\vartheta_{\text {charged }}=\frac{|q|}{m \mu}
$$

where $q$ represents the amount of charge. It should be noted that equation above is valid when the conventional Ohm's law applies, where plasma conductivity is proportional to the electric field (Fridman \& Kennedy, 2004).

The kinetic theory relates the diffusion coefficient $D$ to mobility via the Einstein relation (Fridman \& Kennedy, 2004); for a charged particle represented by subscript par 


$$
D=\frac{k T_{p a r} \mu}{|q|}
$$

In non-thermal plasmas, electron diffusivity exceeds ion diffusivity by a few orders of magnitude due to much higher electron temperature and mobility.

\subsubsection{Free and ambipolar diffusion}

Generally, flux $F$ is obtained by summing advection and diffusion

$$
F= \pm \mu E n-D \nabla n \text {. }
$$

Number density of particles is represented by $n, E$ is the electric field vector, and $D$ is the diffusion coefficient. If electron and ion fluxes are independent of each other, plasma is under free diffusion. However, this is not the case in quasi-neutral plasma. Because the electron mobility is significantly higher, electrons are first to receive energy from the electric field. Electrons are depleted due to their higher flux, and the zone becomes positively charged. The quasi-neutrality restoring force creates a polarization field that decelerates the electrons and accelerates the positive ions (Fridman, 2008). The characteristic length of charge separation is given by the Debye length, which is proportional to electron temperature and inversely proportional to electron density

$$
\lambda_{D}=\sqrt{\frac{\varepsilon_{o} k T_{e}}{n_{e} e^{2}}}
$$

where $\varepsilon_{0}$ is the vacuum permittivity.

The characteristic length of plasma quasi-neutrality is given by the characteristic length of equivalent electron and ion flux (aka. ambipolar diffusion). The ambipolar diffusion coefficient, $D_{a}$, as derived by Fridman and Kennedy (2004) is given by 


$$
D_{a}=\frac{D_{i} \mu_{e}+D_{e} \mu_{i}}{\mu_{e}+\mu_{i}}
$$

\subsubsection{Degree of quasi-neutrality and boundary surface interaction}

The degree of plasma quasi-neutrality is obtained via its Debye length and plasma

frequency. The plasma frequency, $\omega_{p}$, represents the time-scale of the electron response to the electric field oscillations

$$
\omega_{p}=\sqrt{\frac{n_{e} e^{2}}{m_{e} \varepsilon_{o}}} .
$$

Derivations of Debye length and plasma frequency can be found in (Fridman and Kennedy, 2004) and (Lieberman \& Lichtenberg, 1994). Physically, an electron must have a velocity of $\lambda_{D} \cdot \omega_{p}$ to screen perturbations of the external electric field. This means that plasma with characteristic lengths much higher than the Debye length will maintain

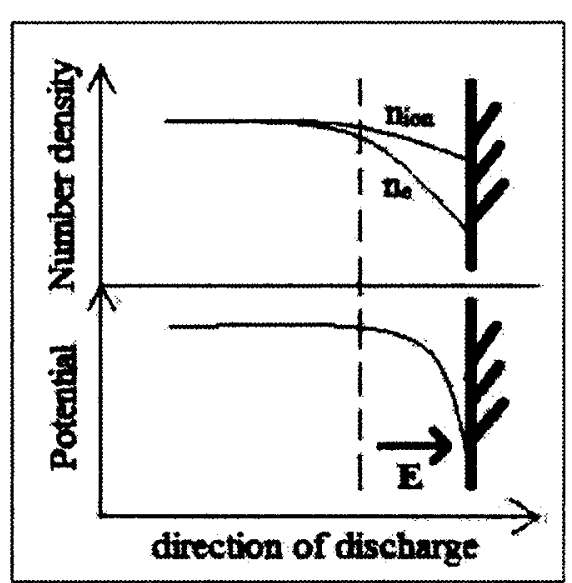

Figure 2.2: Plasma-wall interference quasi-neutrality. For the typical low pressure ( $\mathrm{mPa}$ to Pa range) glow discharges, the Debye length is on the order of $10^{-6} \mathrm{~m}$ and plasma frequency is in the $\mathrm{GHz}$ range (Fridman \& Kennedy, 2004). This means that quasi-neutral plasma is obtained for electrode separation much larger than $1 \mu \mathrm{m}$.

Plasma behavior near a solid surface (i.e. an electrode) boundary is critical. The restoring polarization field created due to the positive space charge is depicted in Figure 2.2. Since no quasineutrality can be reached at the plasma-wall interference, a plasma sheath is created. In order to sustain plasma, the sheath must accelerate the ions to a velocity equivalent to electron's thermal velocity, which is known as the Bohm velocity (Fridman \& Kennedy, 
2004). Positive ions striking the wall cause a secondary electron emission flux. This is given by

$$
\boldsymbol{F}_{\text {secondary }}=\gamma \boldsymbol{F}_{\text {ion }}
$$

where $\gamma$ is the secondary electron emission coefficient. The secondary emission electrons are accelerated into the plasma bulk, and ionization of these electrons is enough to sustain the plasma despite its low electric field. Secondary emission is essential to sustaining certain types of electric discharges. 


\section{Chapter: Thermodynamics and Kinetics in Plasma}

Plasma behavior in constrained discharges depends on the particle distributions, which are estimated from thermodynamics, and the potential field that is estimated from kinetics. This chapter provides an overview of statistical thermodynamics, elementary collisions, kinetics, and electromagnetics in plasma.

\subsection{Overview of Statistical Thermodynamics}

For systems with a very large number of molecules (for example, in atmospheric air, the number density of molecules is $10^{25} \mathrm{~m}^{-3}$ ), statistical methods must be used. These methods are built upon the concepts of quantum mechanics.

Recall that the energy of a molecule consists of a translational form and 3 internal forms (rotation, vibration, and electronic transition). Each of these forms of energy is discretized into allowed energy levels, and each level is composed of a number of energy states, which is the level's degeneracy. A fundamental key of quantum mechanics is that the energy state of a molecule is equally probable; thus given $Z$ number of states, the probability that any of the states exists at any given time is given by $Z^{l}$.

The probability of the energy level can only be determined if the particle distribution is known. This distribution is determined by the partition function, which defines the accessibility of an energy level based on its degeneracy and temperature. Note that the case of thermodynamic equilibrium mentioned earlier represents of a unique particle distribution, called the most probable particle distribution (MPPD) (Polak \& Lebedev, 1998). 


\subsection{The Internal Energies of Diatomic Molecules}

Polak \& Lebedev (1998) derive the statistical energy distributions for diatomic molecules under the following assumptions: atoms are rigid rotors and harmonic oscillators with fixed separation distance. Recall from the previous section, that temperature dictates the accessibility of the energy levels. In classical thermodynamics, this accessibility is determined by the heat capacity (which is the sensitivity of a form of energy to changes in temperature).

The heat capacity is given by

$$
c_{j}=\frac{d E_{j}}{d T_{j}}
$$

where subscript $j$ indicates any of the 4 forms of energy. As shown by Polak and Lebedev (1998), analysis of heat capacities reveals interesting peculiarities: $c_{\mathrm{rot}}$ and $\mathrm{c}_{\text {trans }}$ remain constant, whereas $c_{v i b}$ increases with temperature and $c_{\text {elec }}$ reaches a maximum. These peculiarities are very important when analyzing non-thermal plasma in air. Physically, the number of vibrationally excited molecules peaks at the maximum vibrational temperature, and the number of electronically excited states peaks for a specific electron temperature. Recall that the gas temperature is relatively insignificant in non-thermal plasma, and thus most of the energy in the system is due to electronic transitions (and also free electrons), and molecular vibration.

\subsection{Fundamental Collisions}

Reactions relevant to high pressure non-thermal discharges are discussed in this section. Emphasis will be made on the cross sections relevant to discharges in air. Details of chemical reactions relevant to plasma can be found in (Capitelli et al., 2000), (Fridman 
\& Kennedy, 2004) and (Roth, 1995). Particle collisions can be classified as either elastic or inelastic.

Consider the collision between an electron, $e$, and a neutral molecule, $A$,

$$
e\left(\varepsilon_{1}\right)+A \rightarrow e\left(\varepsilon_{2}\right)+A^{*}
$$

such that particle $A^{*}$ is formed. If the collision is elastic, the electron transfers kinetic energy $\left(\varepsilon_{2}<\varepsilon_{1}\right)$ to increase the kinetic energy of $A^{*}$ (gas heating). If the collision is inelastic, the electron transfers kinetic energy into internal energy of $A^{*}$, thus raising its rotational, vibrational and/or electronic state. Ionized, metastable, and dissociated species are formed this way. Inelastic collisions are very important in non-thermal discharges and are discussed next.

\subsubsection{Internal excitation}

Collisional excitation creates internally excited states. It should be noted that the electron-molecule interaction time in plasma is 2 orders of magnitude faster than molecular vibration or rotation (Fridman \& Kennedy, 2004). This means that the electronically excited states have 100 times shorter lifetimes than vibrational or rotational excited states, and atoms within molecule can be considered 'frozen' during the electronic transition. This is known as the Frank-Condon principle. Cross sections of excitation are determined semi-empirically; for instance, cross sections of $\mathrm{N}_{2}$ excitation to its first vibrational level range from $10^{-19}$ to $10^{-17} \mathrm{~cm}^{2}$ (MORGAN database, 2012).

\subsubsection{Direct impact ionization}

Direct impact ionization creates a positive ion, since the combined kinetic energy of the electron and particle exceeds the ionization potential of the neutral particle. Statistically, the ionization cross section is proportional to the natural logarithm of the electron energy (Fridman, 2008) 


$$
6_{\text {ionization }} \sim \frac{\ln \varepsilon}{\varepsilon}
$$

Electron impact ionization cross sections for $\mathrm{N}_{2}$ range from $10^{-19}$ to $10^{-15.5} \mathrm{~cm}^{2}$ (Fridman, 2008).

\subsubsection{Penning ionization}

Penning ionization also creates a positive ion; however, in this case, the total kinetic energy is provided the kinetic and internally excited states of the particles. For example, the collision of a He metastable can ionize $\mathrm{N}_{2}$, such that the Penning ionization cross section is on the order of $10^{-15} \mathrm{~cm}^{2}$ (Fridman, 2008).

\subsubsection{Photo-ionization}

Photo-ionization creates a positive ion due to the impact of a high energy photon with a neutral particle. Generally, the photon wavelength, in $\mathrm{m}$, required to ionize a molecule with ionization potential $I$, in $\mathrm{eV}$, can be obtained by (Fridman, 2008)

$$
\lambda<1.24 \cdot 10^{-6} / I
$$

For example, the photo-ionization cross section of $\mathrm{N}_{2}$ is approximately $2.6 \cdot 10^{-17} \mathrm{~cm}^{2}$ (Fridman, 2008), and requires a photon in the ultra-violet or x-rays spectrum (Lawrence Berkley National Laboratory, 2012).

\subsubsection{Electron attachment}

Electron attachment creates a negative ion. For example, attachment of an electron to an oxygen molecule is

$$
\mathrm{e}+\mathrm{O}_{2}+\mathrm{N}_{2} \rightarrow \mathrm{O}_{2}^{-}+\mathrm{N}_{2}
$$

The cross section of the reaction above is approximately $10^{-21}$ to $10^{-20} \mathrm{~cm}^{2}$ (Fridman, 2008). It should be noted that $N_{2}$ is a more effective $3^{\text {rd }}$ body than an electron due to the low degree of ionization in non-thermal plasmas. 


\subsubsection{Detachment}

In plasma discharges, electron detachment from negative ions that collide with internally excited particles or electrons is possible. For example, the reaction

$$
0^{-}+e \rightarrow O+2 e
$$

has cross section of approximately $3 \cdot 10^{-16} \mathrm{~cm}^{2}$ for electron energies of $3-10 \mathrm{eV}$ (Fridman \& Kennedy, 2004). For low temperature non-thermal plasma, a vibrational or electronic excited state of $\mathrm{O}_{2}$ is a more effective collision partner (Fridman,2008).

\subsubsection{Recombination}

For plasmas in molecular gases at high pressure, 3 body mechanisms of ion-ion recombination dominate. The rates of such reactions are recalculated to second order, based on the densities of positive and negative ions. For example,

$$
\mathrm{O}_{2}{ }^{-}+\mathrm{N}_{2}{ }^{+} \rightarrow \mathrm{O}_{2}+\mathrm{N}_{2}
$$

has a rate of $1.6 \cdot 10^{-7} \mathrm{~cm}^{3} / \mathrm{s}$. This rate is significantly higher than the rate of $\mathrm{N}_{2}$ ionization even though the activation energy is approximately equivalent to the ionization potential of $\mathrm{N}_{2}$ (Fridman, 2008).

\subsection{Kinetics Applicable to Plasma}

Particle distributions dictate transport properties and rates of chemical reactions. For plasma discharges, estimates of the energy distribution function and coupling to the potential field are of primary importance.

\subsubsection{Boltzmann kinetic equation}

Kinetics of individual plasma particles can be described via the Boltzmann kinetic equation, which is the total time derivative of the distribution function $f(r, v, t)$ 


$$
\frac{d f}{d t}=\frac{\partial f}{\partial t}+v \cdot \nabla_{r} f+a \cdot \nabla_{v} f
$$

In Equation 3.2, $f$ is a velocity distribution function (VDF) given by

$$
f=\frac{d N}{d r d v}
$$

where $d N$ is the number of particles within the volume $d r$, having velocities between $v$ and $v+d v$. Integration of the VDF over the velocity space gives the number density of particles (Lymberopoulos \& Economou, 1996),

$$
n(\boldsymbol{r})=\int_{0}^{\infty} f d v
$$

In Equation 3.2, $a$ is the local acceleration of a charged particle of mass $m$ and charge $q$, under the electromagnetic force field; this is given by

$$
a=\left[\frac{q(E+v \times B)}{m}\right]
$$

The numerator of Equation 3.5 is the Lorentz force due to the electric field $E$ and

magnetic field $B$. The total time-derivative of the distribution function, $\frac{d f}{d t}$, represents the temporal (or transient) effects of particle collisions. This is the most difficult term to estimate. The case of quasi-equilibrium is explained below.

Recall the quasi-equilibrium indicates the most probable state of a system; then $\frac{d f}{d t}=0$. The velocity distribution function obtained using the assumption above is called the Maxwellian or Maxwell-Boltzmann distribution. However, since the time-scale of particle collisions is inversely proportional to pressure, the total time derivative of the distribution function cannot be ignored in low pressure non-thermal plasma.

\subsubsection{Maxwellian VDF}

The Maxwellian VDF, illustrated in Figure 3.1 (adapted from Roth, 1995) is 


$$
f(v)=4 n / \sqrt[3]{\pi} \sqrt[3]{m / 2 k T} v^{2} e^{-m v^{2} / 2 k T}
$$

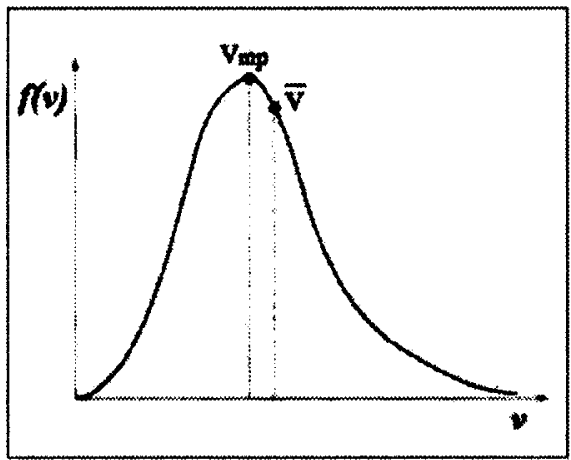

Figure 3.1: Maxwellian velocity distribution

where $T$ is the kinetic temperature, $m$ is the particle mass, and $n$ is number density.

It should be noted that the exponential term reflects on the conservation of kinetic energy, which is given by

$$
\varepsilon=\frac{1}{2} m v^{2}
$$

The most probable velocity $v_{m p}$, is the velocity at which VDF is maximum. The average velocity $\bar{v}$ is determined by integrating FDV along the velocity space, and dividing by the number density. For the Maxwellian FDV,

$$
\bar{v}=\sqrt{\frac{8 k T}{\pi m}}
$$

We will use $\bar{v}$ as the boundary condition for the thermal velocity in the $1 \mathrm{D}$ model.

\subsubsection{Maxwellian EDF}

A Maxwellian energy distribution function (EDF) can be obtained by plugging Equation 3.6 into Equation 3.7, which becomes

$$
f(\varepsilon)=2 N \sqrt{\varepsilon / \pi(k T)^{3}} e^{-\varepsilon / k T}
$$

In non-thermal plasma, it is common to determine the electron EDF (aka. EEDF). 
There are approximations of energy distribution functions for non-equilibrium cases, which can be found in (Fridman \& Kennedy, 2004). For example, Figure 3.2 (Fridman \&

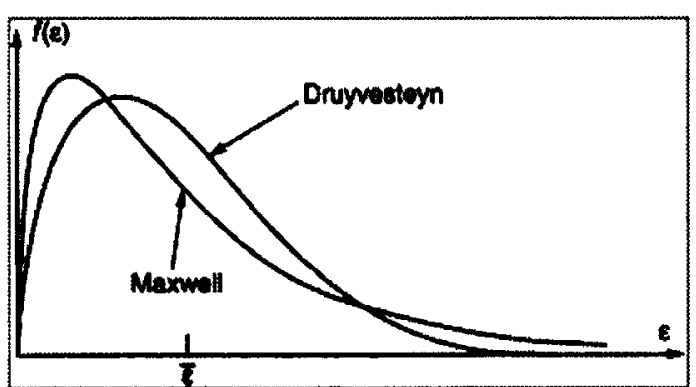

Figure 3.2: Maxwellian and Druyvesteyn energy distributions
Kennedy, 2004) illustrates the Maxwellian and Druyvesteyn EEDF distributions. The Druyvesteyn distribution is used for nonthermal plasma in atomic gases. It can be observed that the Druyvesteyn distribution has higher most probable electron energy. Also,

higher electron energy is more probable in the non-thermal distribution.

The mean electron energy $\vec{\varepsilon}$ is determined by integrating EEDF along the energy space, and dividing by the electron number density. It is given by

$$
\bar{\varepsilon}=\frac{3}{2} k T_{e}
$$

We will use Equation 3.10 to determine the mean electron energy in the 1D model.

\subsubsection{Transport and rate coefficients from the EDF}

Once the EDF is known, transport and rate coefficients can be obtained. For example, Kushner (2012) derives the electron impact reaction rate, which is given by

$$
k_{r}=\int_{0}^{\infty} 6(\varepsilon) f_{o}(\varepsilon) \sqrt{\varepsilon} d \varepsilon
$$

It should be noted that $f_{o}(\varepsilon)$ is the energy probability density (equivalent to the EEDF normalized by the number density), and $6(\varepsilon)$ are the collision cross sections. Similar equations can be derived for mobility, diffusion, and plasma conductivity. These equations can be found in (Farouk, 2009) and (Rutscher, 2008). 


\subsection{Importance of Statistics in Particle Reactions}

The classical Arrhenius equation gives the rate of a chemical reaction according to

$$
k_{r}=C e^{-E_{a} / R T_{\text {quast }}}
$$

where $C$ is an empirical constant, $E_{a}$ is the activation energy of the reaction, $R$ is the universal gas constant, and $T_{\text {quasi }}$ is a quasi-equilibrium temperature of reactants $\mathrm{A}$ and $\mathrm{B}$. Typically, this quasi-equilibrium temperature is the kinetic temperature; however, if high vibrational states exists such that $T_{v} \gg T_{g a s}$, then most of the reaction rate is due to the vibrational temperature (Fridman \& Kennedy, 2004).

Statistically, the reaction rate is determined by equations such as Equation 3.11. Unlike the Arrhenius equation, a statistically determined reaction rate does not assume a quasiequilibrium temperature; however, it requires an energy distribution of the reactants (kinetic or internal, depending on the type of reaction). Statistical estimation of reaction rates for non-equilibrium plasma states is mandatory. For most electric discharges, no quasi-equilibrium temperature exists among the energy degrees of freedom, and statistical methods are essential. The plasma model that will be developed in Chapter 7 is based on non-thermal EEDFs in air, and most of the transport and rate coefficients are derived statistically.

\subsection{Electromagnetics in Plasma Media}

This section presents an introduction to the electromagnetic field produced by moving charges within the plasma. Assumptions relevant to the electric discharge modeling are discussed. 


\subsubsection{Electromagnetic wave theory}

The electromagnetic field equations are given by the classical Maxwell equations. These can be manipulated, as in (Williams, 2012), to obtain the electromagnetic wave equations

$$
\begin{aligned}
& \frac{\partial^{2} E}{\partial^{2} t}=\varepsilon_{o} \mu_{o} \nabla^{2} E \\
& \frac{\partial^{2} H}{\partial^{2} t}=\varepsilon_{o} \mu_{o} \nabla^{2} H
\end{aligned}
$$

Equations $3.13 \mathrm{a}$ and $3.13 \mathrm{~b}$ relate the second time derivative of the electric and magnetic field to the Laplacian of the respective field; $\boldsymbol{E}$ and $\boldsymbol{H}$ are the electric and magnetic field intensities, and $\varepsilon_{o}$ and $\mu_{o}$ are the vacuum permittivity and permeability.

It should be noted that the electromagnetic wave equations above are valid for media where the relative permittivity constant, $\varepsilon_{\text {rel, }}$ is near unity (i.e. in air). The effect of a plasma medium is explained below.

\subsubsection{Electromagnetic propagation in plasma medium}

In a plasma medium, permittivity is a complex number given by

$$
\varepsilon_{c}=\varepsilon_{r e l}+i \varepsilon_{i} .
$$

The imaginary part, $\varepsilon_{i}$, accounts for plasma conductivity due to moving charges.

Conductivity is given by Ohm's law,

$$
\sigma=\frac{J_{\text {cond }}}{E}
$$

which is mostly due to plasma electrons,

$$
\sigma=\frac{n_{e} e^{2}}{m_{e} \vartheta_{e n}}
$$

where $n_{e}$ is the electron number density, $e$ is the elementary charge, $m_{e}$ is the electron mass, and $\vartheta_{e n}$ is the electron-neutral collision frequency. 
Fridman \& Kennedy (2004) show that the solution to the complex permittivity given by Equation 3.14 is

$$
\varepsilon_{c}=\left(1-\frac{\omega_{p}^{2}}{\omega^{2}+\vartheta_{e n}^{2}}\right)+i \frac{\sigma}{\omega \varepsilon_{o}} .
$$

The real part of permittivity specifies the strength of the polarization field in plasma, depending on the electromagnetic wave frequency $\omega$, plasma frequency $\omega_{p}$, and the electron-neutral collision frequency $\vartheta_{e n}$.

\subsubsection{Oscillating electromagnetic fields}

Consider the electromagnetic wave equations, Equations 3.13a and 3.13b. Plugging Equation 3.17 into Equation 3.13a, one obtains the electric field in a plasma medium

$$
\nabla^{2} E-\varepsilon_{o} \mu_{o} \ddot{E}-\mu_{o} \sigma \dot{E}=0 .
$$

In steady state, the electric field has an attenuation factor, $\kappa$, which describes its exponential decrease of intensity as it propagates through a dielectric. It can be shown that

$$
E(r)=E_{o} e^{i(\omega t-\kappa r)}
$$

satisfies Equation 3.18. Physically, the electromagnetic wave is attenuated by $1 / e$ when the penetration depth is $\operatorname{Re}(1 / \kappa)$. This penetration of electromagnetic energy through a dielectric is known as the skin depth (Fridman \& Kennedy, 2004) and is given by

$$
\delta=\sqrt{\frac{2}{\omega \mu_{o} \sigma}} .
$$

Skin depth is a measure of electrical conduction distance. Equation 3.20 indicates that skin depth is infinite for DC current $(\omega=0)$. This means that electrical conduction occurs through the entire length of the dielectric medium. As the driving frequency increases, the skin depth decreases. Consider an electric discharge where the 
characteristic length is the electrode gap, $d$. If $\delta \ll d$ then external fields and currents are only significant within the skin depth boundary layer (Fridman \& Kennedy, 2004).

\subsubsection{Assumptions relevant to modeling discharges}

The sub-sections below validate the electrostatic assumption that is typically used for modeling electric discharges. The validity of this assumption to modeling SPDI is discussed in Chapter 6.

\subsubsection{The electrostatic approximation}

The Lorenz force, which appears in the Boltzmann kinetic equation, describes the electromagnetic force on a charged particle with velocity $v$,

$$
F=q(E+v \times B)
$$

The magnetic force can be ignored for $\mathrm{AC}$ discharges where the electrode gap is insignificantly small compared to the skin depth, $d \ll \delta$.This is equivalent to saying that conductivity occurs on a length-scale much larger than the gap size, and hence the electrostatic approximation is valid.

\subsubsection{Coupling the charge field to the electrostatic field}

In the electrostatic approximation, the time rate of change of the magnetic flux is neglected; namely, the electric field is irrotational. This allows the electric field to be approximated by the gradient of the potential field

$$
E=-\nabla V
$$

In the electrostatic case, Poisson equation is valid, and given by

$$
\nabla \cdot\left(\varepsilon_{o} \varepsilon_{r e l} \nabla V\right)=-\rho .
$$


In Equation 3.23, $\rho$ is the space charge density, which is given by

$$
\rho=\sum_{z} q_{z} n_{z}-e n_{e}
$$

where $z$ is the number of ionic species, $q_{z}$ is the charge of each ionic species, and $n_{z}$ is the number density of each ionic species. For the case of single charge ions, $q= \pm e$, the elementary charge. 


\section{Chapter: Overview of the Electric Discharge and its Modeling}

In this chapter, the physics of electrical breakdown and the glow discharge is presented. For theories of other electric discharges, the interested reader should consult Fridman (2008) or Roth (1995). Subsequently, hydrodynamic modeling of the electric discharge is presented; such simulations are discussed in Chapter 5 and presented in Chapter 9.

\subsection{The Electric Discharge}

An electric discharge is a flow of plasma between a pair of electrodes with a potential difference. There are 2 important aspects of an electric discharge: breakdown, and sustainability.

\subsubsection{Breakdown}

Breakdown is a transient process of nearly instantaneous resistance decrease of the plasma medium, where a highly conductive and ionized channel forms. This occurs when the ionization mean free path is much less than the electrode gap size, and is determined by the breakdown voltage (Fridman, 2008). The similarity parameter $P \cdot d$ (pressure multiplied by the gap distance) determines the type of breakdown.

\subsubsection{Sustainability}

Sustainability indicates the maintenance of a specific type of plasma, which requires energy flow equilibrium. Specifically, the electrical power input must balance the conduction/radiation heat losses, energy diffusion, and rates of energy exchange due to collisional processes. If the energy flow is not at equilibrium, the electric discharge is prone to transition and/or extinction. 


\subsection{General DC Discharges}

The general voltage-current characteristics of DC discharges for "parallel plate"

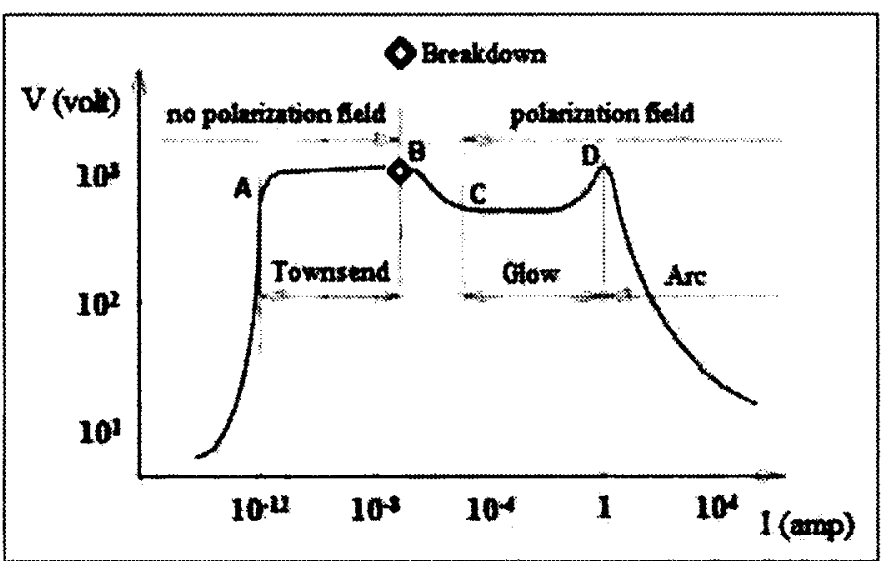

Figure 4.1: Characteristics of general DC discharges electrodes in atmospheric air are shown in Figure 4.1 (adapted from

Smith et al., 2011). Initially, discharge current forms due to background ionization and radiation (i.e. free electron density in atmospheric air is around $10^{10}$ support ionization and charges recombine. The transition to self-sustained discharge occurs at point $\mathrm{A}$ in Figure 4.1.

\subsubsection{Electrical breakdown}

Breakdown, or sparking, is a transient process where resistance decreases once a critical voltage is reached. This phenomenon occurs when the dielectric media becomes conductive. Plasma flow is constricted within the conductive channel, which has a radius of approximately $200 \mu \mathrm{m}$ in atmospheric pressure air (Smith et. al, 2011). There are two fundamental theories of breakdown: Townsend and streamer (Fridman, 2008).

Townsend breakdown is a gas ionization process initiated by free electrons near the cathode that have enough energy to cause a growing electron flux toward the anode. Similarly, the slower ion flux drifts toward the cathode. Breakdown is achieved when the 
positive ions reach the cathode, and an electron is emitted from the cathode surface (Fridman \& Kennedy, 2004). Let

$$
(\alpha-\beta) d=\mathrm{G}
$$

where $\alpha$ and $\beta$ coefficients represent the number of ionizations and electron attachments per unit distance respectively, $d$ is gap distance, and $\mathrm{G}$ is variable representing the condition for breakdown. It can be shown (Fridman \& Kennedy, 2004) that for breakdown according to Townsend theory,

$$
G=\ln \left(\gamma^{-1}+1\right)
$$

where $\gamma$ is the secondary electron emission coefficient described in Chapter 2.

Coefficients $\alpha, \beta$ and $\gamma$ are determined semi-empirically, and depend upon the type of gas and the reduced electric field.

The transient period leading to Townsend breakdown is characterized by insignificant electric field distortion due to the space charge (no polarization field). However, experiments indicate that breakdown occurs much faster than the time required for the positive ions to drift to the cathode, when similarity parameter $P \cdot d$ exceeds a threshold (i.e. $P \cdot d>4000$ Torr $\mathrm{cm}$ according to Fridman $\&$ Kennedy, 2004). This mechanism of

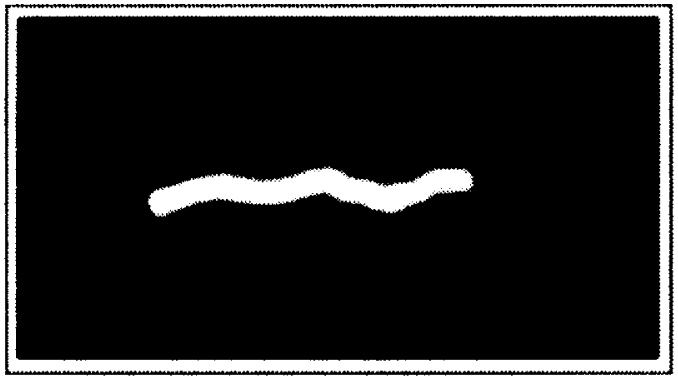
breakdown is known as streamer breakdown, or sparking.

Figure 4.2 (Wagner \& Brandenburg, 2011)

shows a photograph of a spark discharge.

Figure 4.2: Photograph of a spark discharge Collisional de-excitations that occur around the ionized channel produce a high luminosity discharge. 
The transition from Townsend to sparking occurs due to a significant polarization field that increases the total field around the "ionization avalanche". The overall ionization is accelerated because secondary avalanches can now propagate along the positive space charge left by slower ionic charges (Fridman, 2008). As a result, highly conductive localized channels called streamers form, which require photo-ionization to be sustained. The streamer velocity is orders of magnitude higher than the ionization velocity during Townsend breakdown. Fridman \& Kennedy (2004) show that streamer breakdown is given by Meek's condition

$$
G \geq 20 \text {. }
$$

Streamers can be cathode-directed if the primary electron avalanche reaches the anode, or anode-directed if streamers initiate prior to reaching the anode and propagation is toward both electrodes (Fridman \& Kennedy, 2004). A spark forms when the streamer(s) connect the electrodes for a long enough period of time to breakdown the media. It should be noted that sparking can develop into an arc if the discharge voltage is not reduced immediately after breakdown (see Figure 4.1).

\subsubsection{The glow discharge}

A glow discharge is a steady-state plasma that is sustained by the generation-loss balance of plasma particles. It is the most common source of low temperature non-thermal plasma. The glow discharge is characterized by a significant polarization field that restructures the total electric field and causes a voltage drop (section B-C in Figure 4.1). 


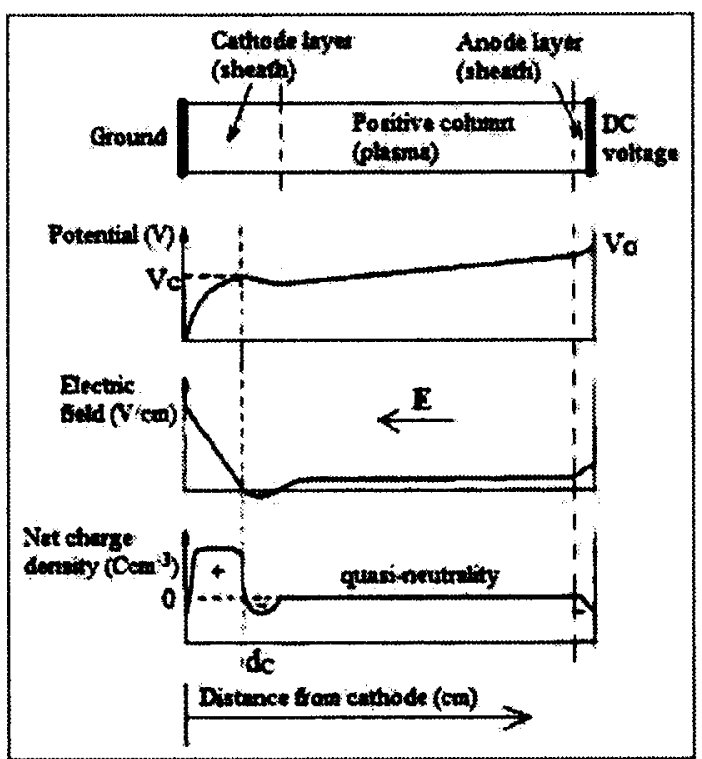

Figure 4.3: Physics of the DC glow discharge

The physics of the DC glow discharge is shown in Figure 4.3 (adapted from Roth, 1995). The discharge is composed of the cathode layer, positive column, and the anode layer. These regions are explained below sequentially from the cathode to the anode, and illustrated in Figure 4.4 (adapted from Wagner \& Brandenburg, 2011). In the cathode layer, a sheath is formed due to electron depletion (see sheath theory in Chapter 2). The positive space charge produces a restoring positive electric field gradient (the electrostatic field gradient is given by Equations 3.23). Electrons repelled from the cathode surface via secondary emission

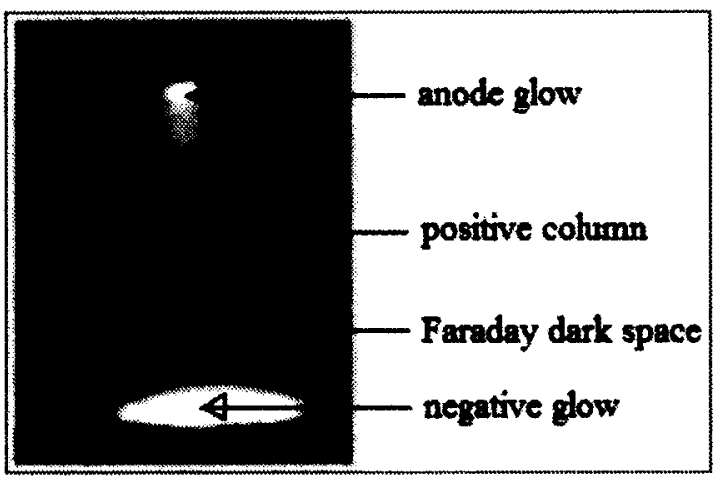

Figure 4.4: Photograph of a glow discharge accelerate in this field gradient, and excite vibrational and electronic levels of the gas. This forms the cathode glow (Fridman, 2008). Adjacent to the cathode glow is the cathode dark space, which forms due to ionization (according to Equation 3.11, the ionization rate increases when the population of high energy electrons increases). It should be noted that the cathode glow and cathode dark space are not identifiable in Figure 4.4 due to the intensity of the negative glow that follows the cathode dark space. The negative glow is the brightest region since electron energy cannot cause further ionization, but favors collisional excitation (rate of excitation is similar to Equation 3.11, but cross-sections better match the electron energy). The cathode layer ends with a 
transition to the Faraday dark space, where electron recombination and diffusion enhance the electric field to establish the positive column. Ambipolar diffusion (see Chapter 2) enforces quasi-neutrality in this region, which sustains the discharge in very low electric fields (order of $1 \mathrm{~V} / \mathrm{cm}$ ); electron density is generally $10^{15}-10^{16} \mathrm{~m}^{-3}$ and its kinetic energy is around $1-2 \mathrm{eV}$ (Fridman, 2008) for glow discharges in air. Low uniform glow is observed along the positive column. A negatively charged sheath (the anode layer) forms next to the positive column due to positive ion depletion. The electric field strength increases in the anode layer, and then transitions into the anode glow.

The cathode layer is the most important region of the glow discharge, because sufficient voltage drop must occur to sustain the plasma. The cathode potential drop, $V_{C}$, and the length of the cathode region, $d_{C}$, can be estimated from Roth (1995) or Fridman $\&$ Kennedy (2004). For parallel plate atmospheric pressure glow in air,

$$
\begin{gathered}
V_{C} \approx 375 \mathrm{~V} \\
d_{C} \approx 6.6 \cdot 10^{-4} \mathrm{~cm}
\end{gathered}
$$

The glow discharge transitions into an arc discharge at point $D$ in Figure 4.1, where the ion energy exceeds the work function of the cathode material (Fridman, 2008). The work function is the minimum energy required to remove an electron from the cathode surface; if this occurs, the secondary electron emission coefficient, $\gamma$ (see Chapter 2) becomes 2 to 3 orders of magnitude larger.

\subsection{Discharge Transitions}

Discharge instabilities develop when the current density exceeds the threshold of the particular regime. For example, in Figure 4.1, the glow-to-arc transition occurs when the 
current exceeds $1 \mathrm{~A}$. A transition of the electric discharge is actually a thermal instability. Recall that the steady-state discharge is sustained by the generation-loss balance of charged particles. Similarly, gas heating must be balanced by the excitation-relaxation of kinetic energy. For non-thermal plasma, such as the glow discharge, the channels of instability are explained below.

In a non-thermal plasma in air, most of the electron energy is transferred to the internal energy states of the $\mathrm{N}_{2}$ and $\mathrm{O}_{2}$ molecules; specifically, excitation of the vibrational temperature, $T_{v}$, is dominant. Vibrational energy is converted into translational energy through a relaxation process. The degree of vibrational-translational (V-T) exchange dictates the amount of gas heating. The local increase in gas temperature, $T_{\text {gass }}$ causes a decrease of gas density, $\mathrm{N}_{\mathrm{gas}}$, which enhances the reduced electric field. This promotes further ionization (recall that electrons are first to receive energy from the electric field) and increases the local electron density, $\mathrm{n}_{\mathrm{e}}$. This process is equivalent to a positive feedback system, which is inherently unstable.

\subsection{Special Cases of Electric Discharges}

Two important aspects relevant to the SPDI discharge are the high pressure medium, and the electric field non-uniformity due to the spark plug geometry.

\subsubsection{Discharge in high pressure (atmospheric and above)}

The degree of ionization is inversely proportional to pressure squared, $n_{e} / N_{g a s} \propto 1 / P^{2}$ (Fridman \& Kennedy, 2004). This means that steady state non-thermal discharges are easier to sustain in low pressures. For example, experimental observations of SPDI in 
Chapter 6 illustrate a pressure threshold for sustaining a steady mode of discharge. On the other hand, electron collision frequency is directly proportional to pressure (Fridman \& Kennedy, 2004), to first order approximation. This means that collisional intensities increase, and the electron population can reach a close to quasi-equilibrium distribution (see Chapter 3).

\subsubsection{Highly non-uniform electric field}

Electric discharges with geometries such as point-plate can have stability fields (sum of external and space charge fields) several times less than the critical field required for breakdown. This means that sparking does not necessarily occur when the first streamer connects the electrodes (Naidis, 1999). For example, oscilloscope data of SPDI discharges indicate voltages that are an order of magnitude higher than the parallel-plate case shown in Figure 4.1.

\subsection{Hydrodynamic Modeling of Plasma}

The Boltzmann kinetic equation (given by Equation 3.2) can be solved via the continuum approach if the distribution function $f$ is known. Moments of the Boltzmann kinetic equation provide the conservation equations, which are derived in Balcon (2007). Mass continuity is obtained by integrating Equation 3.2 over the velocity space

$$
\frac{\partial n}{\partial t}+\nabla \cdot(n v)=\sum_{\text {coll } t} R_{i}
$$

where $n$ is the number density, $v$ is velocity, and $R_{i}$ is the net rate of species production due to collision coll $i$.

Conservation of momentum is obtained by multiplying Equation 3.2 by $m v$ then integrating over the velocity space 


$$
\begin{aligned}
\frac{\partial}{\partial t}(n m v)+\nabla & (n m v \cdot v) \\
& =-\nabla(n k T)+n q(E+v \times B)-n m v v_{m}
\end{aligned}
$$

In Equation 4.6, the LHS is the sum of the local acceleration and inertia, where $m$ is the particle's mass. The RHS represents sources of momentum due to: the pressure gradient $P=n k T$ ( $T$ is the kinetic temperature), the momentum exchange with the electromagnetic field ( $q$ is charge, $E$ is the electric field, $B$ is the magnetic field), and the momentum exchange via elastic collisions $\left(\vartheta_{m}\right.$ is the effective momentum exchange frequency).

If the local acceleration and inertia are ignored, momentum reduces to the drift-diffusion approximation (Lymberopoulos \& Economou, 1996). This approximation is frequently used in plasma simulations. The drift-diffusion flux $F$ is given by

$$
\boldsymbol{F}=n v=q n(\mu E)-D \nabla n
$$

The mobility in given by $\mu=\frac{q}{m \vartheta_{m}}$ and the diffusion coefficient is given by $D=\frac{k T}{m \vartheta_{m}}$ Conservation of energy is obtained by multiplying Equation 3.2 by $m v \cdot v$ then integrating over the velocity space

$$
\begin{aligned}
\frac{\partial\left(n E_{\text {tot }}\right)}{\partial t}+\nabla & \left(n v E_{\text {tot }}\right) \\
& =-\nabla(n k T) v+n q(E+v \times B) \cdot v+\nabla \cdot K \nabla T \\
& -\sum_{\text {collt } t} E_{\text {tot_i }}
\end{aligned}
$$

The LHS of Equation 4.8 is the sum of total energy time-rate of change, and energy convection. The RHS is the sum of the pressure work, energy exchange with the electromagnetic field, conduction ( $K$ is the thermal conductivity), and collisional energy exchange. For non-thermal plasmas, both elastic and inelastic collisions must be 
considered. It should be noted that the total energy term $E_{\text {tot }}$ contains thermal and kinetic energy gained from the drift in the electric field. However, most simulations assume that the kinetic energy is insignificant compared to the thermal energy (Lymberopoulos \& Economou, 1996).

\subsubsection{Local field approximation and its limitations}

The easiest way to obtain the transport (i.e. mobility, diffusivity) and collision rate coefficients is to assume that these coefficients scale with the local reduced electric field $E / N$. This requires that the particle mean free path is much smaller than the spatial variations of the electric field (Balcon, 2007). However, in the plasma bulk, electron energy exchange with the electric field and collisional processes cannot be considered a local process due to ambipolar diffusion. To account for non-local effects, it is common to solve the energy equation for electrons, then obtain transport and rate coefficients known from the local field (S. Pancheshnyi, personal communication, 2012). This is explained below.

Let the electron mean energy be represented by the electron temperature $T_{e}$, and the transport and rate coefficients represented by a function $€$. Then,

$$
\begin{gathered}
\because €=\epsilon\left(\frac{E}{N}\right) \text { and } T_{e}=T_{e}\left(\frac{E}{N}\right) \\
\therefore €=€\left(T_{e}\right)
\end{gathered}
$$

As illustrated above, by solving the mean electron energy, electron transport and rate coefficients can be obtained in the non-local field approximation. 


\subsubsection{EEDF in non-thermal plasma}

Most electric discharges have non-equilibrium energy distributions. Hence adequate modeling of these discharges requires estimates of electron transport and rate coefficients based on the local mean electron energy. These coefficients can be obtained for equilibrium and non-equilibrium distributions from LXcat (MORGAN database, 2012), which is an open-access website that uses Bolsig $+^{3}$ (Hagelaar \& Pitchford, 2005).

For an equilibrium electron distribution, the EEDF is Maxwellian, and LXcat requires a range of electron temperatures and fixed gas temperature. For non-equilibrium distributions, the range of local reduced electric fields and gas temperature must be known. Then Bolsig + computes the EEDF in order to determine the electron transport and rate coefficients (for example, the rate of ionization is determined by Equation 3.11).

\footnotetext{
${ }^{3}$ Bolsig+ is a solver of the Boltzmann kinetic equation in low ionized and low temperature plasma
} 


\section{Chapter: Literature Review}

This chapter is divided into 2 parts. The first part summarizes literature relevant to plasma modeling using the hydrodynamic approach. The second part provides an overview of electric discharge imaging and published experimental data of combustion ignition via non-thermal plasma.

\subsection{Plasma Modeling}

The central problem in plasma modeling of electric discharges is the numerical simulation of spatial and temporal variations in the electric field and number density of plasma particles.

\subsubsection{Simulations that solve the electron and ion transport}

One and two dimensional (axisymmetric) models of micro-gap breakdown in atmospheric air are presented by Smith et al. (2011). Although mobility is assumed constant, and ion diffusivity is ignored, the models identify the Townsend regime of

Figure 4.1 accurately. The authors determine the 1 and 2D model breakdown potential coincide for gaps larger than $100 \mu \mathrm{m}$. Another model based on the local field approximation is developed by Koh and Park (2003), to simulate pulsed DC corona in atmospheric air. Corona is a non-thermal discharge that occurs prior to breakdown. Nanosecond rise-time pulses were used to prevent sparking. A positive space charge ionization wave is observed to propagate due to the electric field increase ahead of ionization front. The authors determine that positive ions and electrons play key roles in the dynamics of the ionization front. Similarly to Koh and Park, Naidis (1999) presents positive streamer propagation in pulsed DC corona. Production of $\mathrm{O}$ and $\mathrm{N}$ radicals is determined along the rising electric field, using electron impact and stepwise dissociation 
rates of $\mathrm{O}_{2}$ and $\mathrm{N}_{2}$. Simulations show that electric field strength is an order of magnitude higher at the streamer head than at its channel. Subsequently, Naidis (1999) presents kinetic processes responsible for streamer-to-spark transitions on the nanosecond timescale, which are verified with experimental data. The sparking transition is observed by rapid vibrational-translational relaxation (i.e. gas heating). The author determines that accumulation of $\mathrm{O}, \mathrm{N}$, and $\mathrm{NO}$ radicals at transition has the most influential impact on the electron energy balance.

\subsubsection{Simulations that solve the electron, ion and electron energy transport}

Models that solve 3 moments of the Boltzmann kinetic equation usually simulate discharges in a quasi-steady state. Farouk (2009) models DC and RF micro glow discharges in atmospheric pressure argon, helium, hydrogen, and methane. Simulations of the DC glow illustrate the anode and cathode sheaths, and the positive column as presented by the theory in Chapter 3. The RF glow simulations in argon are of particular interest regarding SPDI, as they illustrate voltage-current waveforms that correspond to SPDI's glow mode that will be presented in Chapter 6. Farouk's model simulates the 2 known modes of glow discharge, $\alpha$ and $\gamma$, and captures the $\alpha-\gamma$ transition. Simulated peak temperature in the $\gamma$ mode is $280 \mathrm{~K}$ higher than the $\alpha$ mode, whereas sheath thickness is reduced by a factor of 5. Similarly, Balcon (2007) presents a numerical model of atmospheric pressure RF glow discharge in argon. Simulations illustrate the breakdownto-glow transition, where conduction currents surpass displacement currents. Axial profiles of electron energy in the $\gamma$ mode show temperatures four times higher near the electrode, which are due to the rising electric field in the sheath; these values agree with Farouk's model. 
Similar simulations of non-thermal atmospheric pressure discharges are presented by Hagelaar (2008), and Shi and Kong (2005). A common feature of quasi-steady state discharge models is the semi- or fully-implicit coupling to the electric force field, and flux boundary conditions (BCs) for the species. Indirect coupling to the electrostatic force field does not have a constrained time-step, and this reduces simulation times significantly. While no wall diffusion BCs are used in (Shi \& Kong, 2005), most models use wall velocities composed of thermal and drift (Farouk, 2009), (Balcon, 2007), and also diffusion (Hagelaar, 2000).

\subsection{Electric Discharge Imaging}

Although numerical models agree with theory of electric discharge, experimental methods of capturing the spatial and time propagation of electric discharge are of equal importance. Charge-coupled device (CCD) cameras are generally used, but imageintensified (ICCD) cameras with time resolution of $0.1 \mathrm{~ns}$ are required to capture

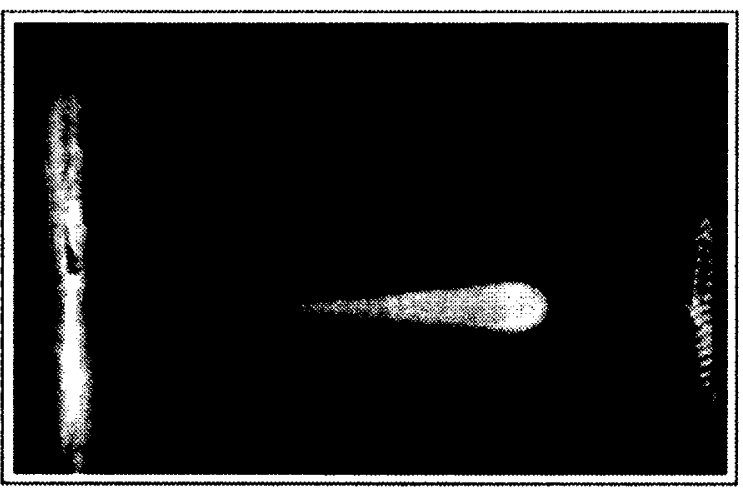

Figure 5.1: The first electron avalanche observed streamer propagation. Van Veldhuizen \&

Rutgers (2001) present a chronological summary of electric discharge diagnosis in atmospheric gases.

The first image of an electron avalanche is shown in Figure 5.1 (Van Veldhuizen \& Rutgers, 2001). This was observed by

Raether via light scattering, which provides no time resolution. The "avalanche growth" between parallel electrodes in $\mathrm{N}_{2}$ (cathode on the left) verifies the Townsend theory. 
Recall that the avalanche to streamer transition requires electric field enhancement due to the space charge. To understand the dynamics and the breakdown/sparking transition, time resolution is necessary.

Streamer imaging can be obtained via image-intensified cameras, or Schlieren photography, which is commonly used in combustion flame imaging. Schlieren

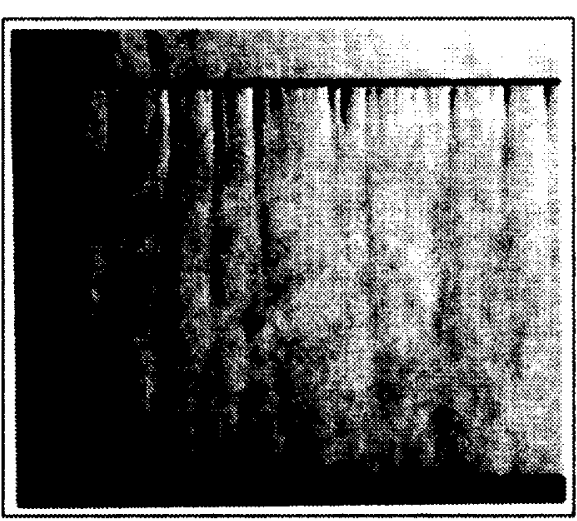

Figure 5.2: Schlieren photograph of streamer discharges photographs are sensitive to the refractive index of the medium. Figure 5.2 (Van Veldhuizen \& Rutgers, 2001) illustrates a Schileren photograph of the streamer path in a wire-plane corona discharge after $100 \mu$ s. It should be noted that no significant gas heating was observed prior. This makes sense since streamers initially affect the vibrational and

electronic excited states of molecules (recall the Frank-Condon principle in Chapter 3). Collisional de-excitation into the translational states produces gas heating, and these require larger time-scales.

\subsection{Combustion via Plasma Ignition}

Combustion via plasma ignition is a very active topic that raises much interest lately. Recent experiment data indicate that the type of ignition has significant effects on the flame kinetics and overall combustion efficiency. It is well-known that combustion is a chain reaction mechanism of 3 main stages: initiation, branching, and termination, where the rates of branching reactions are most influential on the flame propagation velocity. Most of these reactions involve radicals that are formed via dissociation of the $\mathrm{O}_{2}$ and $\mathrm{N}_{2}$ 
molecules; therefore higher concentrations of such radicals can accelerate flame propagation.

The fundamental difference between thermal and non-thermal ignition is the mode of ionization. In thermal plasma, the electron-gas collisions cause significant translational and rotational excitation. Then hydrocarbons are dissociated thermally due to elevated gas temperatures. In non-thermal plasma, the electron-gas collisions raise the vibrational and electronic energies of the molecules, although on a much smaller time-scale. The electron-gas translational energy exchange is weak (Lieberman \& Lichtenberg, 1994); hence hydrocarbon dissociation is mostly due to collisions of the internally activated energy states.

\subsubsection{The non-thermal plasma advantage}

Over the past decade a number of combustion experiments were performed with ignition via non-thermal plasma. The focus of these experiments was to maintain non-equilibrium via a dielectric barrier or high pulsed frequency to prevent the transition into an arc. By preventing local heating of the gas mixture, higher concentrations of electronic and vibrational excited states were produced. Consequently, production of radicals was observed, which enhanced the combustion flame velocity and sustained leaner mixtures (Starikovskii, 2005), (Rosocha et al., 2004). The lean mixture is characterized by an airto-fuel ratio (AFR) lower than the stoichiometric AFR. The advantage of lean mixtures is the reduction of the combustion temperature, which is beneficial for NOx reduction. Bellenoue et al. (2005) present several advantages of corona discharge (or non-thermal discharge for the matter) over the conventional spark discharge. For instance, rates of 
ionization by electron impact are higher due to a more favorable non-thermal EEDF; according to Equation 3.11, the rate of ionization increases if a larger part of the electron distribution has enough kinetic energy required for the ionization collision; see Figure 3.2 for an example. Also, radiation losses are reduced due to lower gas temperature, which means that more discharge energy contributes to flame sustainability. A key component of streamer discharges is multi-site ignitions in a larger volume, rather than localized heating of the arc discharge.

\subsubsection{Relevant combustion experiments involving non-thermal plasma}

Starikovskii (2005) investigates oxidation of various hydrocarbons (methane to hexane), where ignition is a $60 \mathrm{~mJ}$ nanosecond pulsed discharge. The experiments illustrate high emission spectra of $\mathrm{H}, \mathrm{OH}$ and $\mathrm{CH}$ radicals, which indicates acceleration of the branching reactions. The author also observes that the non-thermal ignition can reduced combustion temperatures by as much as $600 \mathrm{~K}$. Combustion is spatially uniform at $0.5 \mathrm{~atm}$, and the number of ignition sites increase at $2 \mathrm{~atm}$. A separate experiment was conducted on a

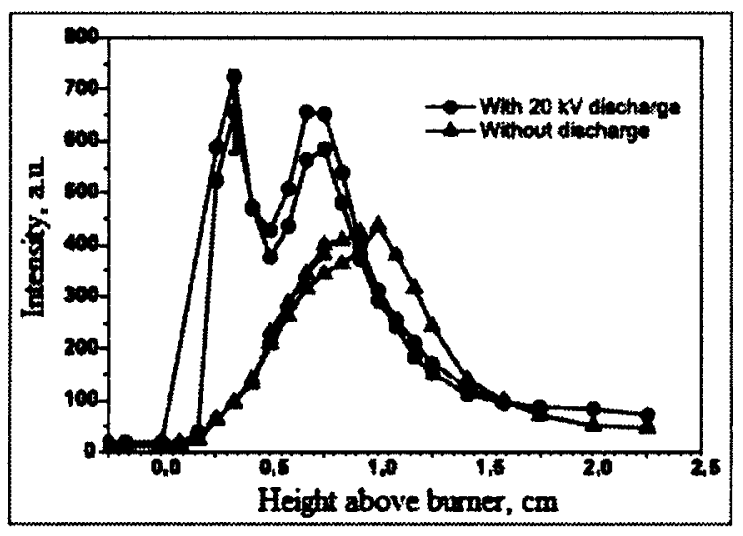

Figure 5.3: $\mathrm{OH}$ radical production under nonthermal plasma propane-air flame, where the ignition source was an $8 \mathrm{~mJ} 20 \mathrm{kV}$ AC dielectric barrier discharge (DBD). The positive polarity DBD doubled the flame propagation velocity for equivalence ratios, $\Phi^{4}$, ranging from 0.65 to 0.75 . Starikovskii determined that cathode directed streamers

\footnotetext{
${ }^{4} \Phi$ is the ratio of the stoichiometric AFR to the actual AFR, and characterizes the richness of the mixture
} 
caused the highest flame acceleration. Figure 5.3 (Starikovskii, 2005) shows the emission spectrometry of the $20 \mathrm{kV}$ DBD discharge. The second intensity peak indicates production of the $\mathrm{OH}$ radical under DBD. Starikovskii speculates that flame propagation is controlled by the concentration of radicals that cause higher chemical energy release during $\mathrm{O}_{2}$ dissociation.

Singleton et al. (2010) compare atmospheric pressure ethylene-air combustion with ignition via conventional spark or transient pulsed streamers. Their experiments indicate ignition delays of $10.9 \mathrm{~ms}$ for spark, 5.5 and $6.1 \mathrm{~ms}$ for the low and high energy streamers respectively. Temperature measurements show that after $100 \mathrm{~ns}$, streamer

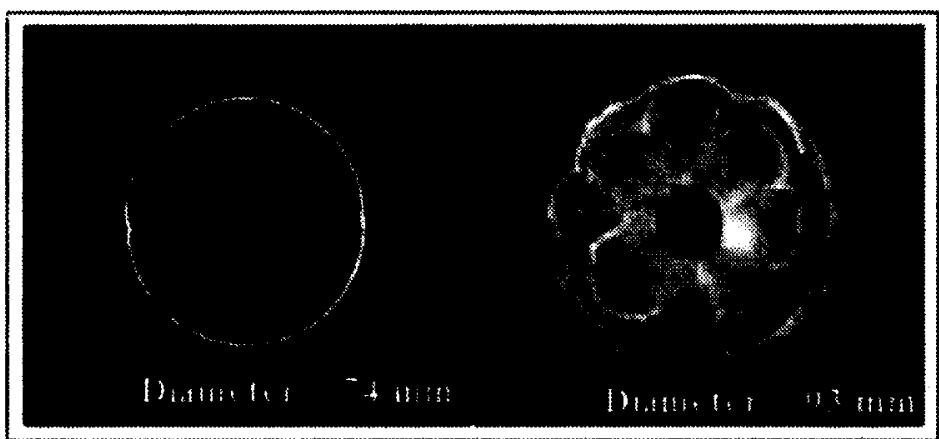
Figure 5.4: Turbulent fame with non-thermal plasma ignition development $6 \mathrm{~ms}$ after the temperature remains at $300 \mathrm{~K}$, whereas temperature in the spark channel reaches $4000 \mathrm{~K}$.

Figure 5.4 (Singleton et al., 2010) illustrates the flame

initial flame kernels. As Figure 5.4 illustrates, transient streamers can enhance combustion propagation due to multi-site ignition and enhanced turbulence. These observations confirm Starikovskii's hypothesis indirectly: high energy electrons produced in the streamer head are elementary to the production of radicals that intensify combustion (Starikovskii, 2005).

Liu et al. (2004) investigate the effects of discharge energy and electrode geometry on the methane-air flame ignited by conventional-spark and nanosecond pulsed corona. They 
determine that pulsed corona ignitions require an order of magnitude higher minimum energy than the spark. However, for $75 \mathrm{~mJ}$ discharge energy, ignition delay is reduced 20

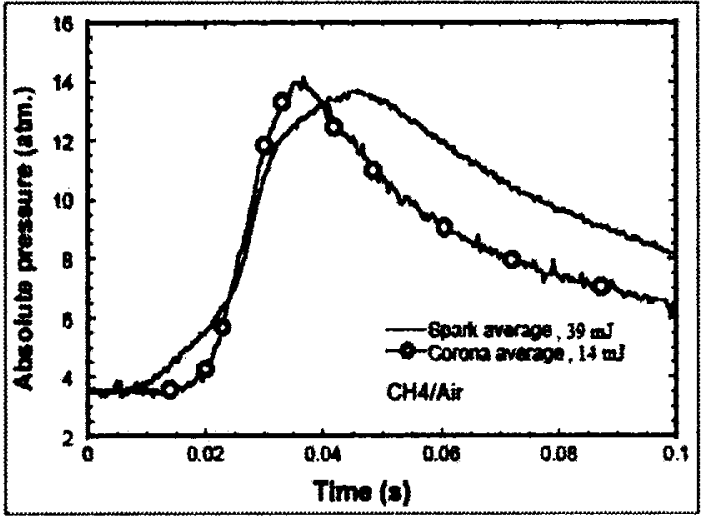

Figure 5.5: Combustion pressure history for spark and corona ignition to $30 \%$ for lean mixtures ignited by corona.

Combustion pressure rise time was additionally reduced by the multi-pin corona (190 mJ); multi-pin spark plugs have multiple powered electrodes. This indicates that multisite ignition reduces the flame burning times, similarly to the findings of Singleton et al.

Figure 5.5 (adapted from Liu et al., 2004) shows the combustion pressure history of single-pin spark and corona ignitions for stoichiometric $\mathrm{CH}_{4}$-air. Combustion rise time is reduced by a factor of 2 for corona ignition. Furthermore, calculations indicate $60 \%$ energy efficiency for corona, and only $5 \%$ for spark. The authors attribute reduced ignition delay and higher energy efficiency due to a chemical mechanism.

Rosocha et al. (2004) investigated the effects of dielectric barrier discharge on the

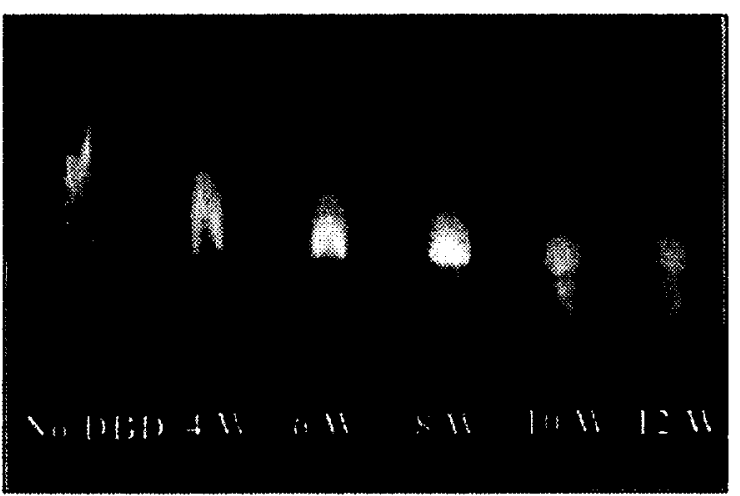

Figure 5.6: Flame propagation under DBD discharge propane-air flame velocities and blowout limits. The blowout limit is characterized by the lowest equivalence ratio that sustains the flame. In their experiments, non-thermal plasma pre-excites the fuel prior to mixing. With DBD pre-excitation, combustion is sustained for $30 \%$ lower equivalence ratios.

However, higher blowout limits are observed only when the discharge energy density 
exceeds a threshold. Mass spectrometry illustrates higher $\mathrm{H}_{2} \mathrm{O}, \mathrm{CO}_{2}$, and lower hydrocarbon concentrations with DBD pre-excitation, which is direct confirmation of more complete combustion. The photographs in Figure 5.6 (Rosocha et al., 2004) illustrate the enhanced flame speed in the presence of DBD. The first image on the left in Figure 5.6 has no DBD pre-excitation, whereas the following images, in order from left to right, represent DBD pre-excitation of $4,8,6$ and $10 \mathrm{~W}$ respectively. At $6 \mathrm{~W}$ and above, flame propagates in the opposite direction as well, which indicates higher reactions rates and flame stability.

The corona and DBD ignitions used in the previously presented experiments have pulse periods on the nanosecond time-scale. The non-thermal character of these ignitions was shown to benefit combustion processes by creating more active species. However, SPDI plasma has a periodic pulse of $28 \mu \mathrm{s}$ and its duration can be extended to the ms timescale. The hypothesis of SPDI (which will be discussed in Chapter 6) is that the nonthermal plasma support phase can accelerate the flame kernel propagation. The work by Bellenoue et al. (2005) verifies such a hypothesis by studying stoichiometric and lean methane-air flames ignited by either an automotive spark of $100 \mu$ s duration, or $5 \mathrm{~ms}$ and $20 \mathrm{~ms}$ positive-polarity corona. The corona is generated using single and 4-pin electrode configurations. Discharge imaging shows that the 4-pin configuration produces larger discharge volume and higher discharge power for equivalent gaps and voltages. However, for comparison purposes, the 1-pin electrode configuration is used.

The authors produce Schlieren photographs of the spark and corona ignited methane-air flames, which are shown in Figure 5.7a (adapted from Bellenoue et al., 2005). The 
corona ignited combustion indicates obvious flame acceleration, and specifically, the longer pulse intensifies gas turbulence.

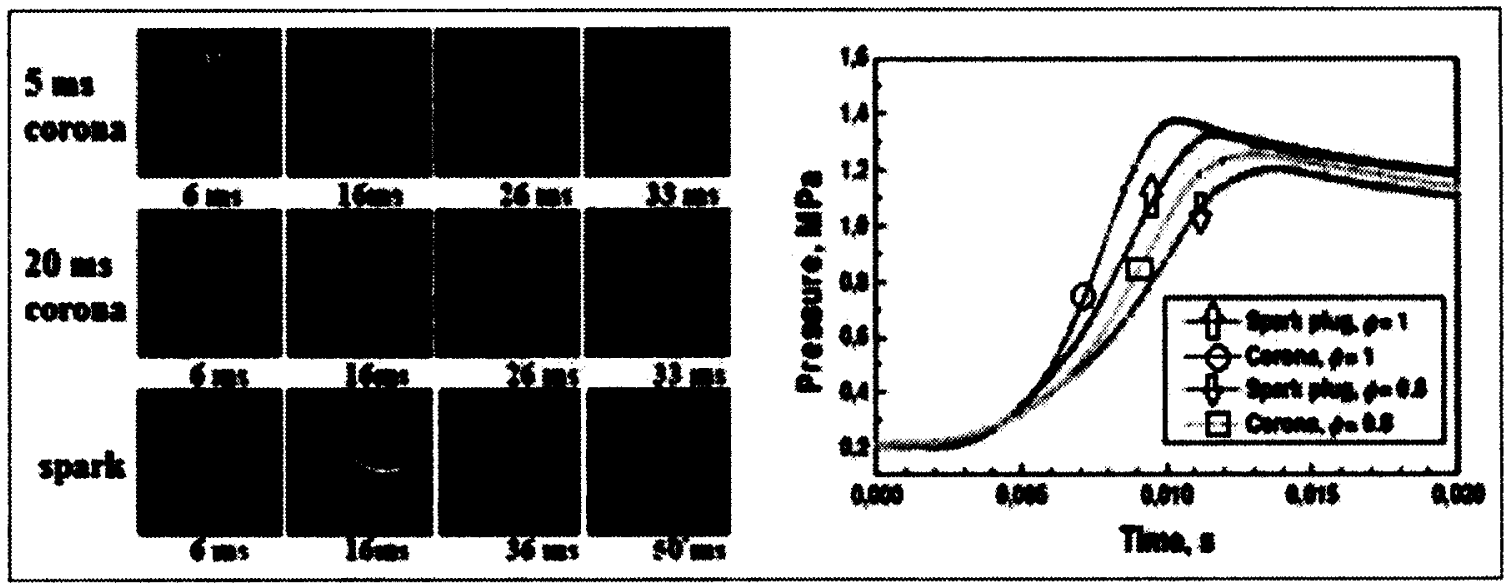

Figure 5.7: a) Schlieren protographs of the flame propagation, and b) Pressure combustion history of spark and corona ignition

Comparison between the spark and $20 \mathrm{~ms}$ corona ignition shows $25 \%$ (for stoichiometric mixtures) and $54 \%$ (for $\Phi=0.8$ ) faster combustion, and higher rate of pressure rise as shown in Figure 5.7b (adapted from Bellenoue et al., 2005). The authors hypothesize that the rate of pressure rise is due to chemically active species, and the benefit of corona ignition is due to larger spatial distribution of radicals. 


\section{Chapter: Experimental Observations of SPDI}

This chapter contains two parts. In the first part, oscilloscope data of SPDI discharges in air and SPDI assisted combustion in the experiments by Giordano (2012) are analyzed. It should be noted that with delayed setup, SPDI extended as much as $30^{\circ}$ after top-deadcenter (TDC), whereas timing for conventional spark ignition is around $20^{\circ}$ before TDC (Giordano, 2012). In the second part of this chapter, photographs of the SPDI discharge are compared to atmospheric pressure discharges in the literature. Then, the assumptions relevant to modeling SPDI are validated, and a hypothesis of SPDI assisted combustion is presented.

\subsection{The Experimental Setup}

The experimental setup is outlined in Figure 6.1 below. More details can be found in (Giordano, 2012). The dotted box in Figure 6.1 illustrates the focus of this research:

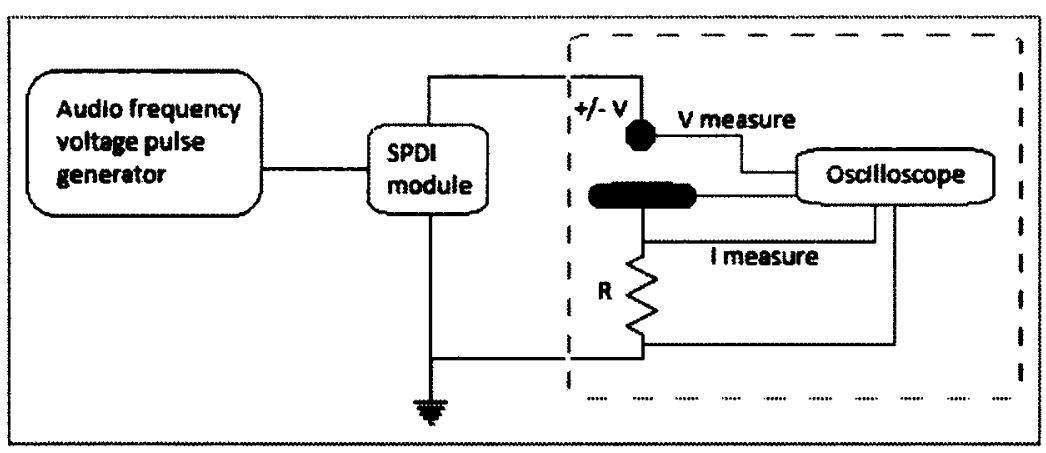

Figure 6.1: Overview of the experimental setup for testing SPDI NOTE the highly non-uniform electrode geometry namely, the analysis is

based on the discharge

voltage and current

measured by the oscilloscope at $1 \mu \mathrm{s}$ intervals.

\subsection{The Plasma Support Phase}

Recall that the plasma power is given by the duty cycle of the circuit transistor. However, it should be noted that the actual power is greatly influenced by the conditions of the medium, mainly the gas pressure, temperature and turbulence. For the interface software 
in SPDI, the duty cycle of the circuit transistor represents plasma power relative to a discharge in ambient air and a $7 \mathrm{~mm}$ gap.

\subsection{SPDI Supported Combustion}

For the combustion experiments, SPDI duration and engine loads were varied and oscilloscope data were obtained (Giordano, 2012). Note that only oscilloscope data representative of a power setting of $44 \mathrm{~W}$ are presented below.

\subsubsection{Plasma support phase under low/high load and duration}

The plasma support phase of one discharge event under low engine load is shown in Figure 6.2; low and high duration are illustrated for comparison. At low duration, the voltage and current waveforms do not stabilize, but are in phase; hence the power is absorbed by the gas. At high duration, the voltage magnitude increases by a factor of 2 compared to low duration. Furthermore, the voltage-current waveforms indicate a significant phase shift where current leads voltage (the net impedance is capacitive). At around $2.5 \cdot 10^{4} \mu \mathrm{s}$, the high duration discharge in Figure 6.2 illustrates a significant DC current bias. 


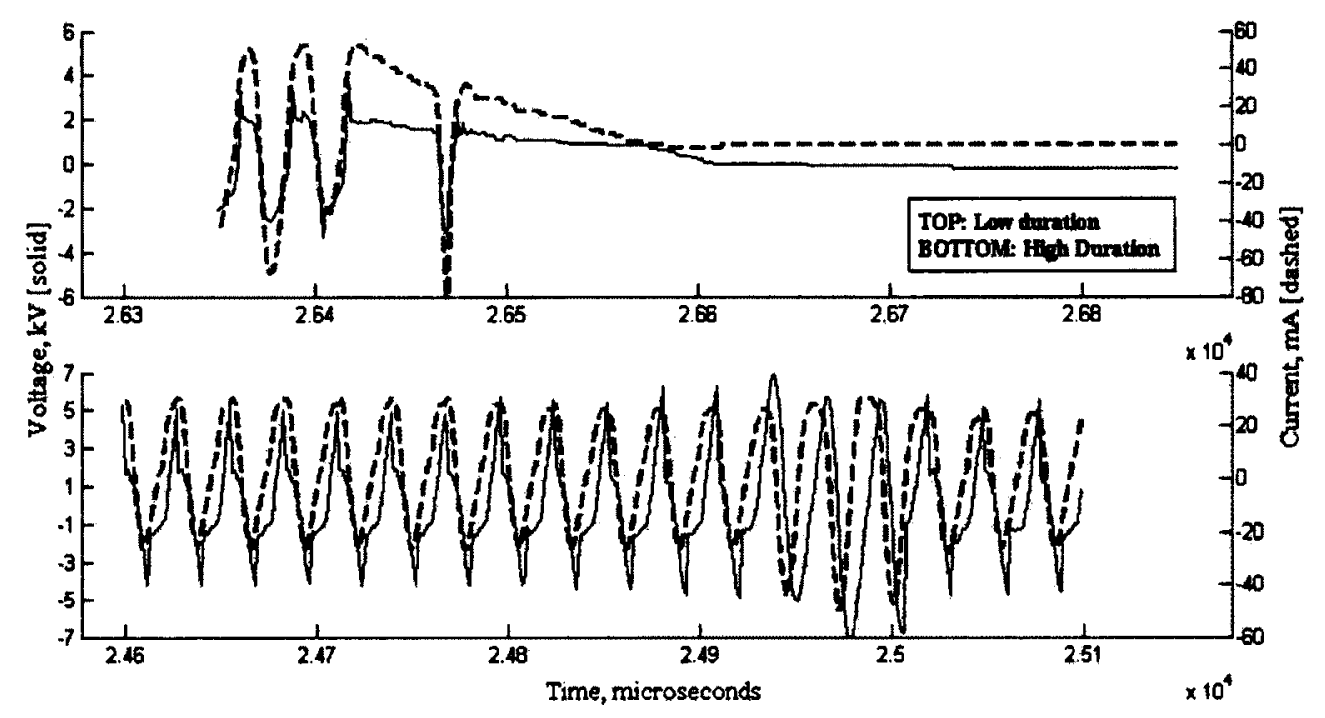

Figure 6.2: Oscilloscope data of SPDI ignition at low engine load NOTE: the last pulse on the TOP figure is the coil discharge (around $2.645 \cdot 10^{4} \mu \mathrm{s}$ )

It should be noted that a significant phase shift between the voltage and current indicates that a substantial amount of energy is reflected (aka. reactive power). Reactive power is a parasitic form of power in the circuit due to the inductive/capacitive nature of plasma.

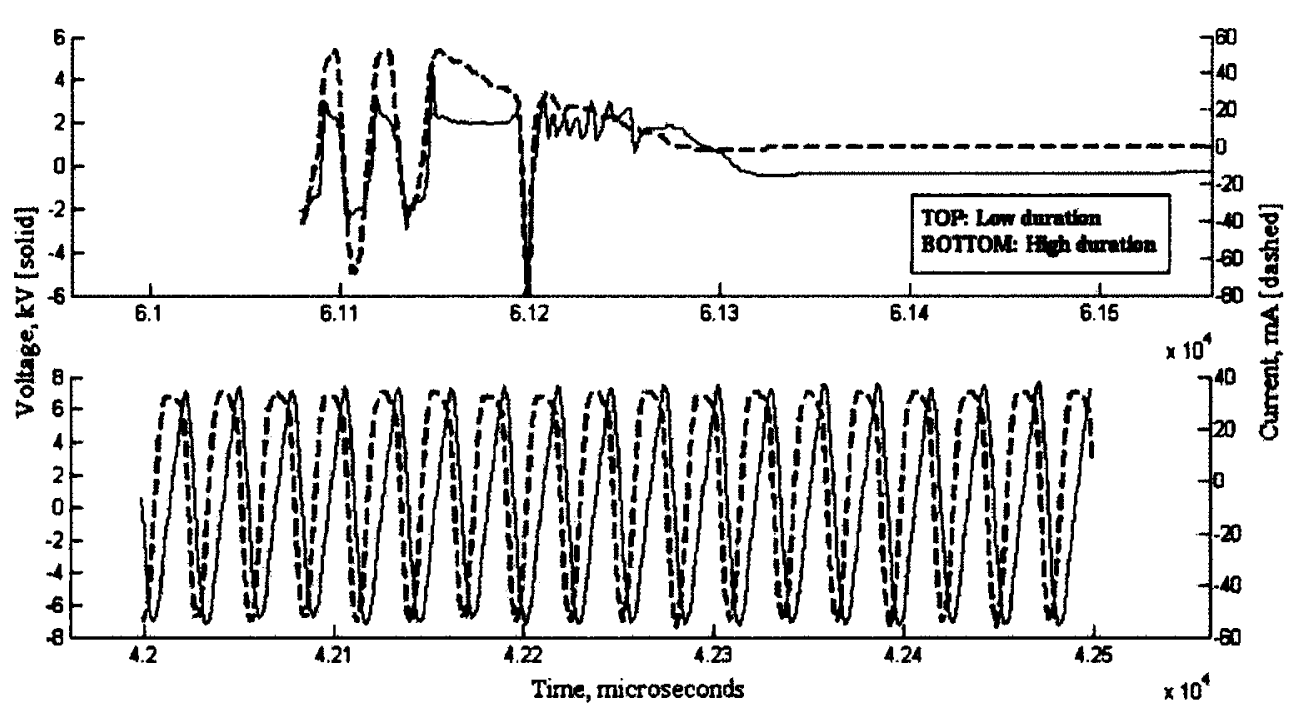

Figure 6.3: Oscilloscope data of SPDI ignition at high engine load NOTE: the last pulse on the TOP figure is the coil discharge (around 6.12.10 $\mu \mathrm{s}$ ) 
The plasma support phase of one discharge event under high engine load is shown in Figure 6.3; again, low and high duration are displayed. Voltage-current waveforms of low duration are similar to those at low duration and low engine load (see Figure 6.2). However, for high duration and high engine load, the discharge is characterized by a significant DC current bias and net capacitive phase-shift. It should be noted that this mode of discharge is sustained at the relatively higher pressures that are expected for higher load conditions. A comparison of Figures 6.2 and 6.3 is summarized below:

- At low duration, most of the discharge power is absorbed by the gas. From Figures 6.2 and 6.3, it can be seen that voltage and current are in phase.

- At high duration, there is a significant amount of reflected power in the circuit and a large DC bias current exists; this means that discharge kinetics are affected by the electrode's polarity. From Figures 6.2 and 6.3, it can be seen that voltage and current are out of phase, and the negative current amplitude is higher than the positive current amplitude.

- Pressure and gas composition changes during combustion affect the plasma. From Figures 6.2 and 6.3, it can be seen that the transition from low to high duration plasma support creates a phase-shift.

\subsection{SPDI in Pressurized Air}

SPDI was also tested in air at ambient temperature and pressures that varied from atmospheric to 150 psi gauge, at 10 psi intervals. Eight discharge events were recorded for each pressure. An air medium removes the effects of gas composition change during combustion and reduces problem complexity. The voltage-current waveforms during the plasma support phase in air reveal 2 discharge modes that resemble the waveforms for 
combustion ignition at low duration and high duration shown previously, as we will show next. Again, oscilloscope data representative of a power setting of $44 \mathrm{~W}$ is presented.

\subsubsection{The 2 discharge modes}

A sample of the first discharge mode is shown in Figure 6.4. This mode is observed for discharges in air from atmospheric to $100 \mathrm{psi}$ gauge pressure.

The voltage oscillations near the negative voltage peaks are due to the transient changes in the gap resistance. However, most of the discharge energy is absorbed by the gas.

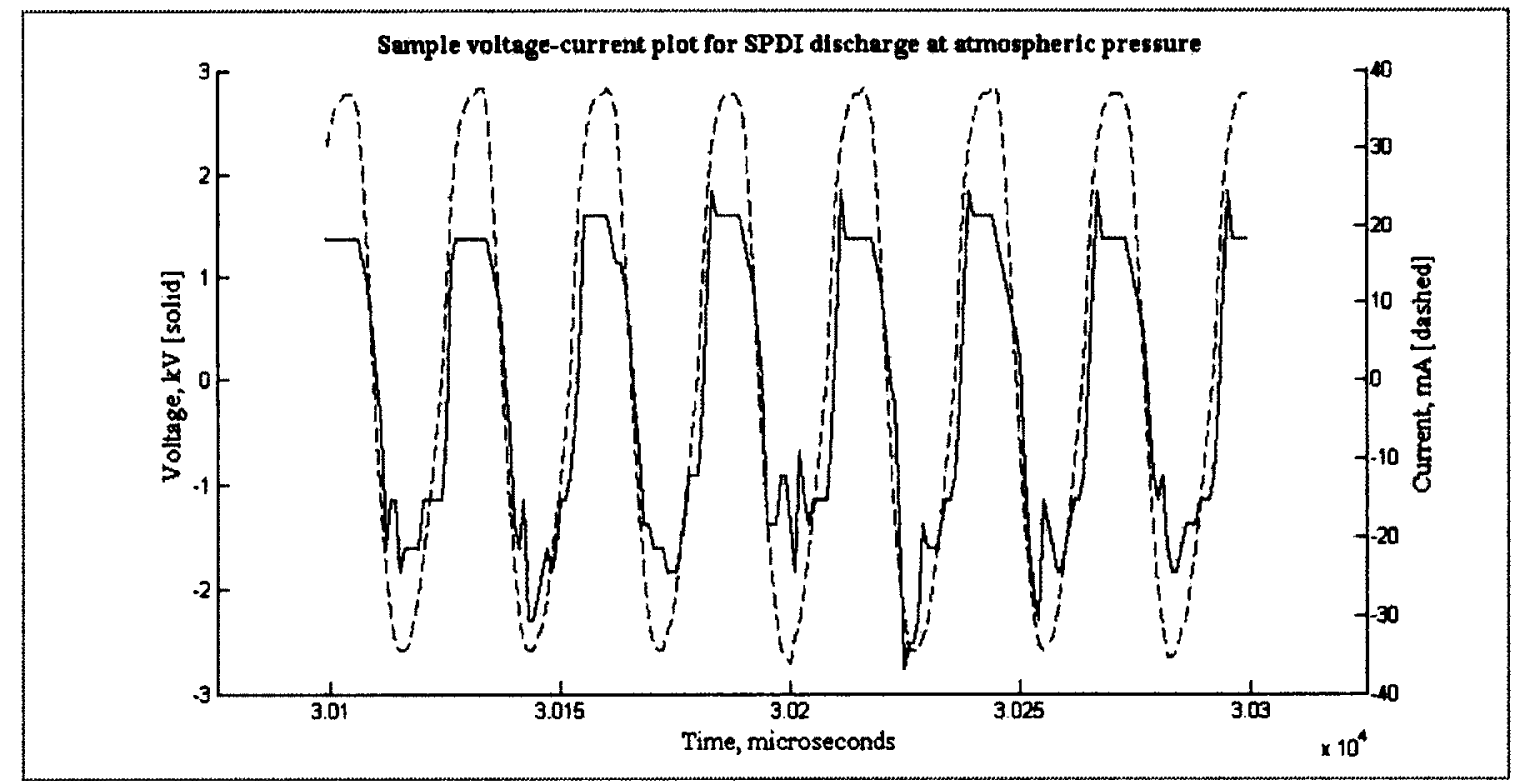

Figure 6.4: Oscilloscope data of the first mode of discharge in air at atmospheric pressure

A sample of the second discharge mode is shown in Figure 6.5. This mode is observed for SPDI discharges in air from 120 psi gauge to 150 psi gauge pressure. In comparison to the first mode, a very large phase shift exists, meaning that most discharge energy is reflected. The voltage amplitude is increased by a factor of 4 compared to the voltage amplitude of Figure 6.4, and significant DC current bias of approximately $-30 \mathrm{~mA}$ exists. 
The phase-shift and DC current bias are the main characteristics of the second mode of discharge in air.

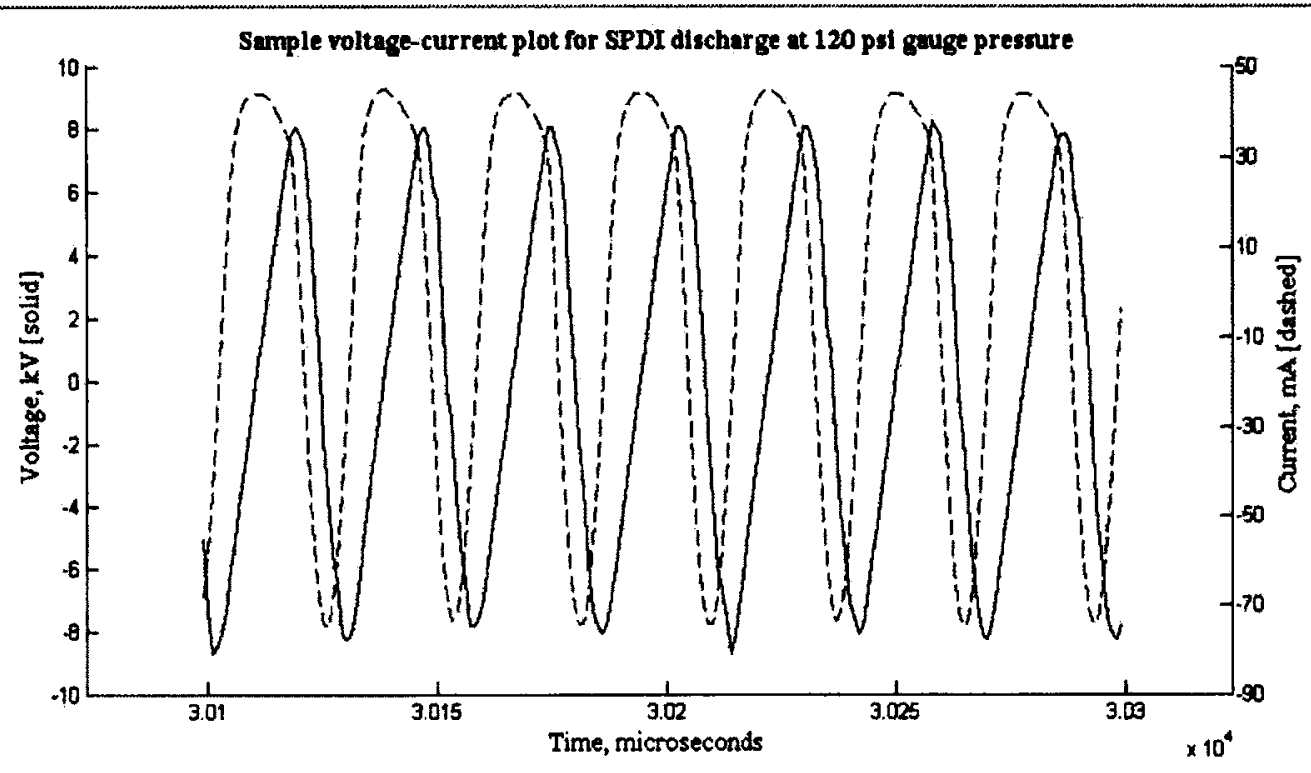

Figure 6.5: Oscilloscope data of the second mode of discharge in air at 120 psi gauge pressure

\subsubsection{Discharge imaging}

Giordano (2012) captured the breakdown and plasma support phase of the SPDI

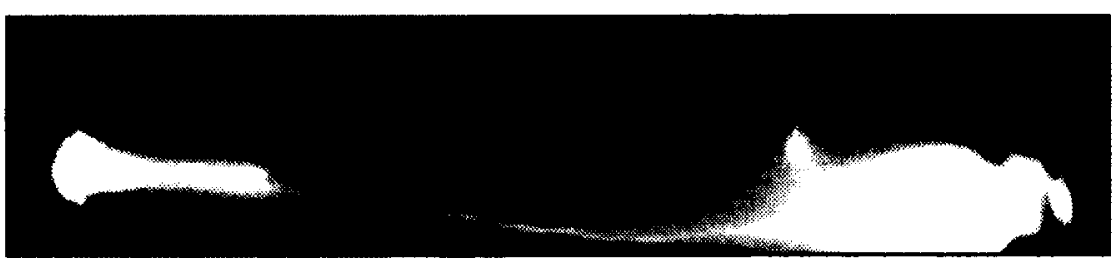

Figure 6.6: Sample image of the breakdown produced by SPDI discharge in air via a

CCD camera. The

breakdown glow intensity increases

with pressure, which indicates that the similarity parameter $P \cdot d$ is proportional to breakdown intensity. The sample image in Figure 6.6 (Giordano, 2012) illustrates the spark produced by SPDI at breakdown.

For SPDI discharges in air between 0 and $110 \mathrm{psi}$ gauge pressure, the plasma support phase glows at the electrode-air gap interfaces, as illustrated in Figure 6.7 (Giordano, 
2012). This mode of discharge resembles the typical DC glow discharge, hence the kinetics are heavily based on the glow discharge theory presented in Chapter 4 . The voltage-current waveforms in Figure 6.4 indicate a steady-state glow discharge.

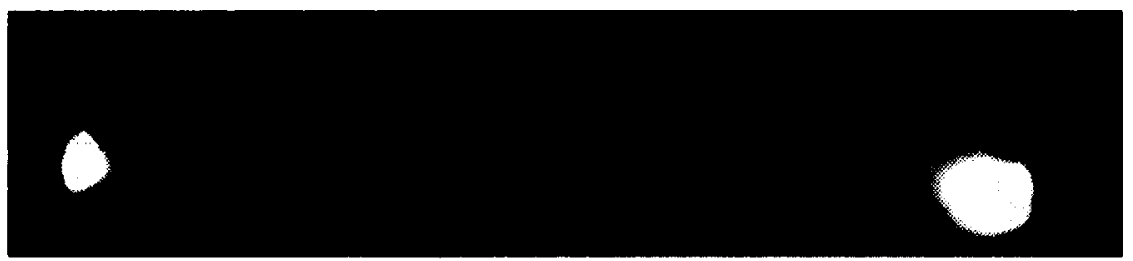

The plasma support phase cannot be captured by the

Figure 6.7: Sample image of the glow discharge produced by SPDI CCD camera when the air pressure exceeds 110 psi gauge (Giordano, 2012). Hence for a power setting of 44 $\mathrm{W}$, this pressure is the main factor of thermal instability, or precisely, the transition between the 2 discharge modes.

It should be noted that $\mathrm{CCD}$ cameras do not have enough time resolution to capture streamer propagation. Most probably, the high electrode potential and large $P \cdot d$ parameter stimulate streamer discharges. Recall from Chapter 4 that streamer formation occurs when the polarization field reaches the external (applied) electric field. An important observation that supports the streamer discharge theory is the oscilloscope data in Figure 6.5, which indicate 50\% higher negative current compared to the positive current. This indicates that the SPDI discharge directed by the anode is more intense that the discharge directed by the cathode; it should be noted that the anode directed streamer forms faster in comparison to the cathode directed streamer (see Chapter 4). Then the DC bias current observed in the second discharge mode can be explained by the discharge polarity. If SPDI creates streamer discharges at high pressure, then an ICCD camera or Schlieren photography is required to capture the discharge propagation. 


\subsection{Discharge Power Analysis}

The discharge power of the plasma support phase is determined by computing the area bounded by the voltage-current hysteresis curve; this approach was used by Azooz and Talal (2011). The Matlab solver by Owaid (2011) was used to determine the area bounded by the hysteresis curve.

In Figure 6.8, the average discharge power of 50 plasma support pulses is calculated for all 8 ignition events, for a range of pressures (error bars shows 1 standard deviation for the 8 ignition events). The average power is approximately $52 \mathrm{~W}$, which is $18 \%$ higher than the power setting. This difference can be attributed to reducing the gap size from 7 $\mathrm{mm}$ to $1 \mathrm{~mm}$ (recall that the power setting in SPDI is relative to a $7 \mathrm{~mm}$ gap size).

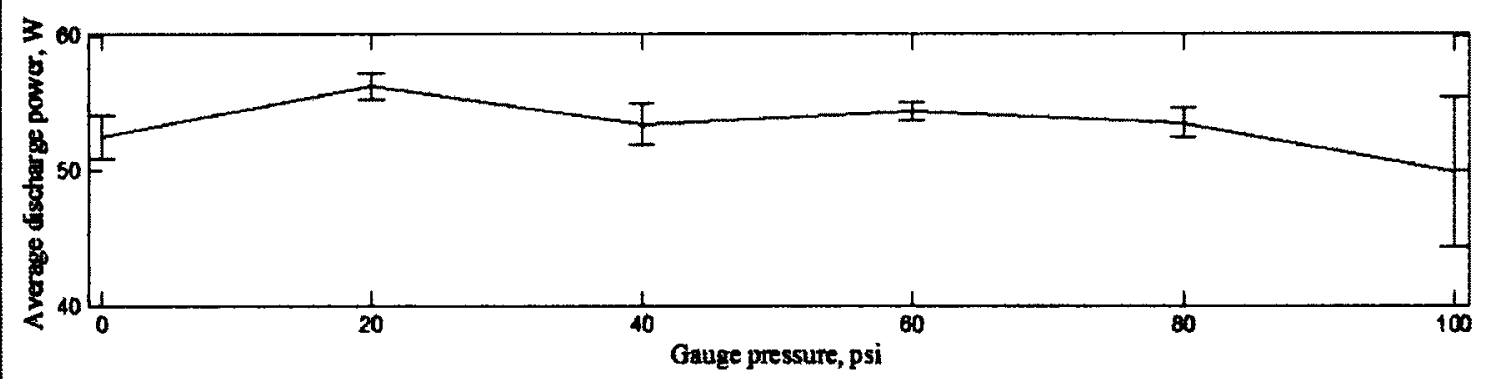

Figure 6.8: Average discharge power in the first discharge mode for the pressures tested Error bars show 1 standard deviation for the 8 ignition events

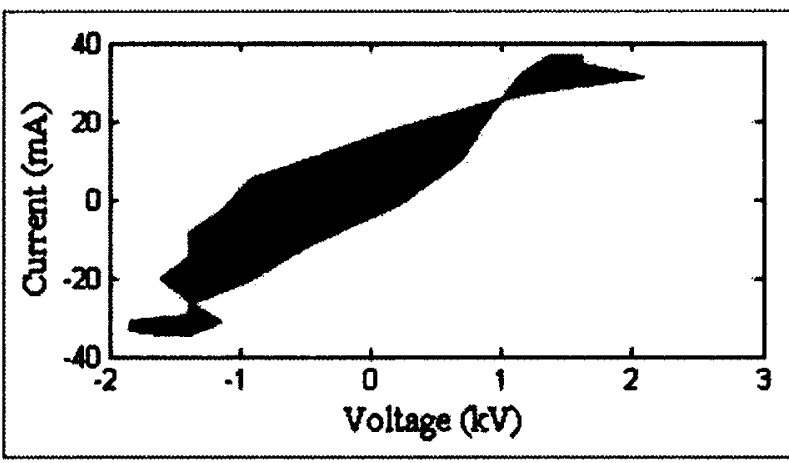

Figure 6.9: Sample voltage-current hysteresis of the first mode, at atmospheric pressure
A sample voltage-current hysteresis curve of the first discharge mode is shown in Figure 6.9. It should be noted that although the 'noise' near peak voltages was not accounted for, it does not make a significant contribution to 
the discharge power. The derivative of the discharge current with voltage indicates the plasma conductance. According to Ohm's Law,

$$
d I=\Gamma(t) d V
$$

where $\Gamma(t)$ is the time-dependent conductance.

For the hysteresis curve in Figure 6.9, $d I / d V$ rates indicate that plasma conductance is mostly positive. This characterizes the Ohmic (resistive) nature of the first mode of discharge, where most of the discharge energy is absorbed by the gas. For this sample, the $d I / d V$ rates are approximately 50 and $-30 \mathrm{~mA} / \mathrm{kV}$ during the polarity transition.

Figure 6.10 shows the average discharge power of 50 plasma support pulses for all 8 ignition events, for a higher pressure range. The average discharge power is approximately $1470 \mathrm{~W}$, two orders of magnitude higher than the power setting. The elevated air pressure is the only factor contributing to this sudden rise in the power discharged. It should be noted that due to significant voltage-current phase shift, most of the discharge power is reflected in the circuit.

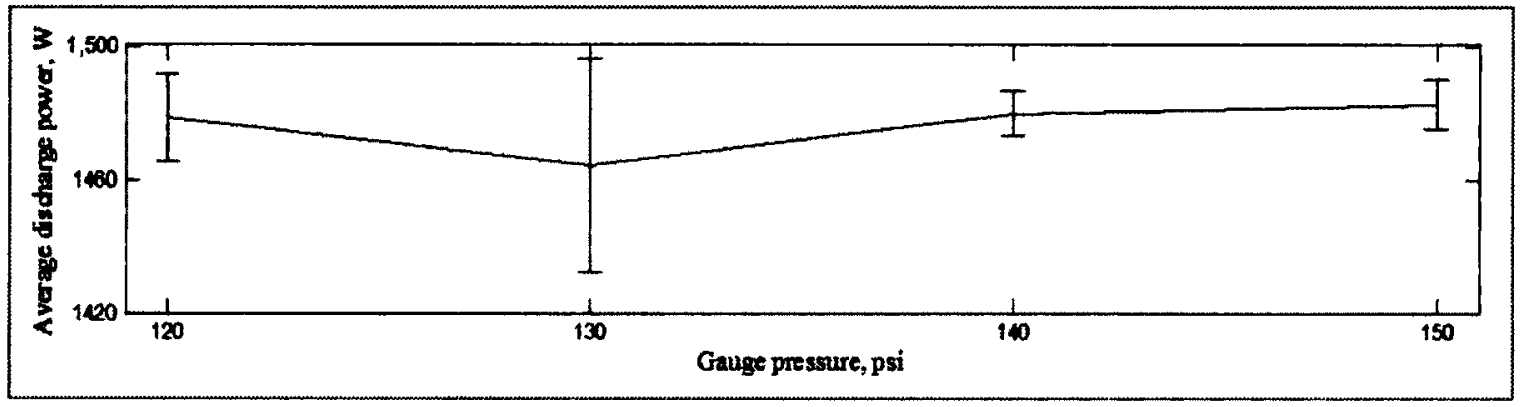

Figure 6.10: Average discharge power in the second discharge mode for the pressures tested Error bars shows 1 standard deviation for the 8 ignition events 
A sample voltage-current hysteresis curve of the second discharge mode is shown in

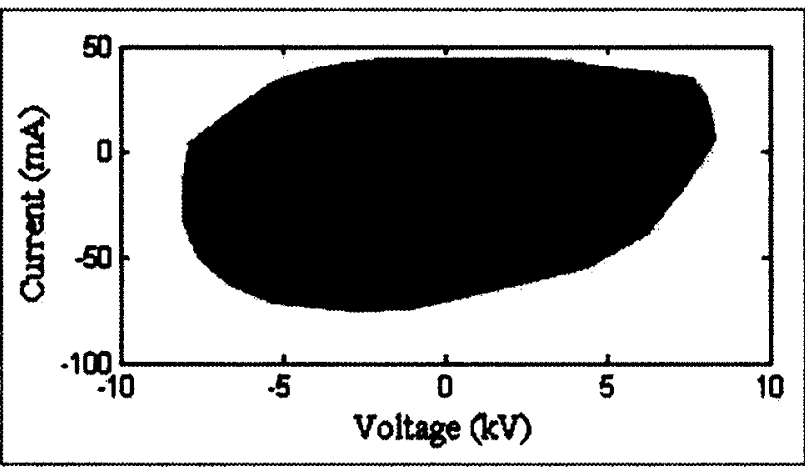

Figure 6.11: Sample voltage-current hysteresis of the second mode, at 120 psi gauge pressure
Figure 6.11. There is no oscillatory behavior observed in this mode. The enhanced area is attributed to the large capacitive impedance. For the hysteresis curve in Figure $6.11, d I / d V$ rates indicate that the overall plasma conductance is lower in this mode. This characterizes the capacitive nature of this mode of discharge, where most of the discharge energy is reflected back to the source. For this sample, the $d I / d V$ rates are only significant near the peak voltage amplitudes, and are approximately 50 and $-80 \mathrm{~mA} / \mathrm{kV}$.

At 110 psi gauge air pressure, both modes of discharge coexist. This is illustrated in Figure 6.12, on a logarithmic scale of the average discharge power for each of the 8 ignition events. The voltage-current waveforms are similar to the modes shown in Figure 6.4 and Figure 6.5. Note that ignition number 1, 3 and 4 represent the second discharge mode, whereas the remaining ignitions represent the first discharge mode.

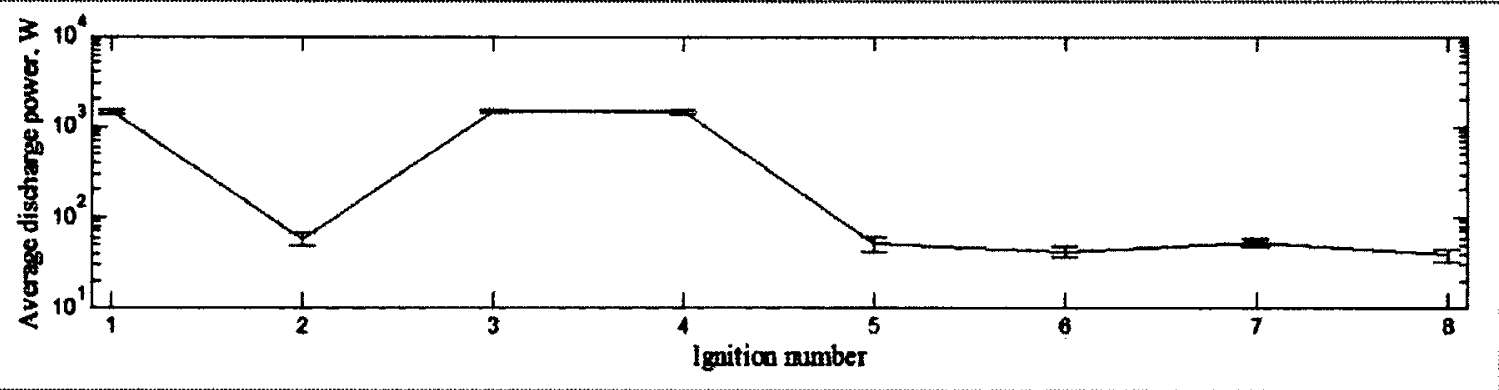

Figure 6.12: Average discharge power at 110 psi gauge pressure, illustrating both modes of discharge Error bars show 1 standard deviation for the 8 ignition events 


\subsubsection{Power factor of the plasma support phase}

All previously computed discharge powers are apparent powers (the sum of the resistive and reactive powers). The capacitive and inductive impedances of the plasma bulk and sheaths limit the resistive power that is absorbed by the gas medium.

The average real power of the 8 SPDI ignitions for atmospheric to 150 psi gauge pressure is plotted in Figure 6.13. The average real power is determined by the formula

$$
P_{\text {avg }}=1 / T \int V(t) I(t) d t
$$

where $T$ is the pulse period ( $28 \mu$ s for the SPDI pulse). The discharge mode transition at 110 psi gauge air pressure is evident. Otherwise, discharge power is nearly uniform in the two modes.

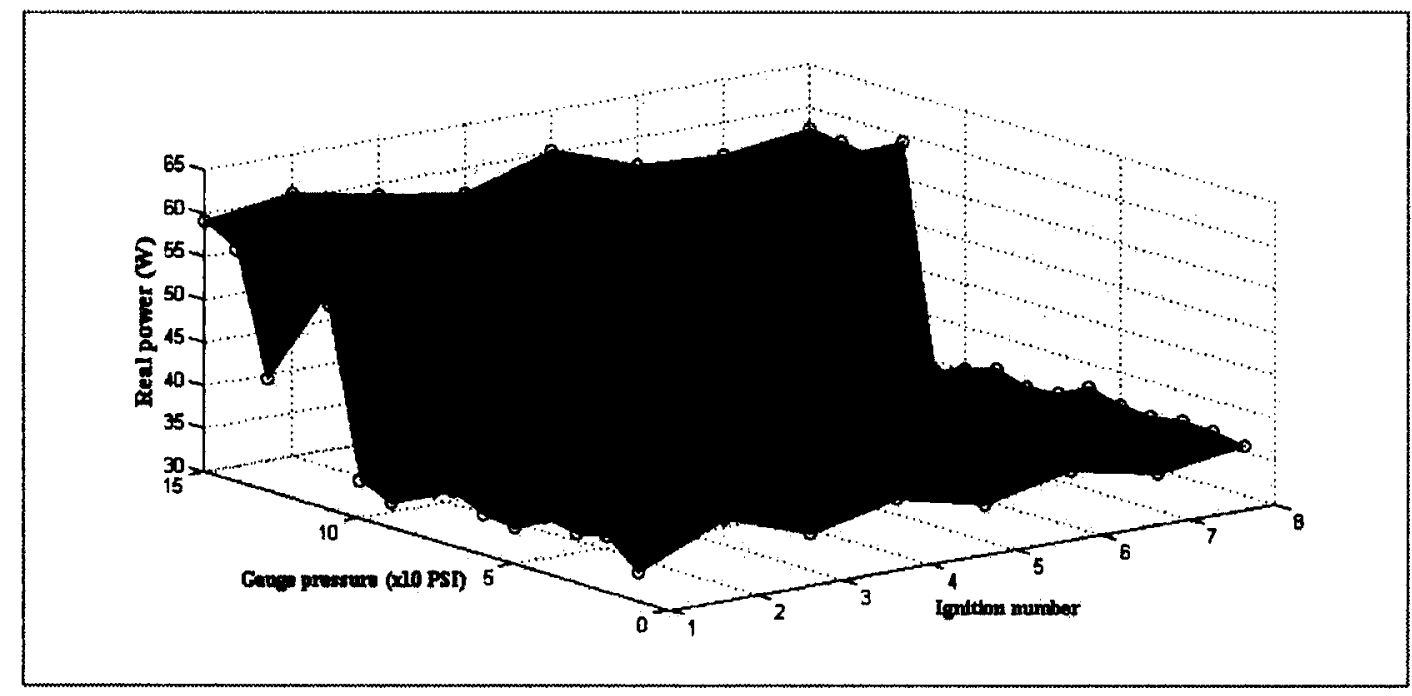

Figure 6.13: Average real power of the plasma support phase of SPDI for the pressures tested

The average power factors of the $8 \mathrm{SPDI}$ ignitions are plotted in Figure 6.14. Most of the discharge power $(\sim 70 \%)$ is absorbed by the gas in the first mode; this is a glow discharge 
as will be shown subsequently. However, in the second mode (postulated as streamers),

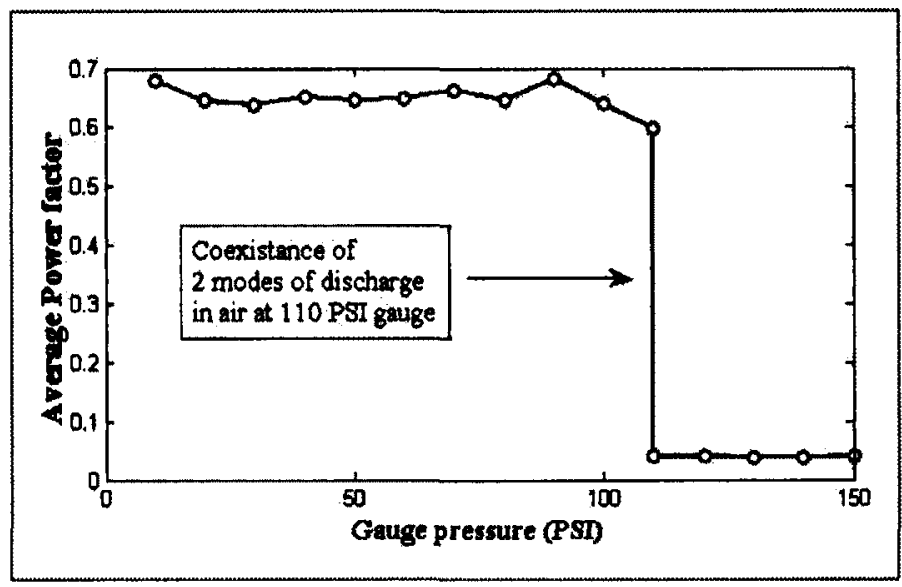

Figure 6.14: Average power factors of SPDI for the pressures tested most of the discharge power $(\sim$ $95 \%)$ is reflected; this is due to the sheath disappearance as will be shown subsequently. The coexistence of the two modes at 110 psi gauge air pressure indicates that collision frequency plays an important role in the overall

discharge impedance. Recall that for non-thermal plasmas, the electron-gas collision frequency is most relevant.

\subsection{SPDI Comparison to Experiments in the Literature}

The plasma support phase of SPDI in atmospheric air is an example of an atmospheric pressure AC glow discharge (aka. an atmospheric pressure plasma jet, APPJ). Most APPJs are tested at a frequency of $13.56 \mathrm{MHz}$, which is the most common frequency for plasma processes. This frequency is much higher than the ion frequency (Fridman, 2008), and in consequence, ions are trapped and positive sheaths forms near each electrode; therefore conduction in the positive column is strictly due to electrons. However, this is not the case for SPDI, since its much lower frequency of $35.7 \mathrm{kHz}$ cannot trap the ions. 


\subsubsection{The atmospheric pressure plasma jet (APPJ)}

Figure 6.15 illustrates 2 modes of the RF APPJ developed by Yang et al., (2005). In the

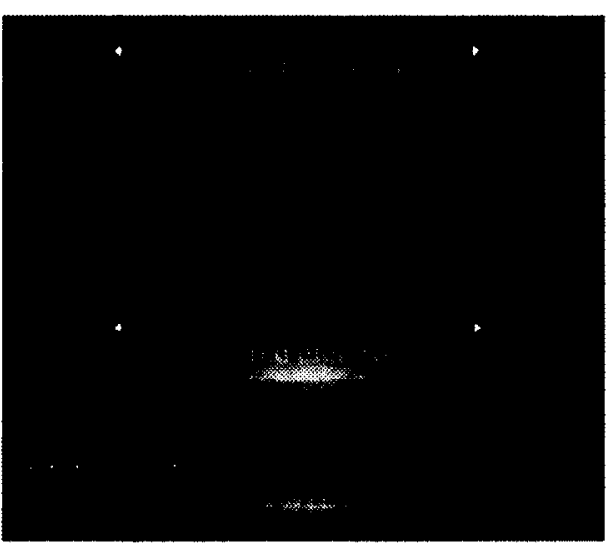

Figure 6.15: The 2 modes of an RF APPJ discharge $\alpha$-mode, a uniform glow is observed throughout the gap due to bulk ionization. In the $\gamma$-mode, glow intensity is enhanced near the electrodes due to secondary electron emission. The authors observe that the $\alpha-\gamma$ transition occurs for a threshold discharge current density that characterizes the sheath breakdown.

The voltage-current hysteresis curve of the $\alpha$-mode glow discharge, as shown by Farouk (2009), resembles the second mode of SPDI due to its large capacitive impedance; however, it is very important to note that SPDI at high pressure cannot sustain the glow discharge, and its capacitive impedance is due to a thermal instability due to the rising pressure.

Figure 6.7 and the second image in Figure 6.15 (Yang et. al, 2005) show much resemblance, since intensive collisional de-excitation is observed near the electrodes and low uniform glow is observed throughout the rest of the gap. This indicates that plasma created by SPDI is a $\gamma$-mode AC APPJ.

The APPJ device designed by Kim et al. (2009) operates at $50 \mathrm{kHz}$ or $13.56 \mathrm{MHz}$ frequency. Their oscilloscope readings indicate that the $\mathrm{kHz}$ frequency plasma can dissipate $50 \%$ more power, but requires significantly higher voltages (order of $10^{3} \mathrm{~V}$ 
instead of $10^{2} \mathrm{~V}$ for RF). This is consistent with the oscilloscope readings presented in this chapter. Furthermore, the authors determine that the addition of a ground copper electrode acts as a dielectric barrier, and allows stable discharges at higher currents that ultimately intensify the production of $\mathrm{O}, \mathrm{OH}$ and $\mathrm{N}_{2}{ }^{+}$radicals. On a similar basis, the ground electrode in SPDI allows to create stable plasma discharges at various pressures.

The thermal stability of the APPJ is maintained by the sheath. However, as illustrated in (Fridman, 2008) the critical power threshold is an exponential function of $\mathrm{R}$, where $\mathrm{R}$ is the square of the ratio of the sheath voltage to the plasma voltage. Hence higher power APPJ can be stabilized if sheath voltage increases. SPDI's transition at high pressure is a result of sheath disappearance caused by the thermal instability. This topic is analyzed in Chapter 8 via an ordinary differential equation model of SPDI.

\subsection{Remarks on experimental testing}

Oscilloscope data of SPDI discharges in air display 2 different modes of discharge, postulated as glow and streamer discharges. The first mode of discharge transmits most of its power into the gas. The second mode of discharge is characterized by significant DC current bias and mostly reactive power in the circuit. For this reason, the second mode of discharge has very low luminosity that cannot be seen with the naked eye. Pressure is a principal factor of the mode transition, and co-existence of the discharge modes is possible at a threshold pressure. 


\subsection{Fundamental Assumptions relevant to Modeling SPDI}

Fundamental assumptions of the SPDI discharge in air are presented and discussed in the following subsections. The 1D plasma discharge model presented in Chapter 7 is built upon the validity of these assumptions.

\subsubsection{High air pressure}

The high pressure nature of SPDI implies that plasma is highly collisional. First order estimate of the similarity parameter $\frac{v_{e n}}{P}$ in air is $4 \cdot 10^{9} \mathrm{~s}^{-1} \cdot \operatorname{torr}^{-1}$ (Fridman, 2008), while the plasma frequency is in the $\mathrm{GHz}$ range for atmospheric pressure glow discharges (see Chapter 2). The driving frequency of SPDI (converted to radians) is

$$
\omega_{S P D I}=2.244 \cdot 10^{5} \frac{\mathrm{rad}}{\mathrm{s}} .
$$

Since the electron-gas collision frequency is much higher than the plasma frequency or the driven frequency, the real relative permittivity of SPDI plasma, as given by Equation 3.17 and using Equation 3.14 , is $\varepsilon_{r e l} \approx 1$. This means that this type of plasma has significant dielectric loss (large imaginary component in Equation 3.17), and most conductivity is due to the charged particles. Therefore we will make the following assumptions in our models, consistent with a SPDI discharge in high pressure air.

\subsubsection{The electrostatic approximation in SPDI plasma}

For the glow discharge, the estimated electron number density is on the order of $10^{15}$ to $10^{21} \mathrm{~m}^{-3}$ (see Chapter 2). Plasma conductivity can be found using Equation 3.16, using the electron-neutral collision frequency in air at atmospheric pressure given by Fridman (2008). This conductivity is in the rage $10^{-7}$ to $10^{-1} \mathrm{Ohm}^{-1} \mathrm{~cm}^{-1}$. Using Equation 3.20 , the skin depth for the postulated glow mode of the plasma support phase of SPDI is on the 
order of $10^{5}$ to $10^{2} \mathrm{~cm}$; this is much larger than the discharge gap size of $0.1 \mathrm{~cm}$. Hence the electrostatic approximation applies for the plasma produced by SPDI and simulation complexity is reduced significantly.

\subsubsection{Polarization field in SPDI plasma}

It was previously shown that the plasma produced by SPDI has a real permittivity constant of unity. Hence the polarization field due to space charge is given by the Poisson equation, a simplified form of Equation 3.23; if assuming single charge ions, then

$$
\nabla^{2} \mathrm{~V}=-\mathrm{e} / \varepsilon_{o} \cdot\left(n_{p}-n_{n}-n_{e}\right)
$$

This equation determines the electrostatic field force and will be used in Chapter 7 .

\subsection{Hypothesis of SPDI}

Recall that the plasma support phase is the last stage of SPDI. Plasma created during this period is when the flame radius, $R_{\text {Flame, }}$ is larger than the critical ignition radius, $R_{C}$,

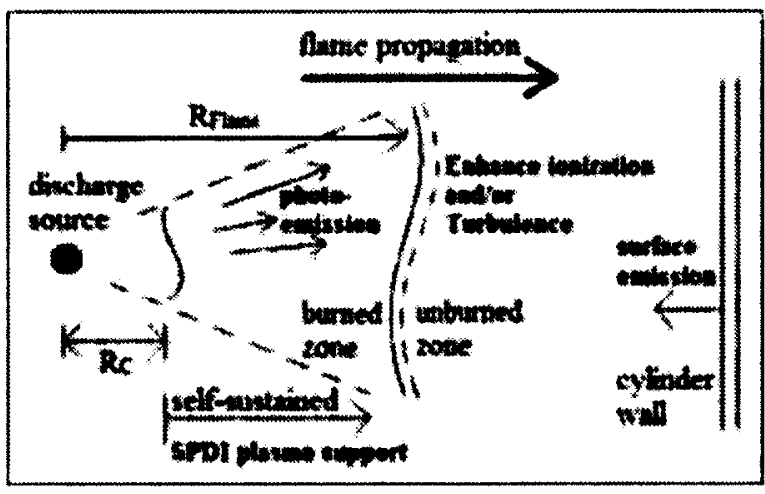

Figure 6.16: Hypothesis for excitation of the flame front using SPDI required to self-sustain combustion. This is depicted in Figure 6.16, as the burned (post-flame) zone, which is mainly composed of $\mathrm{CO}_{2}$ gas and $\mathrm{H}_{2} \mathrm{O}$ vapor.

Assuming SPDI produces plasma discharges such that molecules are excited ahead of the flame front (via photo-

excitation, or energy exchange channels such as vibrational exchange), then production of atomic and radical species can be enhanced due to lower energy required for their dissociation. Recall that discharges such as streamers require photo-ionization to be 
sustained, and experiments in the literature indicate flame acceleration and highly turbulent flame fronts for long duration corona discharges.

\subsubsection{Photo-excitation in $\mathrm{CO}_{2}$ and $\mathrm{H}_{2} \mathrm{O}$}

Using the photo-absorption cross sections for $\mathrm{CO}_{2}$ and $\mathrm{H}_{2} \mathrm{O}$ in (Gallagher et al., 1988), and assuming the following,

- Gas temperature corresponding to the auto-ignition of gasoline given in (Elert, 2012), at pressure corresponding to a typical ignition timing of $20^{\circ} \mathrm{BTDC}$ given in (Giordano, 2012), and

- The Ideal Gas Law, the mean free path of molecular excitation by high UV photons is less than $0.02 \mathrm{~cm}$. Hence photo-excitation ahead of the flame front is unlikely. It should be noted that mean free paths for higher energy photons (i.e. $x$-rays) are larger; however, emission spectra experiments of SPDI is required to confirm the possibilities of photo-excitation.

\subsubsection{Energy exchange channels in $\mathrm{CO}_{2}$ and $\mathrm{H}_{2} \mathrm{O}$}

Recall that in non-thermal plasmas, the EEDF is responsible for distributing the energy among different energy channels within the gas molecules. As shown in Figure 6.17 (Fridman \& Kennedy, 2004), vibrational excitation and dissociative attachment dominate in $\mathrm{H}_{2} \mathrm{O}$ vapor for electron temperatures of 1 to $3 \mathrm{eV}$, whereas electronic excitation is only significant for electron temperature above $3 \mathrm{eV}$. In $\mathrm{CO}_{2}$, the threshold between vibrational and electronic excitation is around a reduced electric field of $100 \mathrm{Td}$. It should be noted that since vibrational excitation occurs over a wide range of electron temperatures, the fraction of high energy electrons required for electronic excitation is 


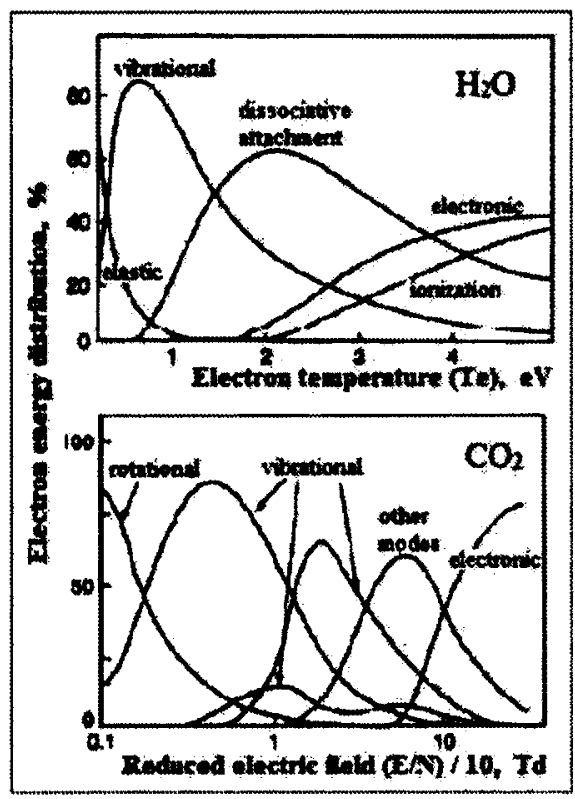

Figure 6.17: Electron energy distribution in $\mathrm{H} 2 \mathrm{O}$ and $\mathrm{CO} 2$ reduced. Consequently, this promotes additional

vibrational excitation at lower electron temperatures.

Figure 6.17 indicates that for plasma discharges in $\mathrm{H}_{2} \mathrm{O}$ and $\mathrm{CO}_{2}$, most of the energy exchange is due to vibrational, V-V transfer; this is a resonant process where cross sections can be higher than the elastic cross sections (Fridman \& Kennedy, 2004). The vibrational exchange of energy is important in molecular dissociation, since collisional quenching of

a vibrational state and a low temperature electron may have enough energy to dissociate neighbor molecules. An example is the quenching of $\mathrm{N}_{2}$ vibrational states, which are believed to be the major source of $\mathrm{OH}$ production (Van Veldhuizen $\&$ Rutgers, 2001). Here, we postulate that SPDI plasma causes more ionization at the flame front through vibrational energy exchange channels in the post-flame zone. However, such a hypothesis is very difficult to validate. 


\section{Chapter: Plasma Model Formulation}

This chapter presents a numerical model of 1-dimensional plasma simulations for nonthermal discharges such as SPDI. Here, we assume that plasma is highly non-thermal so gas heating is ignored. Since no kinetic energy is gained via elastic gas scattering, most of discharge energy must transfer to the electron temperature and the internally excited states of the gas.

\subsection{Purpose of the Model and the Modeling Approach}

The purpose of the plasma model is to determine the spatial and temporal distributions of electric field, electron/ion density, and electron mean energy. The governing equations presented in section 7.4 are obtained from the general Boltzmann equation presented in section 4.5 using non-equilibrium electron distribution functions, and electron transport/rate coefficients calculated by Bolsig+.

\subsection{The 1D Approximation and Importance of 3D effects}

For simplicity, only variations in one-dimension, the axial direction of discharge, are considered. This imposes a serious limitation since the discharge gap should be infinitesimal compared to the size of the electrodes. However, the model can still be used as a first step to understanding plasma behavior qualitatively.

It should be noted that in order to model SPDI accurately, 3D spatial variations cannot be ignored. Breakdown experiments in air by Mardiguian (2009) show that when the discharge gap approaches the electrode size, breakdown voltages are almost an order of magnitude higher. This is consistent with breakdown voltages in SPDI. Experimental 
data indicate breakdown voltages that are in the $10 \mathrm{kV}$ range, whereas breakdown voltage between parallel plates in atmospheric air is on the order of $1 \mathrm{kV}$ (see Figure 4.1). A 1D model ignores radial diffusion, therefore over-estimate the overall space charge field. Furthermore, the 3D geometry of spark spark-plugs enhances the electric field around the powered electrode, which favors the sparking condition rather than Townsend breakdown (see section 4.2.1).

\subsection{Model Assumptions}

A complete model of plasma kinetics is very complex and approximations must be made to reduce problem complexity, simplify the governing equations, and the computational time. The assumptions outlined below are specific to modeling SPDI plasma in air in one dimension.

1) Momentum and energy of air molecules and excited species is ignored. Also, it is also assumed that gas density is constant. To account for the gas fluid dynamics, a Navier-Stokes equations module is required and is not considered here for simplicity. 2) Air is dry and composed of $79 \% \mathrm{~N}_{2}$ and $21 \% \mathrm{O}_{2}$. The Van der Waals equation presented in (Zevenhoven, 2001) is used to estimate air density over the pressure range in Chapter 6.

3) Only single-charge ions are considered; it is assumed that positive ions have the averaged mass of $\mathrm{O}_{2}{ }^{+}$and $\mathrm{N}_{2}{ }^{+}$, and negative ions have the mass of $\mathrm{O}_{2}{ }_{2}^{-}$. These ions are common in air discharges.

4) Ions respond to the actual electric field fluctuations in the discharge gap. This is assumed valid for SPDI due to its $\mathrm{kHz}$ frequency. Effective electric field must be derived if the driven frequency is higher than the ion-neutral collision frequency, which is the case for discharges at $\mathrm{MHz}$ frequency.

5) The electron and ion momentum is simplified to the drift-diffusion approximation, which is very common in fluid simulations of plasmas. 
6) Momentum transfer is due to direct electron impact ionization, uniform photoionization, electron attachment and detachment, electron-ion and ion-ion recombination. Ionization and attachment rates are functions of the mean electron energy, whereas recombination and detachment rates are constant.

7) The total electron energy is equal to its thermal energy; thus the energy drift is ignored. This assumption is frequently used in plasma simulations.

8) Energy transfer is due to elastic scattering of electrons-air molecules and inelastic collisions of electrons with air molecules. Elastic and inelastic collision rates are functions of the mean electron energy.

9) Ion diffusivity is computed from ion mobility, using Einstein's relation, and electron diffusivity is computed from its mean energy. This is a common approach.

10) Thermal conductivity of electrons in air is a correction to the thermal conductivity of the free electron model given by the Drude model explained in (Tritt, 2005). This correction is based on the paper by Balcon (2007).

11) Ion temperature and transport coefficients are obtained from the local field approximation.

12) Electron temperature and transport coefficients are computed via $L X \mathrm{Cat}^{5}$ for nonequilibrium distributions. Recall that this is the typical approach to include non-local effects based on the known results of the local field.

\subsection{Governing Equations}

Following the assumptions above, the model reduces to the solution of 4 conservation equations: an equation for the transport of each type of particle (electrons, positive and negative ions) and one equation for the conservation of mean electron energy.

Conservation of electron density is given by

\footnotetext{
${ }^{5}$ Recall that LXcat is an open-access website that uses the Bolsig+ solver
} 


$$
\begin{aligned}
\frac{\partial n_{e}}{\partial t}+\frac{\partial}{\partial x}\left(n_{e} v_{e}\right) & -\frac{\partial}{\partial x}\left(D_{e} \frac{\partial n_{e}}{\partial x}\right) \\
& =(\alpha-\beta)\left|n_{e} v_{e}\right|-\eta_{e} n_{e} n_{p}+\delta n_{n} N+S_{p h}
\end{aligned}
$$

where $v_{e}$ is the electron drift velocity, $D_{e}$ is the electron diffusion coefficient, $\alpha, \beta, \eta_{e}$, and $\delta$ are the electron impact ionization, attachment, electron-ion recombination, and detachment coefficients respectively, and $\mathrm{S}_{\mathrm{ph}}$ is the photo-ionization source; $n_{p}, n_{n}$ and $N$ are the positive, negative ion, and air density respectively.

Conservation of positive ion density is given by

$$
\begin{aligned}
\frac{\partial n_{p}}{\partial t}+\frac{\partial}{\partial x}\left(n_{p} v_{p}\right) & -\frac{\partial}{\partial x}\left(D_{i} \frac{\partial n_{p}}{\partial x}\right) \\
& =\alpha\left|n_{e} v_{e}\right|-\eta_{e}\left(n_{e} n_{p}\right)-\eta_{i}\left(n_{n} n_{p}\right)+S_{p h}
\end{aligned}
$$

where $v_{p}$ is the positive ion drift velocity, $D_{i}$ is the ion diffusion coefficient, and $\eta_{i}$ is the ion-ion recombination coefficient.

Conservation of negative ion density is given by

$$
\begin{aligned}
\frac{\partial n_{n}}{\partial t}+\frac{\partial}{\partial x}\left(n_{n} v_{n}\right) & -\frac{\partial}{\partial x}\left(D_{i} \frac{\partial n_{n}}{\partial x}\right) \\
& =\beta\left|n_{e} v_{e}\right|-\eta_{i}\left(n_{n} n_{p}\right)-\delta\left(n_{n} N\right)
\end{aligned}
$$

where $v_{n}$ is the negative ion drift velocity and $D_{i}$ is the ion diffusion coefficient.

Equations 7.1 through 7.3 describe the general form of unsteady convection-diffusion. The first term of LHS is the transient term, the second term is convection, and the third term is diffusion. The sum of the RHS terms is the net source. The convection-diffusion flux, $F$, is the sum of the second and third term on the LHS of Equations 7.1 through 7.3; this represents the transport of momentum 


$$
F= \pm \mu E n-D \nabla n
$$

where $\mu$ is the particle mobility.

The electron energy conservation equation is given by

$$
\frac{\partial\left(n_{e} \bar{\varepsilon}\right)}{\partial t}+\nabla \cdot\left(\left(\bar{\varepsilon}+k T_{e}\right) F_{e}-K_{e} \nabla T_{e}\right)=-e \boldsymbol{F}_{e} \cdot \boldsymbol{E}-\sum R U
$$

where $\bar{\varepsilon}$ is the average thermal energy, $k$ is Boltzmann constant, $T_{e}$ is the electron temperature, $F_{e}$ is the electron flux, $K_{e}$ is the electron coefficient of thermal conductivity, $e$ is the elementary charge, and $\mathbf{E}$ is the electric field. The last 2 terms on the LHS in Equation 7.5 represent Joule heating, which is the electron acceleration in the electrostatic field; and the total rate of energy loss due to elastic and inelastic collisions, where $R$ is the reaction rate constant, and $U$ is the energy exchange.

The total rate of collisional energy exchange can be decomposed into 2 terms: the elastic energy transfer due to electron-gas molecule scattering, and inelastic energy transfer due to electron-neutral collisions that results in internally excited states. Thus, in Equation 7.5 we can use

$$
\sum R U=3 v_{e n} \frac{m_{e}}{M} n_{e} k\left(T_{e}-T_{g a s}\right)+\sum_{\text {inel }} N n_{e} k_{i}(\bar{\varepsilon}) U_{i}
$$

where $v_{e n}$ is the electron-neutral collision frequency, $\frac{m_{e}}{M}$ is the mass ratio of the electron and air molecule, $T_{\text {gas }}$ is the constant air temperature, and $k_{i}(\bar{\varepsilon})$ and $U_{i}$ are the reaction rate and energy exchange due to specified inelastic collisions. 
The energy equation can be converted in a form similar to the equation for electron density conservation; Balcon (2007) derives this expression. If energy density of electrons is given by

$$
n_{\varepsilon}=n_{e} \bar{\varepsilon}
$$

where the mean electron energy of the EEDF is given by

$$
\bar{\varepsilon}=\frac{3}{2} k T_{e}
$$

then the energy equation reduces to

$$
\frac{\partial n_{\varepsilon}}{\partial t}+\nabla F_{\varepsilon}=-e F_{e} \cdot E-\sum R U
$$

where $F_{\varepsilon}$ is the flux of energy density

$$
\boldsymbol{F}_{\varepsilon}=-n_{\varepsilon} \mu_{\varepsilon} \boldsymbol{E}-D_{\varepsilon} \nabla n_{\varepsilon}
$$

Following the derivation by Balcon (2007), mobility and diffusivity of the electron energy become $5 / 3$ times the electron mobility and diffusivity in physical space.

\subsection{Transport and Rate Coefficients}

Recall that the electron energy is solved under the assumption of a non-equilibrium distribution in order to include non-local effects. In this model, electron mobility, diffusion coefficients, temperature, and ionization and attachment rates are obtained from Morgan's database in LXcat (MORGAN database, 2012). This database includes elastic scattering of the electron with $\mathrm{N}_{2}$ and $\mathrm{O}_{2}$ molecules, and 40 inelastic collisions of vibrational and electronic excited states of $\mathrm{N}_{2}$ and $\mathrm{O}_{2}$.

Figure 7.1 and 7.2 are plots of the electron mobility and inelastic energy loss data in the MORGAN database. Figure 7.1 below shows the electron mobility as function of electron mean energy. Figure 7.2 illustrates the overall rate of energy loss due to inelastic 
collisions, as a function of mean electron energy. Bolsig + first computes the EEDF in order to determine the reaction rate (the cross sections of various reactions are built-in).

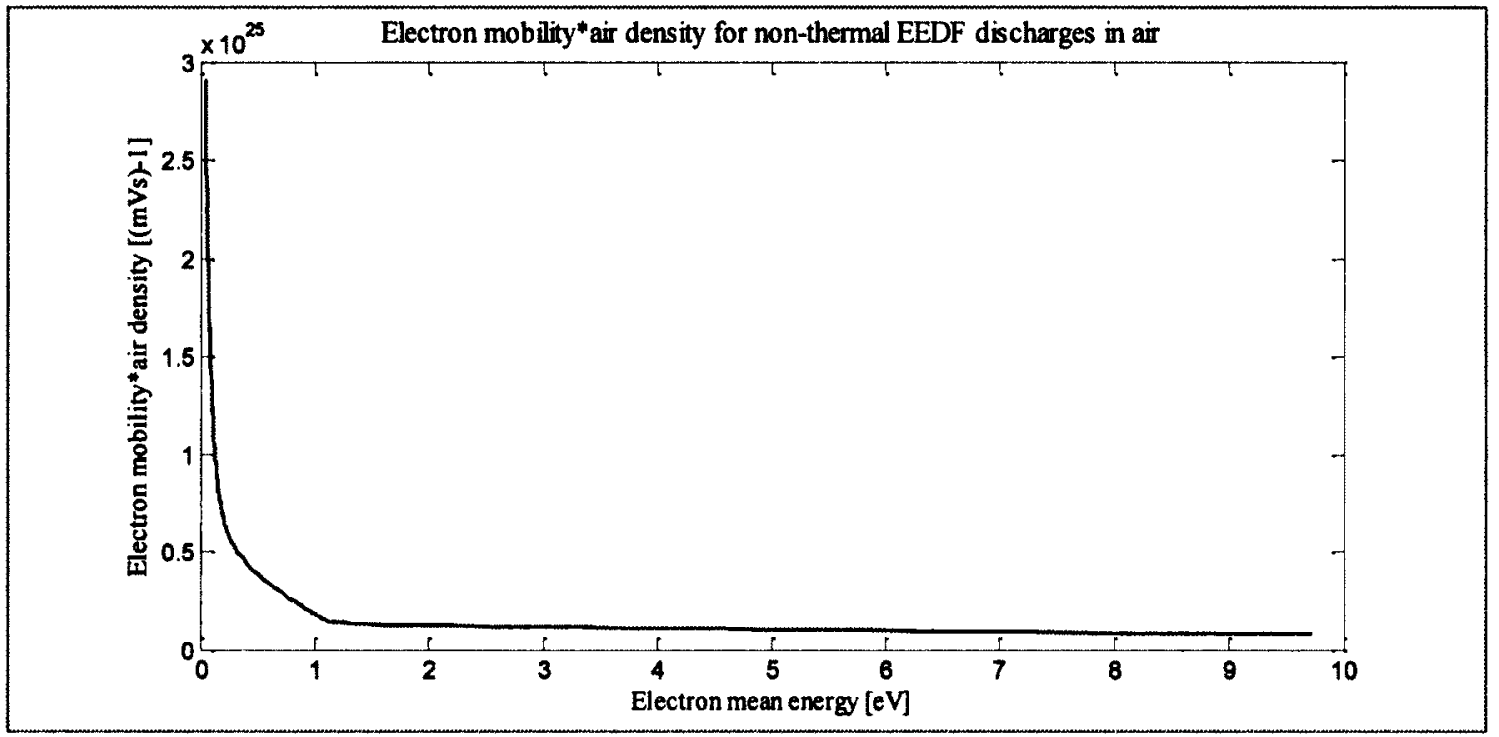

Figure 7.1: Sample plot of the reduced electron mobility

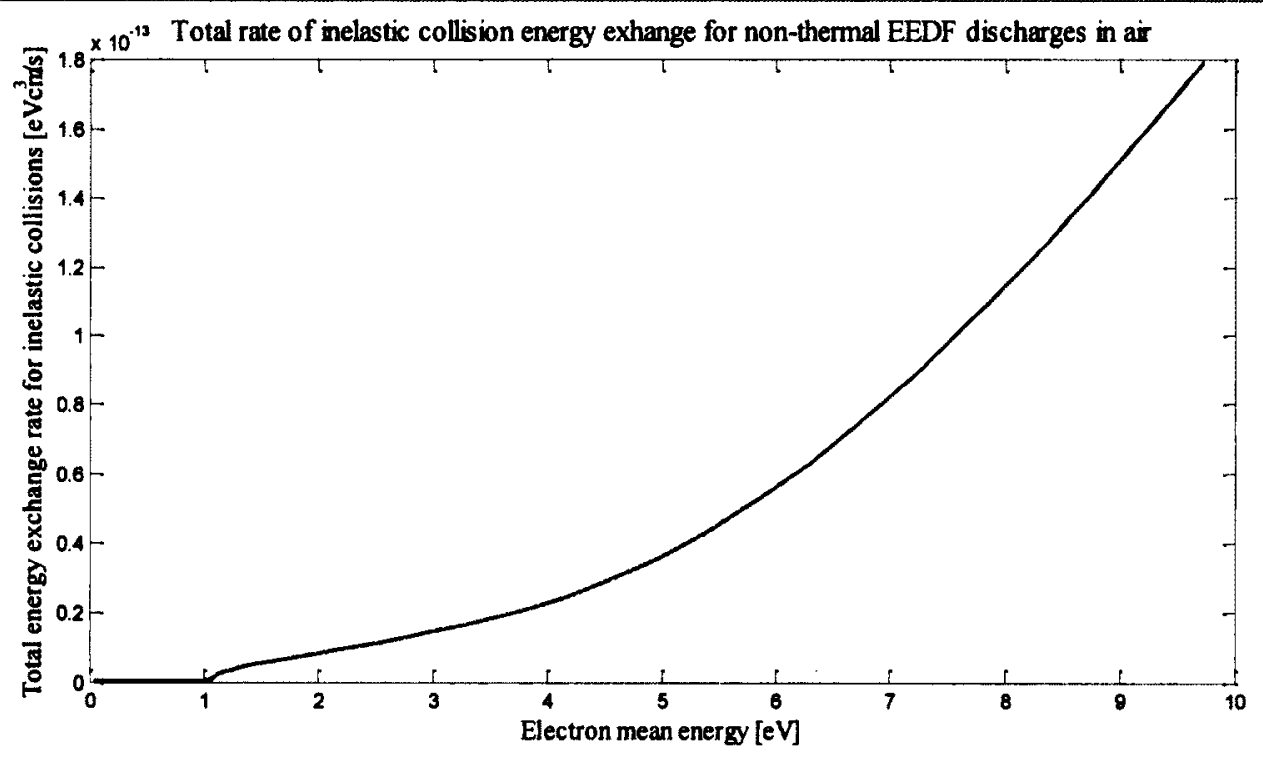

Figure 7.2: Sample plot of the total rate of electron energy loss due to inelastic collisions in air 
Constant reaction rates are assumed for electron detachment, electron-ion and ion-ion recombination folowing (Capitelli et al., 2000), and background photo-ionization

- Electron detachment rate, $\delta=1 / 2\left[2.8 \cdot 10^{-10}+2 \cdot 10^{-9}\right] \mathrm{cm}^{3} \mathrm{~s}^{-1}$

- Ion - ion recombination, $\eta_{i}=2 \cdot 10^{-7} \mathrm{~cm}^{3} \mathrm{~s}^{-1}$

- Electron - ion recombination,

$$
\eta_{e}=1 / 2\left[1.8 \cdot 10^{-7}\left(T_{g a s} / T_{e}\right)^{0.39}+1.6 \cdot 10^{-7}\left(T_{g a s} / T_{e}\right)^{0.55}\right] \mathrm{cm}^{3} \mathrm{~s}^{-1}
$$

- Photo-ionization:

$$
S_{p h}=10^{7 P} / P_{o} \mathrm{~cm}^{-3} \mathrm{~s}^{-1} \text { if } 1 / d \int_{0}^{d} E_{\text {total }} d x>2 \bar{E}
$$

$$
10^{6} \mathrm{P} / \mathrm{P}_{o} \mathrm{~cm}^{-3} \mathrm{~s}^{-1} \text { if } 1 / d \int_{0}^{d} E_{\text {total }} d x<2 \bar{E}
$$

Estimation of the photo-ionization source term at $P_{0}$ (atmospheric pressure) is $10^{6} \mathrm{~cm}^{-3} \mathrm{~s}^{-1}$ (Smith et al., 2011). For the model described here, it is assumed that this source term scales proportionally with pressure, and increases by an order of magnitude if the space averaged electric field is higher than twice the average electric field (meaning that electric fields are highly distorted due to local space charges). This assumption is used to account for faster breakdown that is caused at elevated pressures (see Chapter 4). It should be noted that the uniform photo-ionization source is a poor assumption. For instance, streamer discharges must incorporate a more computationally demanding expression for the photo-ionization source as presented by Quast and Lalic (2009), and described in APPENDIX A.

The electron-neutral collision frequency can be obtained from the electron mobility,

$$
v_{e n}=\frac{e}{\mu_{e} m_{e}} .
$$


The ion mobility and temperature in air discharges are approximated as functions of the local reduced electric field (Koh \& Park, 2003) and (Capitelli et al., 2000)

$$
\begin{gathered}
\mu_{i}=\frac{1}{N}\left(8.65+0.015\left(\frac{E}{N}\right) 10^{17}\right) \cdot 10^{19} \\
T_{i}=T_{\text {gas }}+\frac{2 m_{i}}{3 k}\left(\mu_{i} E\right)^{2}
\end{gathered}
$$

In Equations 7.12 and 7.13, the reduced electric field $E / N$ must be in $V / \mathrm{cm}$, the ion mass $m_{i}$ in $\mathrm{kg}$, and gas temperature $T_{\text {gas }}$ in $\mathrm{K}$; then the resulting ion mobility is in $\mathrm{cm}^{2} / \mathrm{sV}$ and ion temperature is in $\mathrm{K}$.

The ion diffusion coefficient is obtained from the Einstein relation (Lymberopoulos \& Economou, 1996), where the ion has elementary charge

$$
D_{i}=\frac{k \mu_{i} T_{i}}{e} .
$$

Following the Drude model of the free electron by Tritt (2005), the thermal conductivity coefficient of electron is related to its diffusivity. Similar expression can be used for the thermal conductivity of electrons in air as shown by Balcon (2007),

$$
K_{e}=\frac{5}{2} k n_{e} D_{e}
$$

\subsection{The Numerical Code}

The model is implemented in Matlab via the finite volume method (FVM), which is explained by Versteeg and Malalasekera (2007). This is the preferred numerical method for fluid conservation equations. The 1D discretization is shown in Figure 7.3. Scalar quantities (particle density, potential, electron mean energy, mobility, diffusivity) are defined at the nodes, and vector quantities (electric field, drift velocity, flux) are defined at the faces. The physical boundaries portray the potential difference, where the grounded 
electrode is $\mathrm{V}_{\mathrm{G}}=0 \mathrm{~V}$. Electric fields at the boundaries, $\mathrm{E}_{\mathrm{BC}_{-} 1}$ and $\mathrm{E}_{\mathrm{BC} \_}$require special attention, which is discussed subsequently.

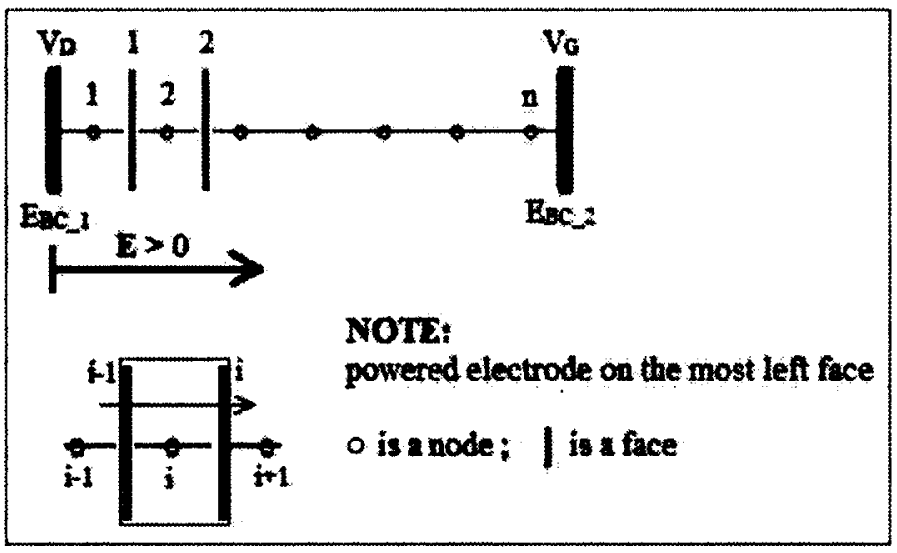

Figure 7.3: Spatial discretization via the staggered grid
The bottom of Figure 7.3 depicts

the flux through a control volume.

Flux corrected transport (FCT)

and exponential schemes (i.e.

Scharfetter-Gummel) are

commonly used to compute the

exponential scheme is used due to its simplicity; it has the form

$$
F_{i}=a_{i} n_{i}+b_{i} n_{i+1}
$$

where subscript $i$ represents the spatial discretized step, and coefficients $\mathbf{a}$ and $\mathbf{b}$ are functions of drift velocity and diffusivity that will be explained subsequently.

\subsubsection{The FVM approach}

In the finite volume method, the time-rate of change of mass (or number density for this plasma model) is equivalent to its net flux and source within the control volume. For the following discretized equations, $i$ subscripts represent spatial steps, and $k$ superscripts represent time steps.

Integrating the general unsteady convection-diffusion equation in one spatial dimension and time results in

$$
\int_{i}^{i+1} \int_{k}^{k+1} \frac{\partial n}{\partial t} d t d x+\int_{i}^{i+1} \int_{k}^{k+1} \frac{\partial}{\partial x} F(n, v) d t d x=\int_{i}^{i+1} \int_{k}^{k+1} S d t d x
$$


where $n$ is the particle density, $F(n, v)$ is the particle density flux, and $S$ is the net source due to collisions. Time integration of the particle density is computed via a relaxation parameter $f$ such that

$$
\int_{t}^{t+\Delta t} n_{i} d t=\left[f n_{i}^{k+1}+(1-f) n_{i}^{k}\right] \Delta t
$$

The relaxation parameter controls coupling between the implicit and explicit formulation. For $f=0$ the discretization scheme is explicit, and for $f=1$ the discretization scheme is fully-implicit. The discretization scheme and stability criteria that are used for this model are discussed subsequently.

\subsubsection{Solving the particle flux}

Plasma discharges have steep gradients of electric field and particle density. Low order schemes can converge near discontinuities but have large numerical error. On the other hand, high order schemes diverge near discontinuities, but provide less numerical error in smooth regions. The exponential scheme presented below can overcome these problems.

\subsubsection{Exponential scheme for drift-diffusion}

The exponential scheme obtains exact solutions for steady drift-diffusion equations. However, it can be implemented in unsteady simulations too (Versteeg \& Malalasekera, 2007). Flux, velocity and diffusivity are assumed to be constant through the control volume. This becomes the solution of a boundary-value problem, with the particle number density as the boundary conditions. A derivation of this scheme can be found in (Frensley, 2004).

The flux at face ' $i$ ', between nodes ' $i$ ' and ' $i+1$ ' of the staggered grid in Figure 7.3 is 


$$
\begin{gathered}
F_{i}=a_{i} n_{i}+b_{i} n_{i+1} \\
F_{i}=\left(\frac{-\overline{v_{d}} e^{P e}}{1-e^{P e}}\right)_{i} n_{i}+\left(\frac{\overline{v_{d}}}{1-e^{P e}}\right)_{i} n_{i+1}
\end{gathered}
$$

In Equation 7.20, Pe is the Peclet number, given by

$$
P e=\frac{ \pm\left|\overline{v_{d}}\right| \Delta x}{\bar{D}}
$$

where $\overline{v_{d}}$ and $\bar{D}$ are the average drift velocity and average diffusivity. The exponential scheme is equivalent to upwind convection at high Peclet number, where diffusivity is insignificant. However, the upwind drift-diffusion scheme always linearizes diffusivity, and thus it over-estimates diffusion for high Peclet numbers. This means that particles with high drift velocity such as electrons are not accurately modeled. For this model, the threshold at which diffusivity is neglected is at $|\mathrm{Pe}|=10$, which Versteeg \& Malalasekera (2007) describe as the Power Law limit.

Expansion of Equation 7.17, with flux given by Equation 7.19 and relaxation parameter given by Equation 7.18 gives

$$
\begin{aligned}
n_{i}^{k}\left[\frac{\Delta x}{\Delta t}+f a_{i+1}\right. & \left.-f b_{i}\right]+n_{i+1}^{k}\left[f b_{i+1}\right]+n_{i-1}^{k}\left[-f a_{i}\right] \\
& =n_{i}^{k-1}\left[\frac{\Delta x}{\Delta t}-(1-f) a_{i+1}+(1-f) b_{i}\right] \\
& +n_{i+1}^{k-1}\left[-(1-f) b_{i+1}\right]+n_{i-1}^{k-1}\left[(1-f) a_{i}\right] \\
& +\Delta x S_{i}^{k} .
\end{aligned}
$$

Equation 7.22 indicates that $n_{i}^{k}=f$ unction $\left(n_{i}^{k-1}, n_{i+1}^{k-1}, n_{i-1}^{k-1}, S_{i}^{k}, n_{i+1}^{k}, n_{i-1}^{k}\right)$ and can be computed via the Tridiagonal Matrix Algorithm (TDMA). 


$$
\begin{aligned}
& {\left[\begin{array}{cccccc}
A_{1}^{k} & A_{2}^{k} & 0 & 0 & & \\
A_{W}^{k} & A_{P}^{k} & A_{E}^{k} & 0 & \cdots & \\
0 & A_{W}^{k} & A_{P}^{k} & A_{E}^{k} & & \\
& \vdots & & \ddots & 0 \\
& 0 & & \cdots & A_{\max -1}^{k} & A_{\max }^{k}
\end{array}\right]\left[\begin{array}{c}
n_{1}^{k} \\
n_{2}^{k} \\
n_{3}^{k} \\
\vdots \\
n_{\max }^{k}
\end{array}\right]}
\end{aligned}
$$

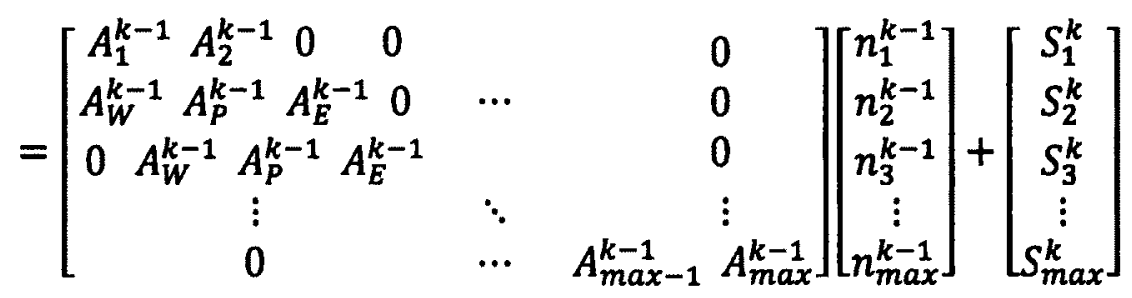

In the TDMA above, the square matrices represent the velocity field. These velocities are illustrated as the West Centre East coefficients, $A_{W} A_{P} A_{E}$ and $A_{W}^{k} A_{P}^{k} A_{E}^{k}$, which are calculated from Equation 7.20 based on the staggered grid shown in Figure 7.3. At the boundary nodes, $i=1$ or $i=\max$, these coefficients are obtained from the flux boundary conditions (BCs) discussed subsequently.

\subsubsection{The electrostatic field}

As explained in Chapter 6, the fluid equations are coupled to the electrostatic field via the simplified Poisson equation

$$
\varepsilon_{o} \nabla^{2} \mathrm{~V}=-\rho
$$

The charge density is determined from the number density of electrons and ions,

$$
\rho=e\left(n_{p}-n_{e}-n_{n}\right)
$$

To solve the Poisson equation, 1D integration must be taken along the axis of discharge.

$$
\int \nabla^{2} V(x) d x=-\frac{e}{\varepsilon_{o}} \int\left(n_{p}-n_{e}-n_{n}\right) d x
$$

Applying the divergence theorem and assuming constant charge density over the control volume 


$$
\frac{V_{i+1}-V_{i}}{\Delta x}-\frac{V_{i}-V_{i-1}}{\Delta x}=-\frac{e}{\varepsilon_{o}}\left(n_{p}-n_{e}-n_{n}\right) \Delta x
$$

This results in a 3 point form of Poisson

$$
2 V_{i}-V_{i+1}-V_{i-1}=\frac{\Delta x^{2} \cdot e \cdot\left(n_{p}-n_{e}-n_{n}\right)_{i}}{\varepsilon_{o}}
$$

Equation 7.27 can be solved via the TDMA

$$
\left[\begin{array}{ccccccc}
3-1 & 0 & 0 & & & 0 \\
-1 & 2 & -1 & 0 & \cdots & & 0 \\
0-1 & 2 & -1 & & & 0 \\
\vdots & & \ddots & & \vdots \\
0 & & \cdots & -1 & 3
\end{array}\right]\left[\begin{array}{c}
V_{1} \\
V_{2} \\
V_{3} \\
\vdots \\
V_{\max }
\end{array}\right]=\left[\begin{array}{c}
\frac{3}{4}\left(n_{p}-n_{e}-n_{n}\right)_{1} \\
\left(n_{p}-n_{e}-n_{n}\right)_{2} \\
\left(n_{p}-n_{e}-n_{n}\right)_{3} \\
\vdots \\
\frac{3}{4}\left(n_{p}-n_{e}-n_{n}\right)_{\max }
\end{array}\right] \frac{\Delta x^{2} e}{\varepsilon_{o}}+\left[\begin{array}{c}
2 V_{D} \\
0 \\
0 \\
\vdots \\
2 V_{G}
\end{array}\right]
$$

Potential discretization at the first and last nodes in Figure 7.3 is obtained by taking the Taylor series expansion of $f(x+\Delta x)$ and $f(x-\Delta x / 2)$ for the first node; and $f(x-\Delta x)$ and $f(x+\Delta x / 2)$ for the last node. This is required to impose the voltage BCs. Then the electric field at the electrode boundary $\left(\mathrm{E}_{\mathrm{BC}_{-} 1}\right.$ and $\mathrm{E}_{\mathrm{BC}_{-} 2}$ in Figure 7.3$)$ is obtained by assuming constant field gradient between the boundary and the adjacent faces.

\subsubsection{The boundary conditions (BCs)}

Besides constraining the model, BCs also couple the model to the SPDI parameters. For simplicity, oscilloscope data of voltage and discharge current are approximated as sinusoidal functions representing the plasma support phase in Chapter 6.

The voltage function represents a Dirichlet $\mathrm{BC}$, whereas the discharge current function represents a von Neumann BC. For the model in this chapter, the discharge current is computed by the voltage imposed from an external circuit solver. This discharge current reflects on the ionization, attachment and recombination collisions described previously. 


\subsubsection{The external circuit and voltage $B C$}

The transient response leading to breakdown is very sensitive to time-gradients of the voltage. Smith et al. (2011) increase the voltage via a parametric sweep until sparking; Koh and Park (2003) use a $50 \mathrm{kV}$ pulsed DC voltage of $1 \mu$ s duration and $1 \mathrm{~ns}$ rise-time to capture the ionization wave.

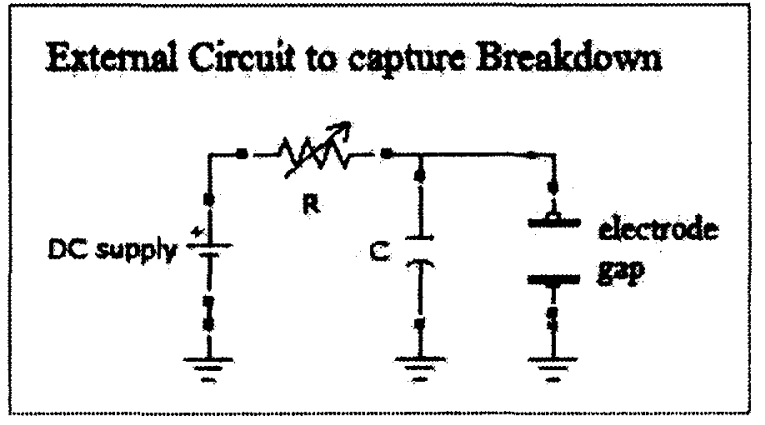

In this model, however, a simple external circuit shown in Figure 7.4 couples the circuit's potential to the discharge voltage, similarly to Farouk et al. (2006). This is important to ensure model stability.

Figure 7.4: The external circuit used in the model

From Figure 7.4 and elementary electronics theory, the discharge voltage is given by

$$
\frac{d V_{d}}{d t}+\frac{1}{R C} V_{d}=\frac{1}{R C}\left(V_{D C}-R I_{d}\right)
$$

where $V_{d}$ and $I_{d}$ are the discharge voltage and current respectively, $R$ is the resistor, $C$ is the capacitor, and $V_{D C}$ is the power supply voltage. The capacitance in the circuit controls the charging time. The power supply voltage must be larger than the breakdown voltage in air under the $1 \mathrm{D}$ approximation.

For the plasma support phase following breakdown, the gap is connected in series with a resistor and an AC supply. Hence the discharge voltage in this phase is given by

$$
V_{d}=\overline{V_{A C}} \cos \left(\omega_{S P D I} \cdot t\right)-R I_{d} .
$$

Here, the magnitude of the $\mathrm{AC}$ supply, $\overline{V_{A C}}$, is equivalent to the $\mathrm{DC}$ voltage after breakdown. 


\subsubsection{Flux BCs}

Most models ignore diffusion at the wall to simplify the mathematics. This causes some under-estimation of the electron density distribution in the discharge gap as shown by Hagelaar (2000). However, diffusion effects diminish with pressure, and simplified electron/ion flux BCs will be used for this model.

Due to polarity change, a switching function must be incorporated to determine the direction of the boundary flux. Let $\boldsymbol{u}$ be the unit vector toward the electrode surface and $\boldsymbol{\xi}$ a switch function, then

$$
\boldsymbol{\xi}=\begin{aligned}
& 1 \text { if } \boldsymbol{E} \cdot \boldsymbol{u} \geq 0 \\
& 0 \text { if } \boldsymbol{E} \cdot \boldsymbol{u}<0
\end{aligned}
$$

For positive and negative ions, the wall flux is given by

$$
\begin{gathered}
\boldsymbol{F}_{p}=\frac{1}{4} v_{t h_{-} p} \cdot \boldsymbol{u} n_{p}+\xi \mu_{p} E n_{p} \\
\boldsymbol{F}_{n}=(\xi-1) \mu_{n} E \cdot \boldsymbol{u} n_{n}
\end{gathered}
$$

Note that the negative ions have no thermal velocity near the wall. This condition is used here since negative ions are much less energetic than positive ions for overcoming the potential barrier near the electrodes (Lymberopoulos \& Economou, 1996).

For electrons, wall flux must take into account secondary electron emission,

$$
\boldsymbol{F}_{e}=\frac{1}{4} v_{t h_{e}} \cdot \boldsymbol{u} n_{e}+(\boldsymbol{\xi}-1) \mu_{e} E n_{e}-\boldsymbol{\xi} \gamma \boldsymbol{F}_{\boldsymbol{p}} .
$$

In Equations 7.31 through 7.33, the thermal velocity of the boundary flux is computed by

$$
v_{t h}=\sqrt{\frac{8 k T_{i o n}}{\pi m_{i o n}}} .
$$


For the secondary electron emission coefficient (see Equation 2.15), we use $\gamma=0.06$, which is the average for $\mathrm{N}_{2}{ }^{+}$and $\mathrm{O}_{2}{ }^{+}$upon collision with a cathode surface that has a work function of $5 \mathrm{eV}$ (Sakiyama, 2010); this work function is typical for engineering alloys. It should be noted that most plasma simulations assume $\gamma=0.1$, as it does not have a significant impact on most types of plasma.

For the electron mean energy, the wall flux of the mean energy is the summation of the thermal energy and the energy of secondary emission electrons entering the plasma ( $T$. Farouk, personal communication, 2012)

$$
\boldsymbol{F}_{\varepsilon}=5 / 2 k T_{e}\left(\frac{1}{4} \boldsymbol{v}_{t h_{e}} \cdot \boldsymbol{u} n_{e}\right)-\boldsymbol{\xi} e \Delta E_{e} \gamma \boldsymbol{F}_{\boldsymbol{p}}
$$

where $\Delta E_{e}$ is the energy of secondary emission electrons. In this model, this is assumed to be $5 \mathrm{eV}$, the equivalent of the work function used to determine $\gamma$.

\subsection{The Discretization Schemes}

As shown previously, the solution to the conservation equations depends on the relaxation parameter $f$ of the FVM. Whether an explicit or implicit scheme is used, the coupling to the electrostatic field must be explicit or implicit accordingly.

\subsubsection{Explicit formulation of particle conservation}

For $f=0$, discretization of Equations 7.1 through 7.3, and 7.5 is explicit. The density field can be easily obtained from Equation 7.22 since the velocity field is known. The coupling sequence is as following:

1) Determine the electrostatic field from known electron and ion densities 
2) Determine the electron energy

3) Determine the electron/ion and electron energy density at the next time-step

4) Repeat steps 1 through 3

The stability of this scheme is given by the von Neumann criteria, such that the CourantFriedrichs-Lewy (CFL) time-step is

$$
\Delta t_{C F L}<\frac{\Delta x}{\left|v_{e}\right|+{ }^{2 D_{e} / \Delta x}} .
$$

Hence the time-step is limited by the electron flux time through the discretized control volume. Furthermore, for accurate results, explicit coupling to the electrostatic field requires a time-step lower than the dielectric relaxation time,

$$
\Delta t<t_{D R}=\frac{\varepsilon_{o}}{e\left(\mu_{e} n_{e}+\mu_{p} n_{p}+\mu_{n} n_{n}\right)} .
$$

For typical glow discharge at atmospheric pressure, $t_{D R}$ can be on the order of ps, which imposes large computational time since SPDI has a period of $28 \mu \mathrm{s}$.

\subsubsection{Semi and fully-implicit formulations of particle conservation}

For $0<f \leq 1$, discretization of Equations 7.1 through 7.3, and 7.5 is semi- or fullyimplicit. Recall that the electrostatic field must be coupled semi or fully-implicitly accordingly. Semi and fully implicit schemes have shown to increase the time-step constraint by 2 to 3 orders of magnitude.

The fully-implicit case is difficult, as it requires simultaneous solution of the particle densities and the potential. A TDMA solution of the form $[A(x)][x]=[b(x)]$ is possible. In this case matrix $[\mathrm{A}(\mathrm{x})]$ is composed of the tridiagonal matrices for the potential, electrons, positive and negative ions, hence resulting in a $4 x$ larger matrix; $[b(x)]$ is the 
vector accounting for previous time-step transport and the additional implicit terms; and $[x]$ is the solution vector of the potential, electrons, positive and negative ion densities.

Difficulties arise with the fully implicit case since iterative solutions such as NewtonRaphson are required for convergence. This requires the Jacobian of $[A(x)][x]-[b(x)]$, which is difficult to determine because it requires gradients of drift-diffusion flux with respect to the potential and electron/ion density, and most transport and rate coefficients are tabulated. Newton-Raphson convergence was implemented by Farouk (2009).

The fully-implicit case was attempted by estimating the Jacobian via the secant method. However, it turned out that the Jacobian matrix is close to singular. This is most likely due to imposing all flux boundary conditions when discretizing the particle density. Hagelaar (2000), (2008) and Balcon (2007) implemented the simpler semi-implicit coupling. For the model developed here, the sequence of Hagelaar (2000) is used:

1) Solve the electrostatic field semi-implicitly (see derivation in APENDIX B)

2) Solve for electron/ion and electron energy density semi-implicitly

3) Iterate step 2) with reduced time-steps until changes in densities are less than $25 \%$ The above case is a weak form of coupling that limits the density gradients in time. This is why step 3) requires time-step to be reduced temporarily. Hagelaar (2000) illustrates that the semi-implicit coupling above creates instabilities in the energy equation if the source term is computed explicitly. To avoid instabilities, the energy exchange with the electric field is computed implicitly, and the collisional energy exchange is linearized in time with respect to the mean electron energy; this is derived in APPENDIX C. 


\subsubsection{Coupling to the external circuit solver}

The voltage of the plasma discharge model is imposed by the external circuits shown in Figure 7.4; this requires the gap discharge current, which is determined by summing the conduction and displacement currents,

$$
I_{d}=\bar{A}\left(J_{c}+\frac{\varepsilon_{o}}{d} \frac{d V_{d}}{d t}\right)
$$

where $\bar{A}$ is the average cross sectional area of discharge (assumed to be the area of the powered electrode in SPDI), $J_{c}$ is the conduction current density, $\varepsilon_{o}$ is the vacuum permittivity, and $d$ is the gap size.

The current density due to conduction is constant in time and given by

$$
J_{c}=\frac{e}{d} \int_{0}^{d}\left(F_{p}-F_{n}-F_{e}\right) d x
$$

where $e$ is the elementary charge, and $\left(\boldsymbol{F}_{\boldsymbol{p}}-\boldsymbol{F}_{\boldsymbol{n}}-\boldsymbol{F}_{e}\right)$ is the net flux of charged particles, including the positive ions, negative ions, and the electrons.

Discharge voltage, as computed by Equation 7.28 for breakdown is determined implicitly to avoid instabilities. The discretization is

$$
\begin{aligned}
V_{d}^{k+1}\left[\frac{1}{\Delta t}(1+\right. & \left.\left.\frac{\varepsilon_{o} \bar{A}}{C d}\right)+\frac{1}{R C}\right] \\
& =V_{d}^{k}\left[\frac{1}{\Delta t}\left(1+\frac{\varepsilon_{o} \bar{A}}{C d}\right)\right]+\frac{1}{C}\left[\frac{V_{D C}}{R}-J_{c}^{k+1} \bar{A}\right] .
\end{aligned}
$$

For the plasma support phase, the discharge voltage is imposed by Equation 7.29, which is discretized semi-implicitly. First, the voltage across the resistor is determined from

$$
V_{R}^{k}=\overline{V_{A C}} \cos \left(\omega_{S P D I} \cdot(t+\Delta t)^{k-1}\right)-\left[V_{d}^{k}+V_{R}^{k} \frac{d \Delta t}{R \bar{A} \varepsilon_{o}}\right] .
$$


The bracketed term on the RHS is the anticipated ${\widetilde{V_{d}}}^{k+1}$ due to the displacement current. If only the the explicit term $V_{d}^{k}$ is used, the model is numerically unstable.

The discharge voltage is then calculated from

$$
V_{d}^{k+1}=V_{d}^{k}+\left(V_{R}^{k}-R \bar{A} \int_{c}^{k}\right) \frac{d \Delta t}{R \bar{A} \varepsilon_{o}}
$$

\subsection{Summary of the overall solver}

Recall that breakdown is determined by the transient response of the discharge current. Quasi-neutrality is insignificant during this duration; hence the local field approximation is valid. On the other hand, the quasi-steady state discharge is governed by the balance of particle kinetics. If plasma is produced, quasi-neutrality forces ambipolar diffusion. The most important aspect of breakdown is the time-constraint required to capture the transient ionization waves. It was determined that semi-implicit coupling does not benefit breakdown's computational time, since temporarily reduced time-steps were the same order of magnitude as the time-step for explicit coupling.

The model uses the following scheme. An explicit solver using the LFA is used to determine the breakdown phase. The transport Equations 7.1 to 7.3 and the potential field Equation 7.23 are discretized in 1D using the FVM. Particle and electric field distributions immediately after breakdown are used as initial conditions in a semi-implicit solver, which also discretizes the transport of energy given by Equation 7.5. Figure 7.5 illustrates a flowchart summarizing the overall plasma solver. 


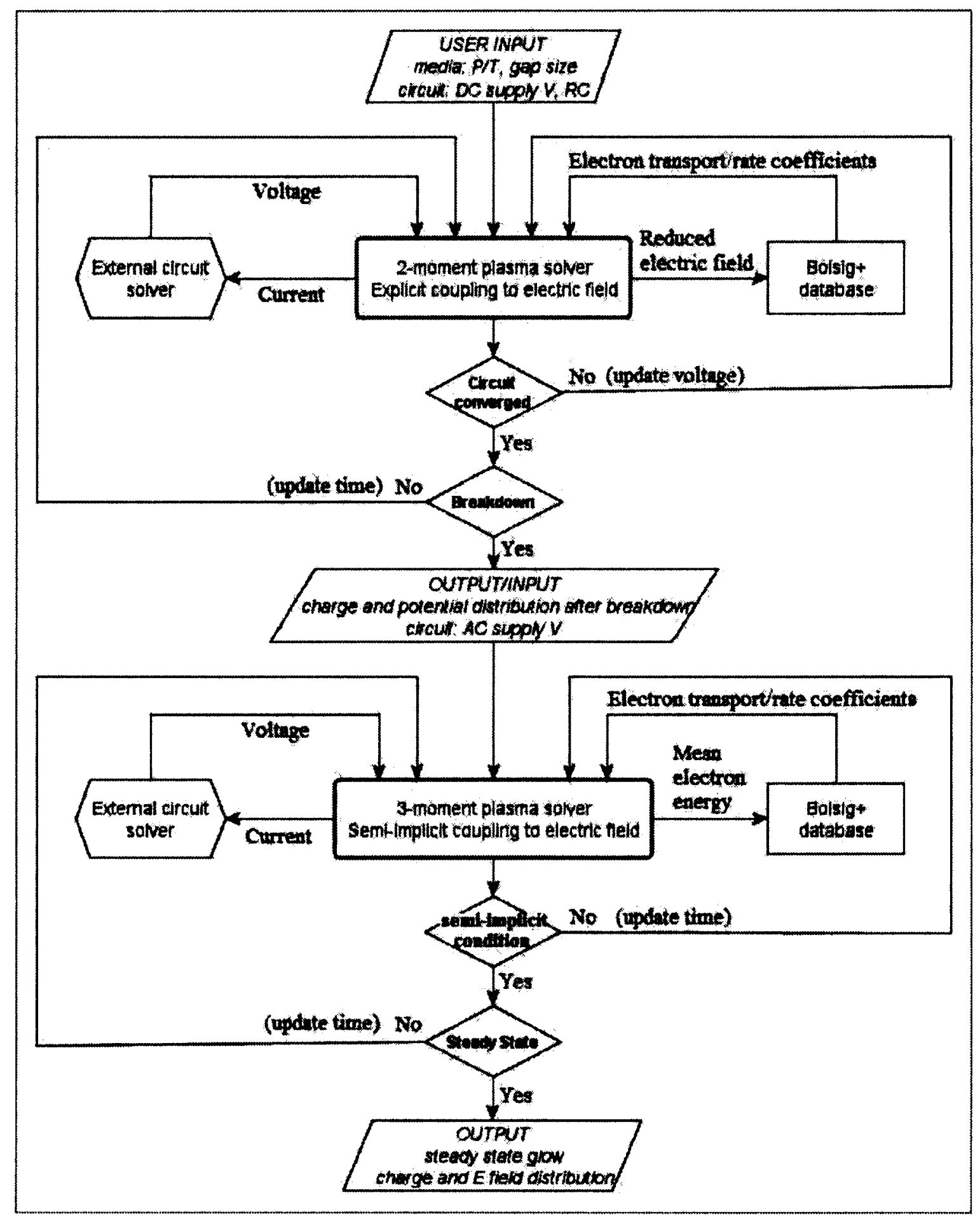

Figure 7.5: Flowchart of the overall plasma solver, implemented in Matlab 


\subsubsection{The link between the model and experimental SPDI discharges in air}

Recall that spark plug used for SPDI is a commercial spark-plug; hence powered electrode of diameter $\approx$ gap distance. For this geometry, breakdown voltage in air can be estimated empirically from (Mardiguian, 2009)

$$
V_{B}(k V)=3 P \cdot d+1.3 \sqrt{d}
$$

where the similarity parameter $P \cdot d$ is $a t m \cdot m m$. Hence the DC power supply of the external circuit in Figure 7.4 is set at

$$
V_{D C}=V_{B}(k V) .
$$

It should be noted that Equation 7.42 is only accurate for breakdown in the Townsend regime. 


\section{Chapter: ODE Interpretation of SPDI Discharge}

In this chapter, the plasma support phase of SPDI is analyzed via an ordinary differential equation (ODE). The purpose of the ODE model is to relate the charge in the sheath to the discharge transition observed experimentally.

The ODE model is based on the theoretical work by Czarnetzki et al. (2006) and Heil et. al (2008), which present a second order non-linear time-variant ODE known as plasma series resonance (PSR). In this thesis, there are 2 important additions to the ODE:

1) Ions, like electrons, are also carriers of electric current in the plasma bulk;

2) Space charge in the sheath is assumed to be a simulated function of the temporal sheath size.

The first addition to the ODE can be justified by comparing the ion - air molecule collision frequency with SPDI's driven frequency. From Equation 2.9, this collision frequency is given by

$$
\vartheta_{\text {in }}=\frac{e}{\mu_{i} m_{i}} .
$$

Assuming the average mass of $\mathrm{N}_{2}{ }^{+}, \mathrm{O}_{2}{ }^{+}$and $\mathrm{O}_{2}{ }^{-}$ions, and an average ion mobility of 3.11 $\mathrm{cm}^{2} / \mathrm{sV}$ in atmospheric air (Smith et al., 2011), then the ion - air molecule collision frequency is approximately $1.03 \mathrm{Mrad} / \mathrm{s}$. Recall from Chapter 6, that SPDI has a driving frequency of approximately $0.224 \mathrm{Mrad} / \mathrm{s}$, which is an order of magnitude lower. Thus ions can respond to the variations of the external electric field and may have significant contribution to the discharge current. 
The second addition couples the distributions of the plasma model in Chapter 7 to the $\mathrm{ODE}$, and is the fundamental assumption in this chapter. Specifically, space charge distribution near the electrodes is assumed to be a curve-fitted function of the sheath thickness; this introduces the spatial dependence of the sheath dynamics.

\subsection{Simple Interpretation of a plasma bounded by the electrodes}

Figure 8.1 below shows a schematic of the discharge gap. The excitation voltage $V_{P}$, and

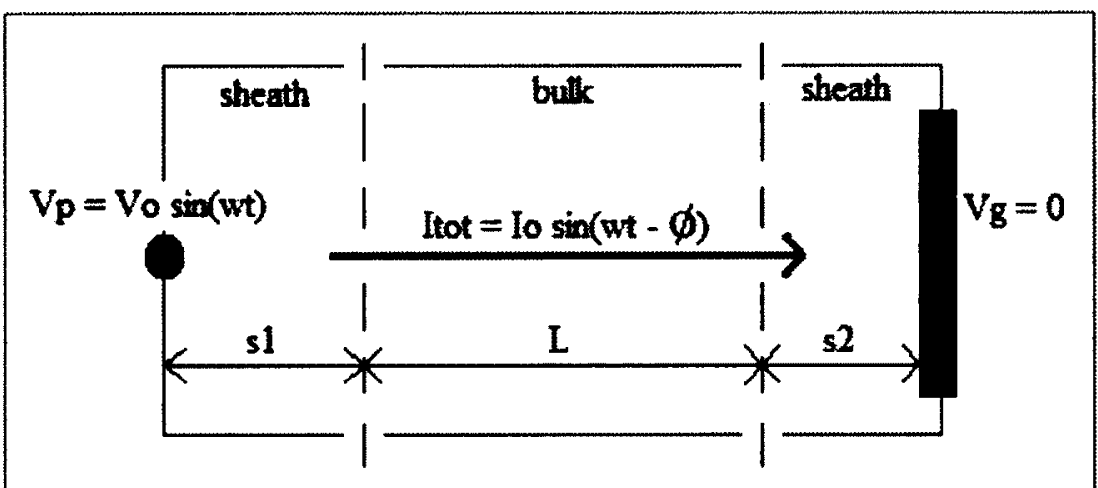

Figure 8.1: Schematic of the SPDI discharge the discharge current $I_{\text {tot, }}$ are the voltagecurrent waveforms of the plasma support phase. Sheath thickness at the

powered and ground electrodes is represented by $s_{1}$ and $s_{2}$ respectively, and $L$ is the plasma bulk thickness. This is a basic interpretation of a glow discharge, which is presented in Chapter 4.

\subsection{Assumptions and Derivations for deriving the ODE}

It is assumed that in the plasma bulk, most of the discharge current is conduction due to electron and ion momentum. Due to quasi-neutrality in this region, ionization is approximately constant (Heil et al., 2008). Displacement current is ignored since the excitation frequency $(35.7 \mathrm{kHz})$ is insignificant compared to electron and ion frequencies. Furthermore, if inertia, pressure gradients, and inelastic collisions are neglected and 
collision frequency is assumed constant, conservation of momentum given by Equation 4.6 simplifies to

$$
\frac{m}{e}\left(\frac{\partial}{\partial t}+\vartheta\right) \int_{s 1}^{L+s 1} v \cdot d s=V_{b u l k}
$$

where $V_{\text {bulk }}$ is the potential difference across the plasma bulk.

The path of integration is the direction of current flow. Then the plasma velocity integral is related to the total current by

$$
\int_{s 1}^{L+s 1} v \cdot d s=I_{t o t}(t) \frac{L}{\bar{n} \bar{A}}
$$

where $\bar{n}$ is average particle density due to electrons and ions contributions, and $\bar{A}$ is the average discharge cross section.

The average discharge cross section is assumed to be the area of the powered electrode (this is the same assumption used in Chapter 7). By plugging Equation 8.2 into 8.1, voltage across the plasma bulk becomes

$$
\begin{aligned}
\frac{L}{\pi r_{p}^{2} e^{2}}\left[\frac{m_{e}}{n_{e}}\left(\vartheta_{e n} I_{\text {tot }}(t)+\frac{\partial}{\partial t} I_{\text {tot }}(t)\right)\right. \\
+\frac{m_{n}}{n_{n}}\left(\vartheta_{\text {in }} I_{\text {tot }}(t)+\frac{\partial}{\partial t} I_{\text {tot }}(t)\right) \\
\left.-\frac{m_{p}}{n_{p}}\left(\vartheta_{\text {in }} I_{\text {tot }}(t)+\frac{\partial}{\partial t} I_{\text {tot }}(t)\right)\right]=V_{\text {bulk }}(t)
\end{aligned}
$$

where $r_{p}$ is the radius of the powered electrode. It should be noted that in Equation 8.3, collision frequencies of the electrons and ions with the air molecules are assumed constant.

Equation 8.3 can be simplified assuming $m_{p}=m_{n}$. This assumption is valid since the difference between the positive and negative ion mass is negligible. Let the following 
terms represent the electron and ion contribution to the plasma's "combined particle density" $n$ ", "combined mass" $m$ ", and "combined collision frequency" $g^{*}$, given by

$$
\begin{gathered}
n^{*}=n_{e} n_{n} n_{p} \\
m^{*}=m_{e} n_{n} n_{p}+m_{i o n} n_{e}\left(n_{p}-n_{n}\right) \\
\vartheta^{*}=\frac{\vartheta_{e n} \cdot m_{e} n_{n} n_{p}+\vartheta_{i n} \cdot m_{i o n} n_{e}\left(n_{p}-n_{n}\right)}{m^{*}}
\end{gathered}
$$

Equations 8.4 through 8.6 will be used to simplify Equation 8.3 .

Discharge current can be defined in terms of the time derivative of charge

$$
I_{\text {tot }}(t)=\frac{\partial Q}{\partial t}
$$

Then Equation 8.3 reduces to

$$
\frac{m^{*}}{n^{*} e^{2}} \frac{L}{\pi r_{p}^{2}}\left(\vartheta^{*} \frac{\partial Q(t)}{\partial t}+\frac{\partial^{2} Q(t)}{\partial t^{2}}\right)=V_{b u l k}(t)
$$

In the sheath, the electric field is composed of field due to conduction current and the displacement field. Heil et al.(2008) show that the sheath's conduction current can be estimated by

$$
I_{\text {sheath }}(x, t) \approx \frac{n_{e}(x, t)}{n_{\text {ion }}(x)} \cdot I_{\text {total }}(t) .
$$

For the case of glow discharge produced by SPDI, it is assumed that conduction current in the sheath is negligible due to high electron depletion. The displacement field is assumed to be given by the electrostatic approximation (Poisson equation).

In the electrostatic approximation, charge density in the sheath becomes

$$
\sigma(t)=e \int_{0}^{s(t)}\left(n_{p}-n_{n}-n_{e}\right) d x
$$


where $s(t)$ is the temporal sheath expansion.

Time variation of the sheath voltage can be written as

$$
\frac{d V_{s}}{d t}=\frac{\partial V_{s}}{\partial s} \frac{d s}{d t} .
$$

The first RHS term in Equation 8.11 can be replaced by Poisson equation

$$
\frac{\partial V_{s}}{\partial s}=-\frac{e}{\varepsilon_{o}}\left(n_{p}-n_{n}-n_{e}\right) \cdot s .
$$

Here, it is assumed that the net density (number density difference of positive and negative charges) is dependent on the sheath thickness as

$$
n_{n e t}(s)=N s^{M}
$$

where $N$ and $M$ are variable-units coefficients based on the electron and ion distributions in the sheath; $s$ is the sheath thickness.

Then the charge density in the sheath given by Equation 8.10 becomes a function of the sheath thickness

$$
\begin{gathered}
\sigma(t)=e N \frac{s(t)^{M+1}}{M+1} \\
s(t)=\sqrt[M+1]{\frac{(M+1) \sigma(t)}{e N}}
\end{gathered}
$$

By plugging Equations 8.14 and 8.15 into Equation 8.11 , the sheath voltage is expressed in terms of its charge density

$$
V_{s}=\left(-\frac{1}{\varepsilon_{o}} \sqrt[M+1]{\frac{M+1}{e N}}\right) \int_{0}^{\sigma(t)} \sigma^{1 / M+1} d \sigma .
$$

Assuming homogenous sheath charge across the electrode cross sectional area, $\sigma=\frac{Q}{A_{s}}$, 


$$
V_{s}=\left(-\frac{1}{\varepsilon_{o}} \sqrt[M+1]{\frac{M+1}{e N}} \frac{M+1}{M+2}\right)\left(\frac{Q}{A_{s}}\right)^{M+2 / M+1} .
$$

Equation 8.17 indicates that sheath voltage is inversely proportional to the electrode surface area. Since the cross sectional area of the powered electrode is much smaller than the cross sectional area of the ground electrode (see Chapter 6), $A_{s}=\pi r_{p}^{2}$.

\subsection{Dimensional form of ODE}

The ODE is developed by adding the sheath and plasma bulk contributions to the discharge voltage. The sum of Equations 8.8 and 8.17 represents the discharge voltage

$$
\begin{aligned}
V(t)=\frac{m^{*}}{n^{*} e^{2}} & \frac{L}{\pi r_{p}^{2}}\left(v^{*} \frac{\partial Q(t)}{\partial t}+\frac{\partial^{2} Q(t)}{\partial t^{2}}\right) \\
& -\left(\frac{1}{\varepsilon_{o}} \sqrt{\frac{M+1}{e N}} \frac{M+1}{M+2}\right)\left[\left(\frac{Q(t)}{A_{s}}\right)^{M+2 / M+1}\right]
\end{aligned}
$$

where the forcing potential function

$$
V(t)=V_{o} \sin (w t-\phi)
$$

and the discharge current function

$$
I_{\text {tot }}(t)=I_{o} \sin (w t)
$$

represent the plasma support phase illustrated in Chapter 6. Note that here, the discharge current is referred to as $I_{\text {tot }}(t)$. The capacitive phase shift is represented by $\phi$.

\subsubsection{Non-dimensional form of ODE}

Equation 8.18 is more practical when non-dimensionalized. Using the non-dimensional coefficients for charge, time, driven frequency, and collision frequency,

$$
q=\frac{Q}{Q_{o}}
$$




$$
\begin{aligned}
& \tau=\omega_{0} t \\
& \Omega=\frac{\omega}{\omega_{0}} \\
& \kappa=\frac{v^{*}}{\omega_{0}}
\end{aligned}
$$

Simple derivations give the following reference parameters

$$
\begin{gathered}
Q_{o}=A_{s}\left(\varepsilon_{o} \sqrt{\frac{e N}{M+1} \frac{M+2}{M+1} V_{o}}\right)^{M+1 / M+2} \\
\omega_{o}=\sqrt{\frac{n^{*} e^{2} \pi r_{p}^{2} V_{o}}{m^{*} L Q_{o}}}
\end{gathered}
$$

Then the non-dimensional ODE equation is given by

$$
\ddot{q}+\kappa \dot{q}-q^{M+2 / M+1}=\sin (\Omega \tau-\phi) \text {. }
$$

\subsubsection{Analogy to other systems}

Equation 8.27 is analogous to a mass-damper-spring system with time-varying external force. The charge in the gap represents mass, the collision frequency represents damping, and displacement field in the sheath represents the spring. Similarly to potential - kinetic energy transfer in the mass-damper-spring system, Equation 8.18 exhibits electrostatic magnetic transfer of energy. However, due to non-linearity of charge in the sheath, the ODE's analysis is challenging.

\subsubsection{Plasma series resonance represented by the ODE}

Plasma produced in the discharge can be analyzed as a series RLC circuit, where the plasma bulk characterizes the circuit's resistance and inductance, and the sheath characterizes the circuit's non-linear capacitance. This implies no resistive energy 
dissipation in the sheath, which is assumed valid due to negligible sheath thickness in high pressure glow discharges. It should be noted that two heating mechanisms occur inside the plasma sheath: Joule heating due to electron/ion acceleration in the electrostatic field, and stochastic heating due to temporary inhomogeneous electric fields. The causes of stochastic heating are still being researched.

Plasma's inductance and resistance are shown explicitly in Equation 8.18

$$
\begin{gathered}
\mathcal{L}=\frac{m^{*}}{n^{*} e^{2}} \frac{L}{\pi r_{p}^{2}}=\frac{V_{o}}{Q_{o} \omega_{o}^{2}} \\
\mathcal{R}=\nu^{*} \mathcal{L}=\frac{\kappa V_{o}}{Q_{0} \omega_{o}}
\end{gathered}
$$

The sheath capacitance does not have an explicit form. As shown by Equation 8.15, the sheath's periodic expansion and contraction length is expressed in terms of its instantaneous charge.

\subsection{Analysis of the ODE}

Linear control theory can be used to linearize non-linear ODEs around equilibrium point(s) using the first order Taylor series. However, due to the time-dependence of the forcing input, the ODE derived in this chapter does not have an equilibrium point. This is easily observed in state-space representation.

Let $x_{1}=q, x_{2}=\dot{x}_{1}$ and $u(\tau)=\sin (\Omega \tau-\phi) ;$ then Equation 8.27 can be represented

$$
\left[\begin{array}{l}
\dot{x_{1}} \\
\dot{x_{2}}
\end{array}\right]=\left[\begin{array}{c}
x_{2} \\
-\kappa x_{2}+x_{1}{ }^{M+2} / M+1+u(\tau)
\end{array}\right]
$$

This is a non-autonomous system $\dot{x}=f(x, u(\tau))$; the equilibrium point is defined at $x_{0}$ such that $f\left(x_{0}, \tau\right) \equiv 0 \quad \forall \tau \geq \tau_{o}$ (Slotine \& Li, 1991). However, the solution at $\dot{x}=0$ is 


$$
x=\sqrt[M+2 / M+1]{u(\tau)}
$$

Thus no equilibrium points exist.

\subsubsection{Non-linear system analysis}

The parameter-plane analysis described by Moore and Siljak (1967) will be used to analyze Equation 8.27. This method implies that steady solutions exist, which is similar concept to frequency response in linear systems. The key component in this analysis is that the non-linearity can be harmonically linearized using the describing function method, which is explained by Slotine and $\mathrm{Li}(1991)$. This analysis is appropriate for the ODE derived in this chapter since the electrode potential is known, and the discharge current and voltage in the plasma support phase have equivalent frequency.

\subsection{Summary of the ODE's Purpose}

The purpose of ODE is to analyze the charge in the plasma sheath, characterized by the non-linearity of Equation 8.27 . We postulate that the discharge transition observed experimentally can be explained by the magnitude of this non-linearity. 


\section{Chapter: Results and Discussion of the Plasma Model and the ODE}

Simulation results of the 1D plasma model described in Chapter 7 and the ODE equation derived in Chapter 8 are presented and discussed in this chapter. The electric field and plasma distributions are validated with theories of electric discharges presented in Chapter 4. The ODE's nonlinearity is related to the experimentally observed discharge transition.

\subsection{Results of the Plasma Model}

The following results are based on a 200 nodes linear mesh. This was found sufficient to ensure that numerical diffusion does not affect the accuracy of the results.

\subsubsection{Transient plasma during breakdown}

Simulations were initiated by assuming a low and uniform degree of ionization of $10^{-14}$ throughout the gap, and a potential difference of $10 \mathrm{~V}$. Voltage of the DC supply is given by Equation 7.42 .

The time-step was found to be highly sensitive to pressure and RC of the external circuit (recall that to first approximation, particle collision frequency scales with pressure, which explains the time-step reduction). Hence an adaptive time-step was used, where

$$
\Delta t=\min \left(\Delta t_{S I M}, \frac{1}{2} \Delta t_{D R}, \frac{1}{2} \Delta t_{C F L}\right)
$$

The adaptive time-step ensures that a small enough time-step is used during initiation, while maintaining stability; $\Delta t_{S I M}$ scales inversely with pressure. Low, medium, and high air pressure (relative to SPDI) breakdown is presented. The RC time constant was set at 1 ns, and the resistance was set at $1 \mathrm{M} \Omega$. 


\subsubsection{Breakdown at low-pressure (1 atm)}

Space charge and electric field distributions for atmospheric pressure breakdown are shown in Figure 9.1a. Also, space averaged electron density and the discharge voltage current are shown in Figure 9.1b.

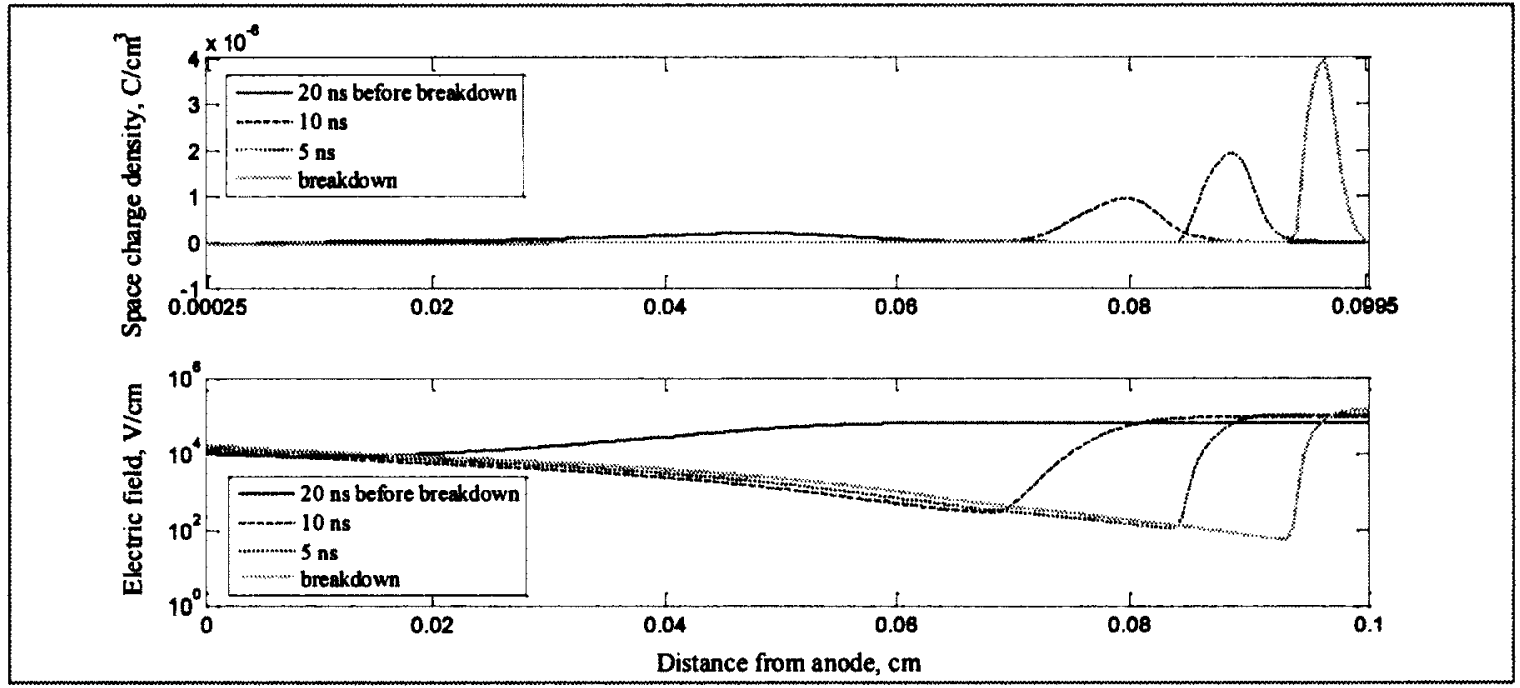

Figure 9.1a: Space charge and electric field distributions for breakdown at 1 atm
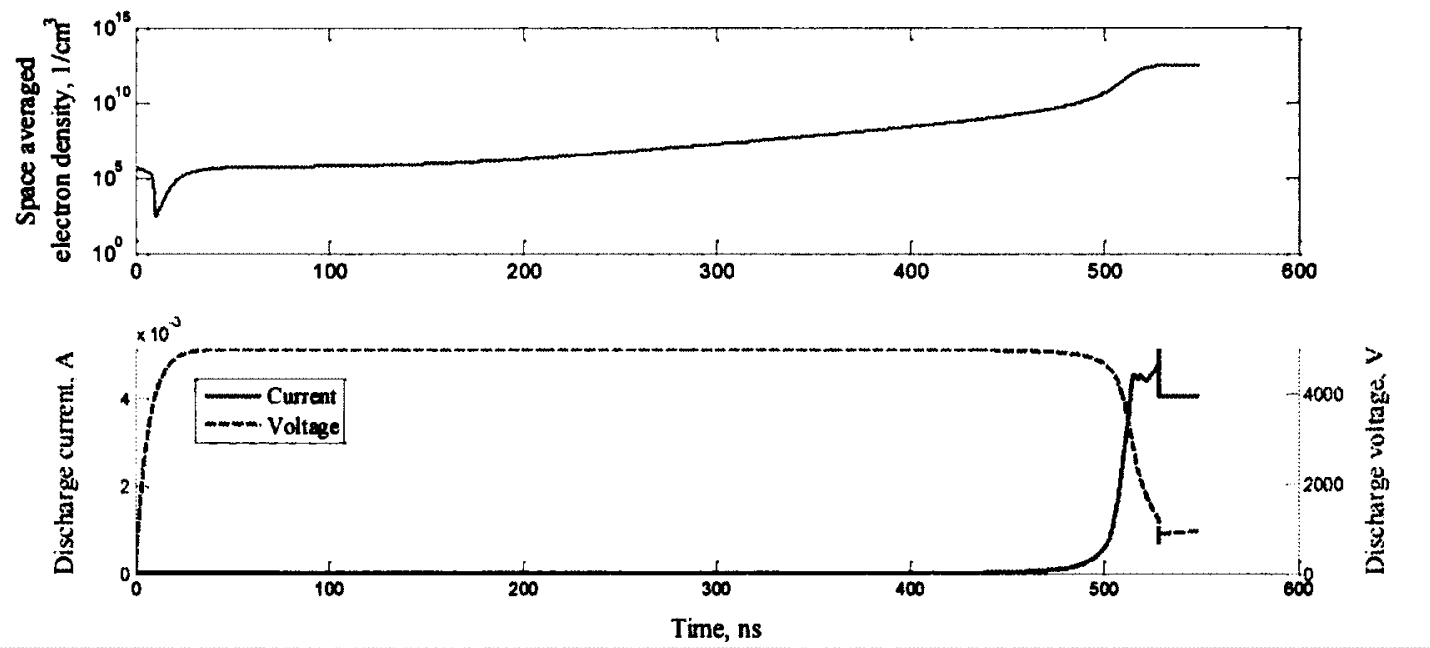

Figure 9.1b: Space average electron density and discharge voltage-current for breakdown at 1 atm 
Figure 9.1 a illustrates the propagation of the ionization wave during the $20 \mathrm{~ns}$ prior to breakdown (note that in this chapter, breakdown is assumed to be the instant where the discharge current peaks). It can be observed that the local space charge causes a rise of the electric field slightly ahead of the ionization front. The ionization wave velocity at breakdown is estimated by computing the time between the consecutive space charge fronts. At $1 \mathrm{~atm}$, velocity of the ionization wave is approximately $1.6 \cdot 10^{6} \mathrm{~cm} / \mathrm{s}$.

At breakdown, the space-averaged electron density reaches $3.9 \cdot 10^{12} \mathrm{~cm}^{-3}$, while the discharge current grows exponentially to $5.15 \mathrm{~mA}$; this is illustrated in Figure $9.1 \mathrm{~b}$. Immediately after breakdown, the voltage and current begin to stabilize and the DC glow discharge appears, in which the large cathode fall (high electric field) is essential. The model was also tested with lower resistances, and the discharge power increased accordingly.

In order to verify the code, the atmospheric pressure breakdown was tested with linear meshes of 50, 100, 200 and 250 nodes. Figure 9.2 illustrates the breakdown current, and time and space averaged electron density for different number of nodes; $\left|n_{e}\right|$ obtained via

$$
\left|n_{e}\right|=1 / d / t_{b r} \int_{0}^{t_{b r}} \int_{0}^{d} n_{e} d x d t
$$

where $d$ is the gap size, and $t_{b r}$ is the breakdown time. 


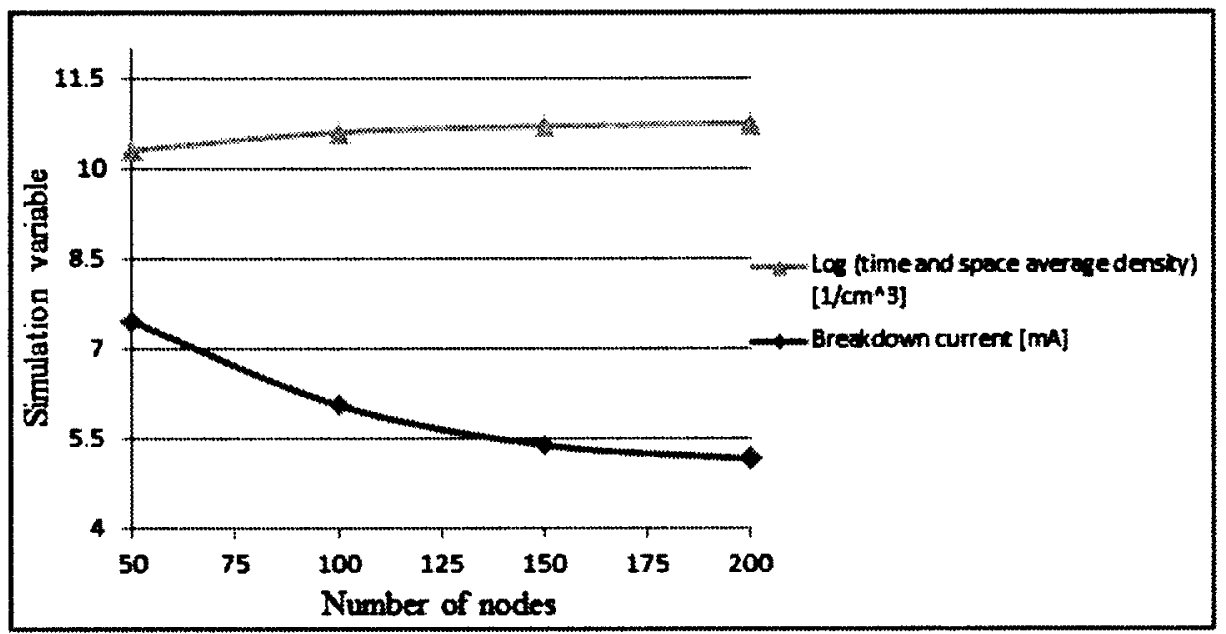

Figure 9.2: Breakdown current, and time and space averaged electron density for increasing number of nodes

Figure 9.2 indicates that time and space averaged electron density, and breakdown current are converging with increasing the number of nodes. This verifies the transient simulation.

\subsubsection{Breakdown at mid-pressure (5 atm)}

Space charge and electric field distributions of breakdown at 5 atm are shown in Figure 9.3a. Also, space-averaged electron density and discharge voltage - current are shown in Figure 9.3b. 


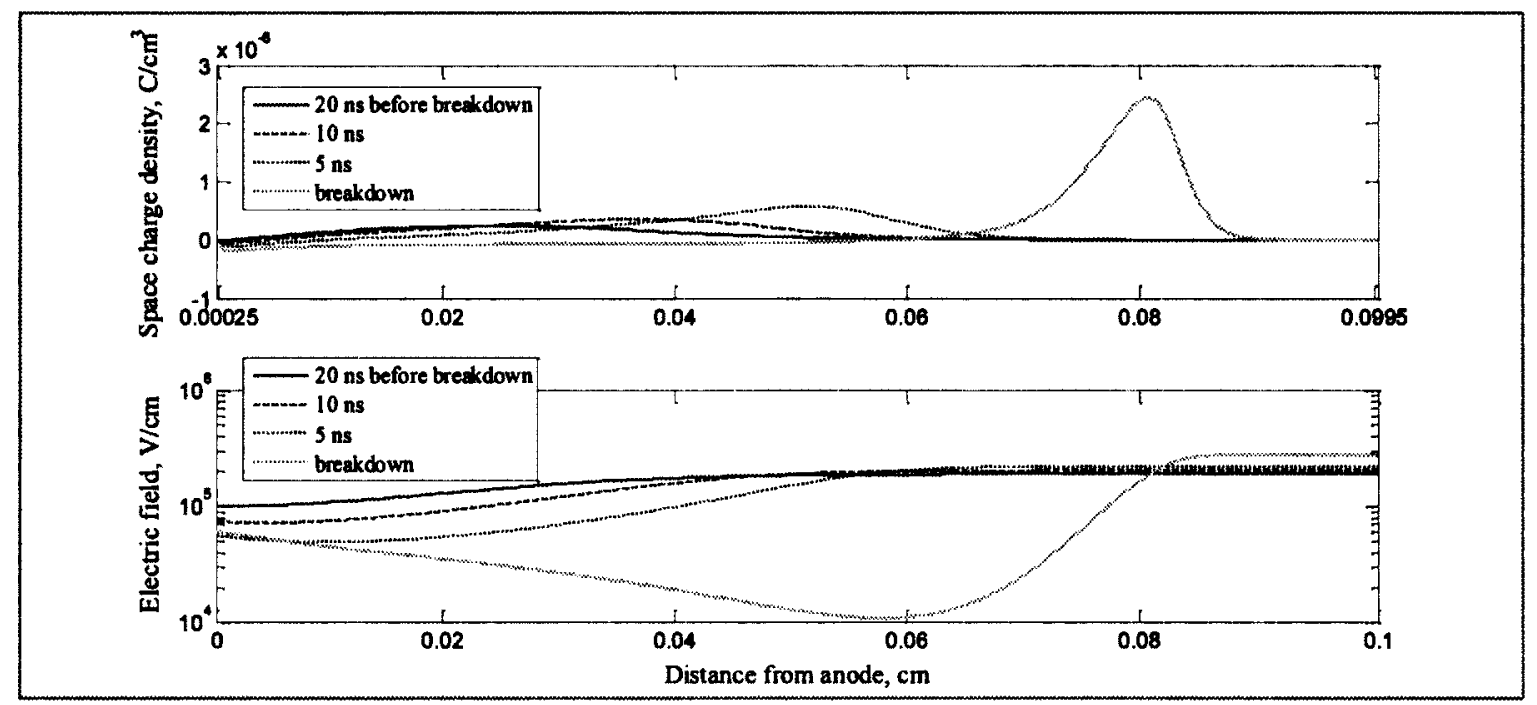

Figure 9.3a: Space charge and electric field distributions for breakdown at $5 \mathrm{~atm}$

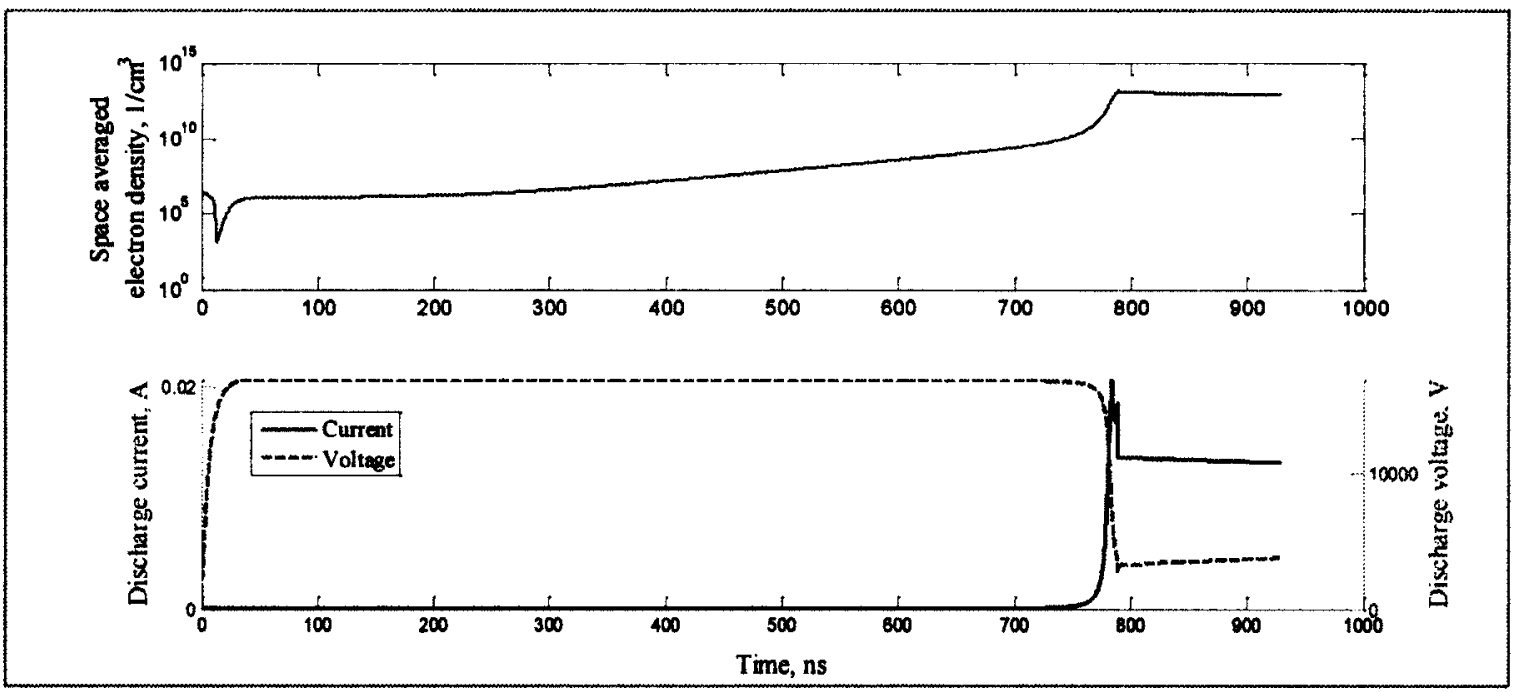

Figure 9.3b: Space average electron density and discharge voltage-current for breakdown at 5 atm

Similarly to breakdown at atmospheric pressure, transient space charge and electric field distributions during breakdown at 5 atm display rise of the electric field ahead of the ionization front. However, the ionization front does not reach the cathode at breakdown, as shown in Figure 9.3a. Ionization wave velocity at breakdown is approximately $5.8 \cdot 10^{6}$ 
$\mathrm{cm} / \mathrm{s}$, which is 4 times faster than the ionization velocity at atmospheric pressure breakdown.

Space average electron density for 5 atm breakdown reaches $1.2 \cdot 10^{13} \mathrm{~cm}^{-3}$, while the discharge current grows exponentially to $20.65 \mathrm{~mA}$ as shown in Figure $9.3 \mathrm{~b}$. It should be noted that the discharge power after breakdown is significantly higher at 5 atm compared to atmospheric pressure. Hence higher power DC discharges can be sustained if pressure is increased.

\subsubsection{Breakdown at high-pressure (14 atm)}

Space charge and electric field distributions of breakdown at 14 atmospheres are shown in Figure 9.4a. Also, space averaged electron density and discharge voltage - current are shown in Figure 9.4b.

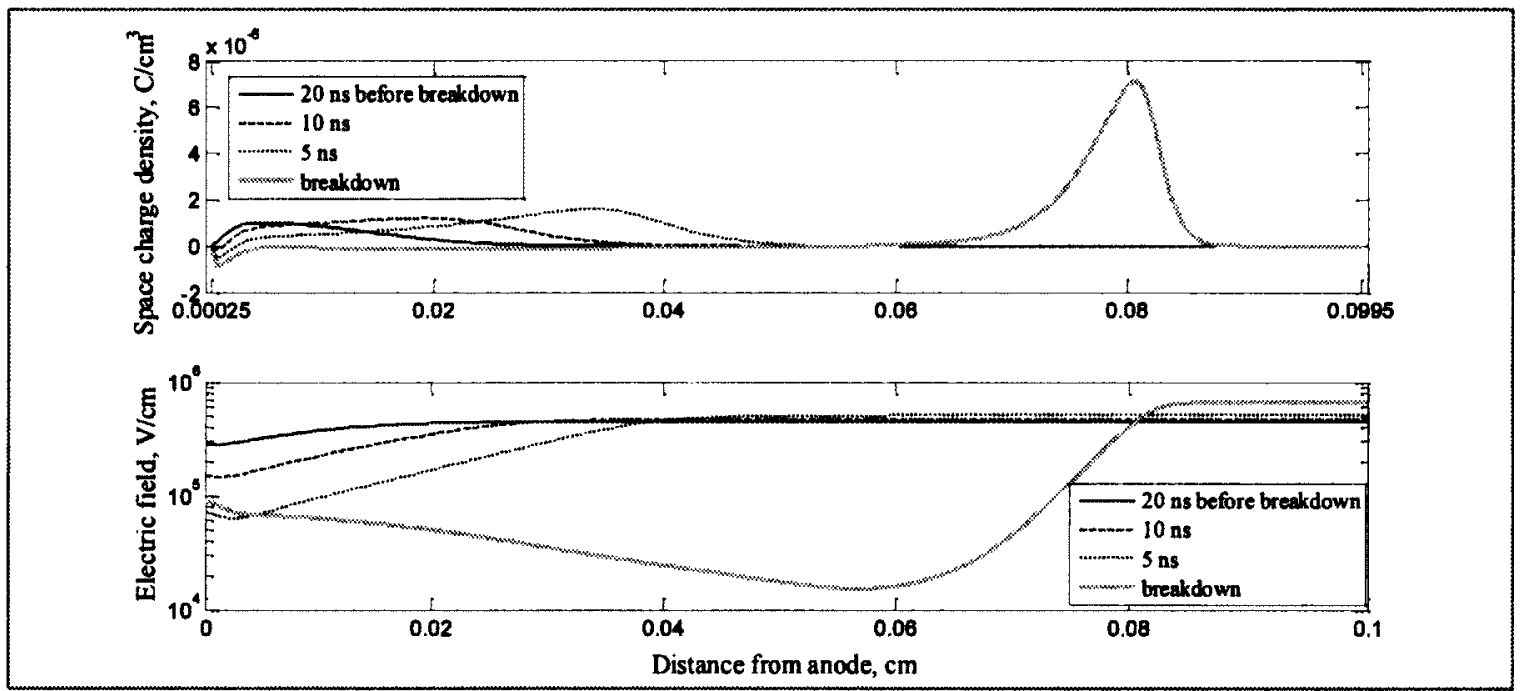

Figure 9.4a: Space charge and electric field distributions for breakdown at 14 atm 
Figure 9.4a illustrates highly distorted electric field immediately prior breakdown. At 14 atm, the ionization velocity is approximately $9.4 \cdot 10^{6} \mathrm{~cm} / \mathrm{s}$, which is an order of magnitude higher than breakdowns at 1 and $5 \mathrm{~atm}$. It should be noted that in this case, breakdown occurs on an order of magnitude smaller time-scale (compare Figure 9.4b with Figures $9.1 \mathrm{~b}$ and $9.3 \mathrm{~b}$ ). Clearly, a high pressure medium facilitates the sparking due to streamer breakdown that is observed experimentally. Hence, the mechanism of breakdown should incorporate an accurate model of the photo-ionization source.

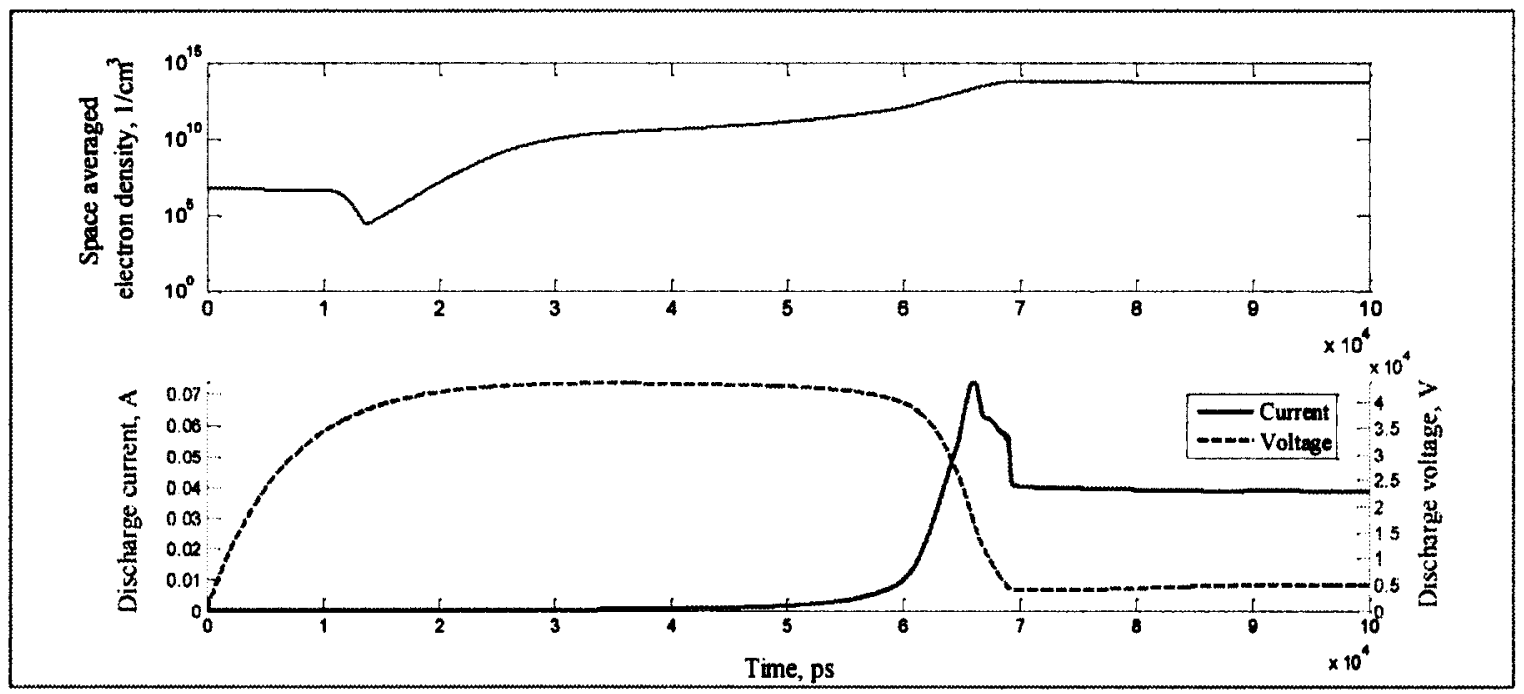

Figure 9.4b: Space average electron density and discharge voltage-current for breakdown at $14 \mathrm{~atm}$

This 1D plasma model predicts a space-averaged electron density of $5.9 \cdot 10^{13} \mathrm{~cm}^{-3}$, and discharge current of $74.47 \mathrm{~mA}$ for breakdown at $14 \mathrm{~atm}$, as shown in Figure 9.4b. It should be noted that experimental observations of SPDI postulate streamer discharges at this pressure (see Chapter 6). Hence the simplified mechanism in this model is not an accurate description of high pressure breakdown since it does not account for the photoionization terms accurately. 


\subsubsection{Parameters of breakdown tests}

A summary of the breakdown parameters for the cases above is provided in Table 9.1.

The rise of the ionization wave velocity at high pressure indicates that the source of photo-ionization must be computed accurately to improve the model predictions (see APPENDIX A).

Table 9.1: Summary of results for the breakdown simulations

\begin{tabular}{|l|l|l|l|l|l|}
\hline $\begin{array}{l}\text { Pressure, } \\
{[\mathrm{atm}]}\end{array}$ & $\begin{array}{l}\Delta t_{\text {SIM }}, \\
{[\mathrm{ps}]}\end{array}$ & $\begin{array}{l}\text { Breakdown } \\
\text { voltage, }[\mathrm{kV}]\end{array}$ & $\begin{array}{l}\text { Breakdown } \\
\text { current, }[\mathrm{mA}]\end{array}$ & $\begin{array}{l}\text { Ionization wave } \\
\text { velocity, }[\mathrm{cm} / \mathrm{s}]\end{array}$ & $\begin{array}{l}\text { Voltage after } \\
\text { breakdown, }[\mathrm{kV}]\end{array}$ \\
\hline 1 & 10 & 5 & 5.15 & $1.6 \cdot 10^{6}$ & 1.1 \\
\hline 5 & 8 & 17 & 20.65 & $5.8 \cdot 10^{6}$ & 3.8 \\
\hline 14 & 2 & 44 & 74.47 & $9.4 \cdot 10^{6}$ & 5.0 \\
\hline
\end{tabular}

It should be noted that the transition between the 2 modes of discharge in SPDI occurs around $8.5 \mathrm{~atm}$, which lies between the second and third pressure in Table 9.1. The following subsection discusses SPDI's transition from the point of view of breakdown.

\subsubsection{Relation between the ionization wave velocity and pressure}

As Table 9.1 indicates, the breakdown current and ionization wave velocity increase an order of magnitude near the experimentally observed transition pressure. Experimental data of the discharge voltage and current, and theories of Townsend and streamer breakdown validate these observations.

Here, we predict that based on the type of breakdown in SPDI, the plasma support phase is able to maintain a glow discharge. Specifically, the transition from Townsend to streamer breakdown determines the types of discharge in SPDI's plasma support phase. 


\subsubsection{Plasma distribution of RF and DC discharges}

Due to computational resource limitations, quasi-steady distributions were not obtained.

The duration for quasi-steady distributions of plasma in air is limited by the ion diffusion time. This can be approximated from

$$
t_{D I F U S E}=\frac{d^{2}}{2 D_{i}}
$$

where $d$ is the gap length $(0.1 \mathrm{~cm})$, and $D_{i}$ is the ion diffusivity in air, which is on the order of $10^{-2} \mathrm{~cm}^{2} / \mathrm{s}$ (Smith et. al, 2011). Hence the simulation would require running for a period on the order of $10^{-1} \mathrm{~s}$; this requires approximately $10^{9}$ time-steps. This is the reason most quasi-steady state simulations are for atomic gases such as Argon or Helium, where the ion diffusivity is 4 to 5 orders of magnitude larger.

It should be noted that the initial time-step, $\Delta t_{I N I}$, can be on the order of nanoseconds with the semi-implicit scheme, which reduces computational time considerably.

\subsubsection{Simulated RF discharges at atmospheric pressure}

The following results are transient plasma distributions at $13.56 \mathrm{MHz}$ and $1 \mathrm{~atm}$ pressure, averaged over the last $R F$ cycle. The magnitude of the $R F$ supply is equivalent to the discharge voltage immediately after breakdown $(1110 \mathrm{~V}$ for atmospheric pressure). The resistance of the external circuit is set to $100 \Omega$, which is equivalent to the resistance used to measure the discharge current in SPDI's plasma support phase. 


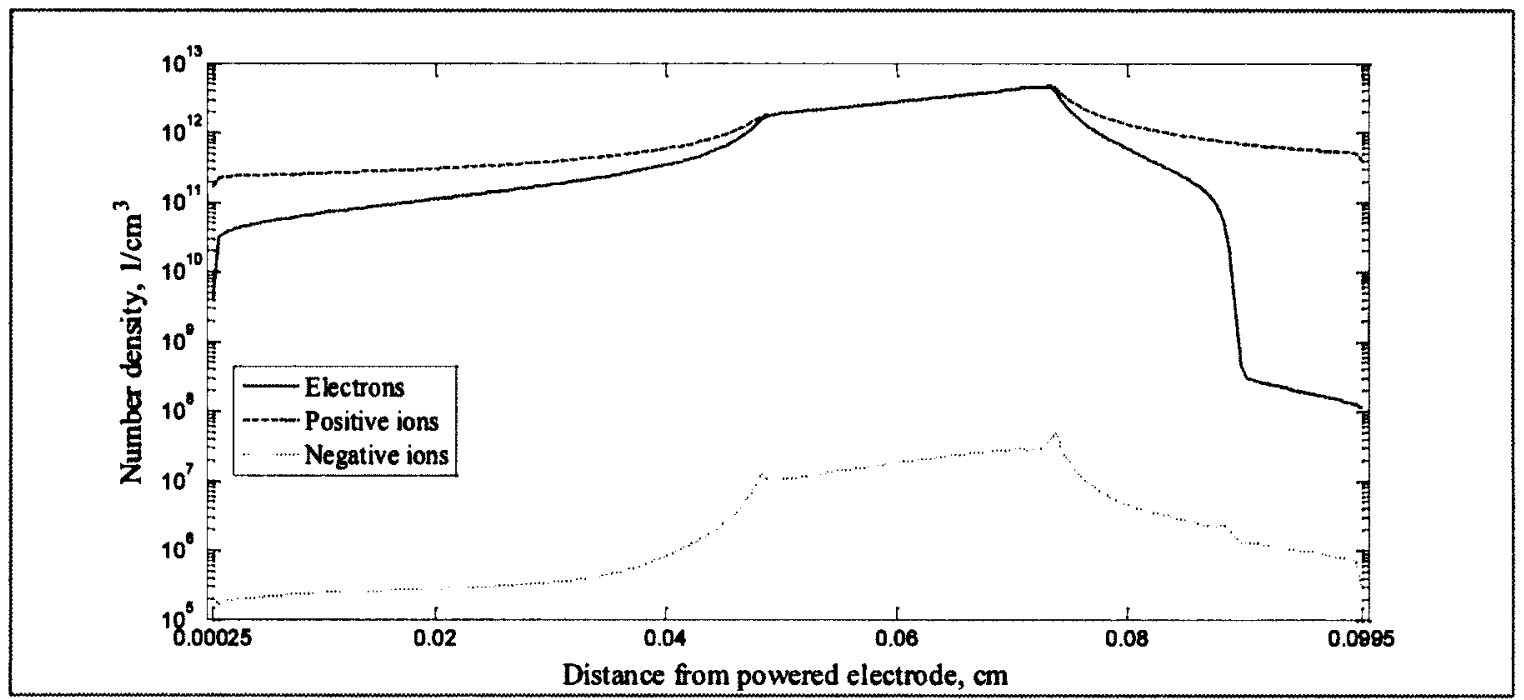

Figure 9.5a: Number density of species of the RF discharge at $1 \mathrm{~atm}$, averaged over the 10th cycle

Figure 9.5a illustrates the number density of electrons and ions averaged over the $10^{\text {th }}$ cycle at $13.56 \mathrm{MHz}$; this occurs around $0.7 \mu \mathrm{s}$. It can be observed that most of the gap is covered by the two positive sheaths near the electrodes (notice that the positive ion density is significantly higher in the sheath). The plasma density reaches $10^{12} \mathrm{~cm}^{-3}$ and its quasi-neutral region (around the center of the gap) is approximately $25 \%$ of the gap size. In quasi-steady state RF discharges in Argon at same frequency, plasma density reaches $10^{11} \mathrm{~cm}^{-3}$ and its quasi-neutral region is approximately $90 \%$ of the gap size (Balcon, 2007) and (Farouk, 2009). 


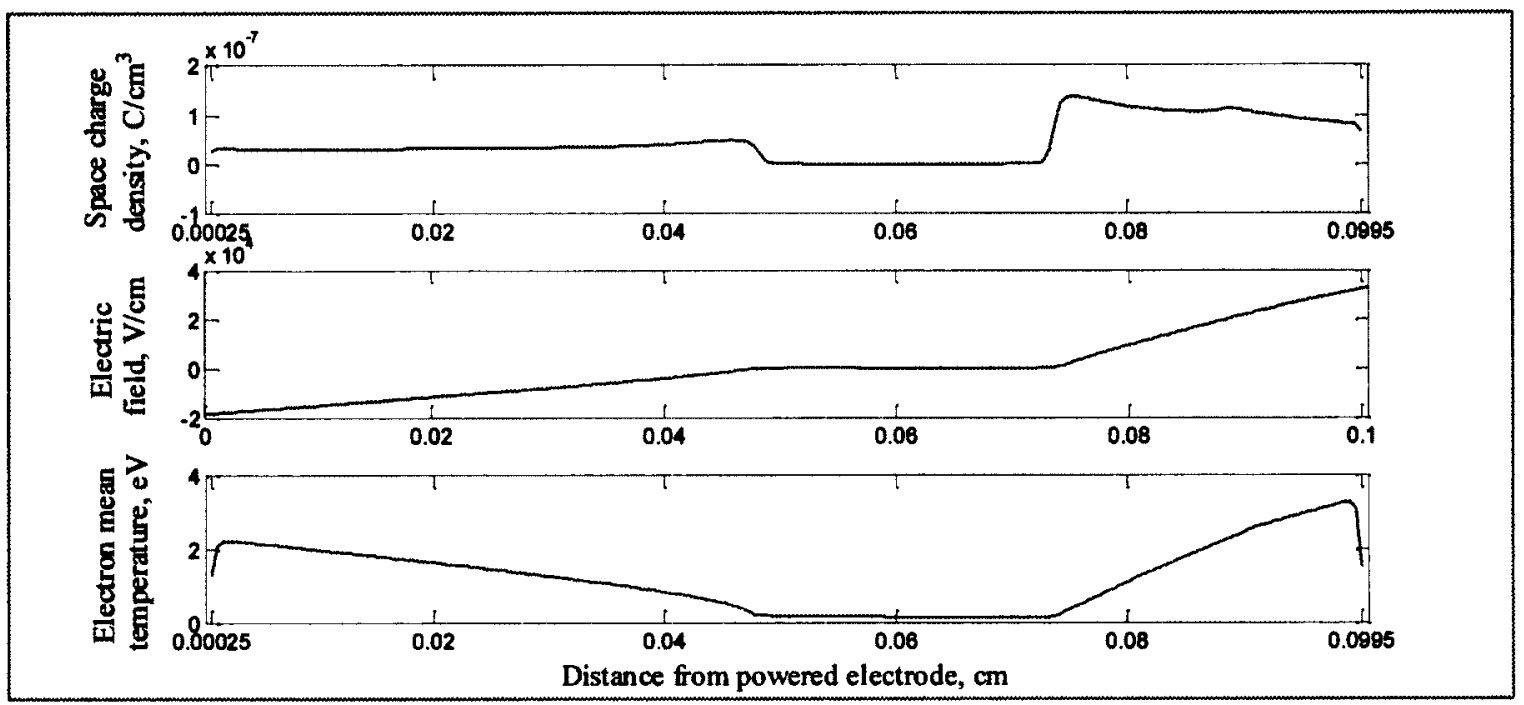

Figure 9.5b: Spatial space charge, electric field, and electron temperature of the RF discharge at 1 atm, averaged over the 10th cycle

As Figure 9.5b illustrates, the temporary quasi-neutral plasma region is sustained in very low electric fields (order of $1 \mathrm{~V} / \mathrm{cm}$ ), and electron temperatures of approximately $0.2 \mathrm{eV}$. However, the magnitude of the electric field near the electrodes peaks on the order of $10^{4}$ $\mathrm{V} / \mathrm{cm}$ in the sheaths, where the electron temperature reaches a temperature of 2 to $3 \mathrm{eV}$.

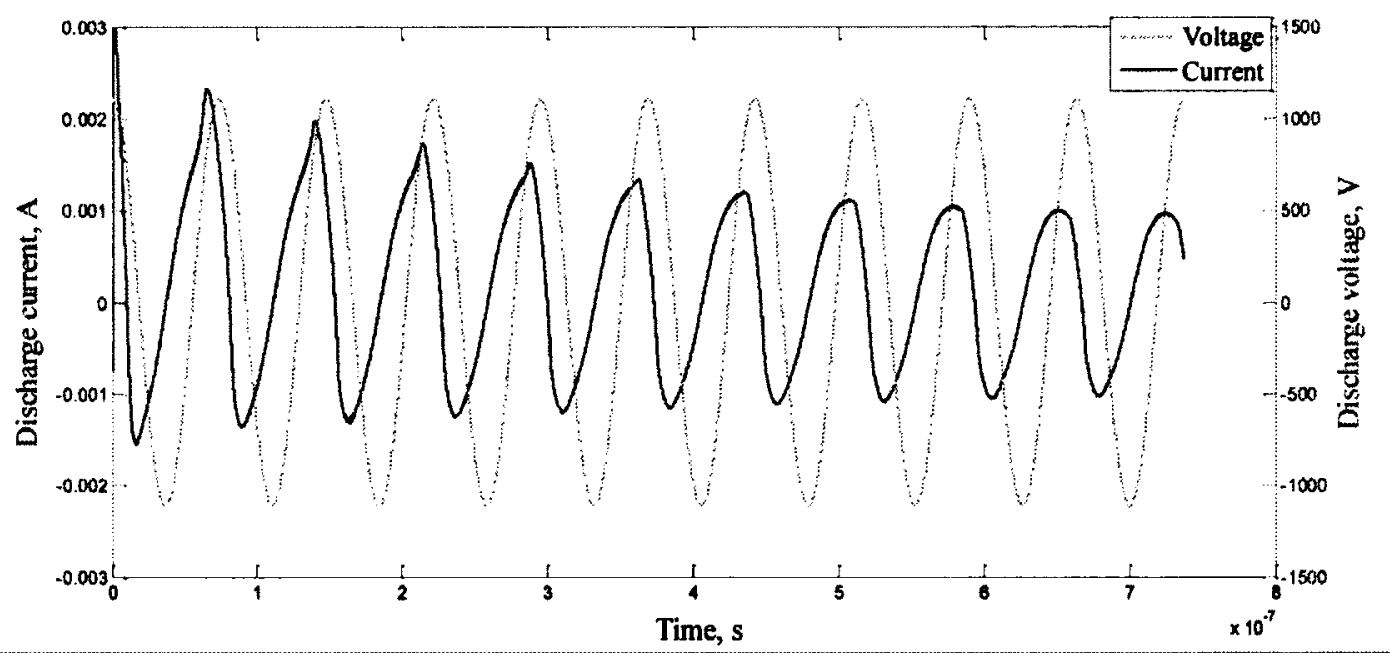

Figure 9.5c: Discharge voltage and current of the RF discharge at 1 atm, approaching the 10 th cycle 
Figure 9.5c indicates that the discharge voltage and current are beginning to stabilize; however the number of simulated cycles is very low at this point. It should be noted that the voltage-current waveforms in Figure 9.5c do not indicate a low power $\alpha$-mode $\mathrm{RF}$ glow discharge, which are presented in Balcon (2007) and Farouk (2009). This is due to the very low magnitude of the discharge current (around $1 \mathrm{~mA}$ ) and the spatially shifted electron densities near the electrodes.

Figure 9.6a illustrates the number density of electrons and ions averaged over the $100^{\text {th }}$ $\mathrm{RF}$ cycle at $13.56 \mathrm{MHz}$; this occurs around $7 \mu \mathrm{s}$. The electron and ion distributions indicate a nearly quasi-steady discharge, where the entire gap is uniformly and positively charged. However, the electron and positive ions densities drop to $10^{6}$ and $10^{10} \mathrm{~cm}^{-3}$ respectively.

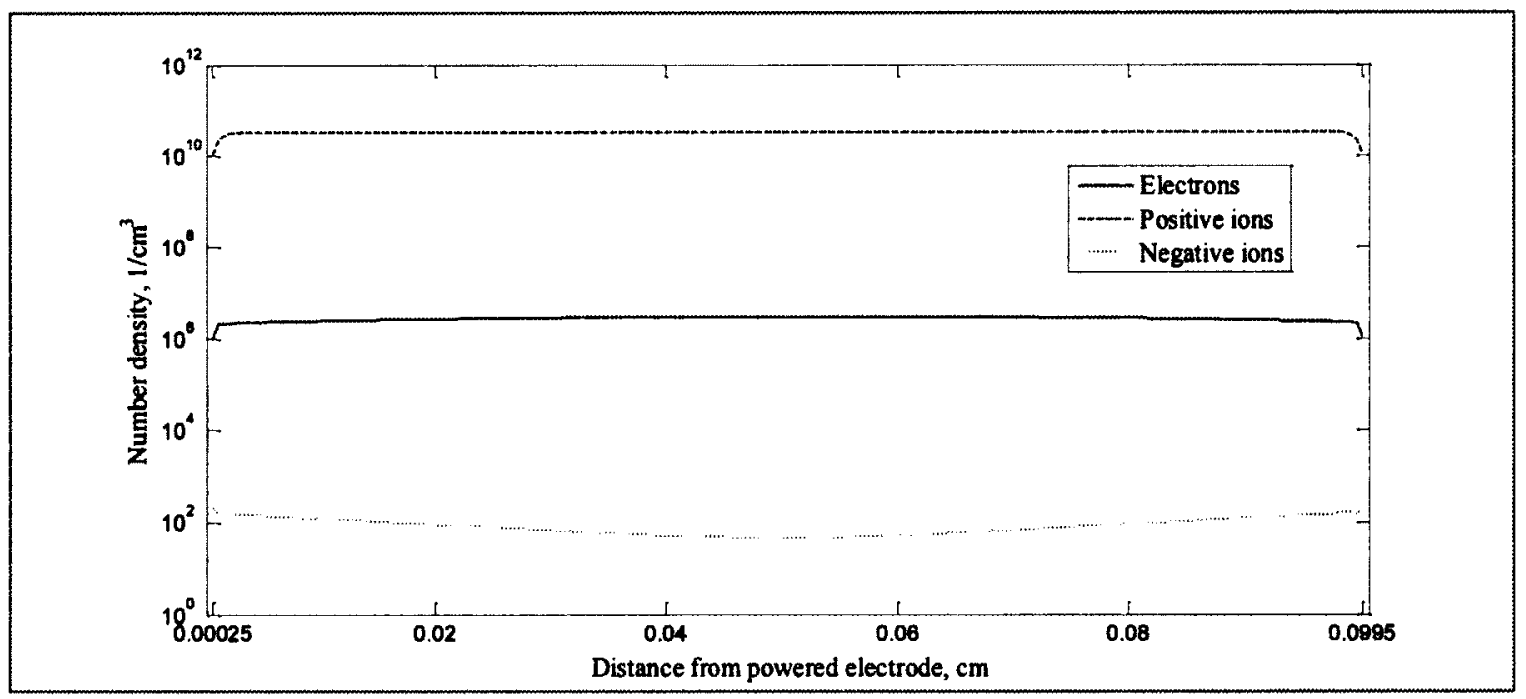

Figure 9.6a: Number density of species of the RF discharge at 1 atm, averaged over the 100th cycle 
As illustrated in Figure 9.6b, the positively charged plasma is sustained in relatively low and linear electric field; the electric field magnitude is on the order of $10^{3} \mathrm{~V} / \mathrm{cm}$ near the electrodes. The electron mean temperature is mostly around $1 \mathrm{eV}$, and peaks around $2 \mathrm{eV}$ near the powered electrode. Recall that the theory of electric discharges and the breakdown simulations illustrate low electric field non-uniformity on the order of $10^{3}$ $\mathrm{V} / \mathrm{cm}$ prior to breakdown. The glow discharge has much higher distorted fields as will be shown next. This suggests that the discharge simulated here is a RF corona, since the cathode does not emit enough secondary electrons to sustain a quasi-neutral plasma.

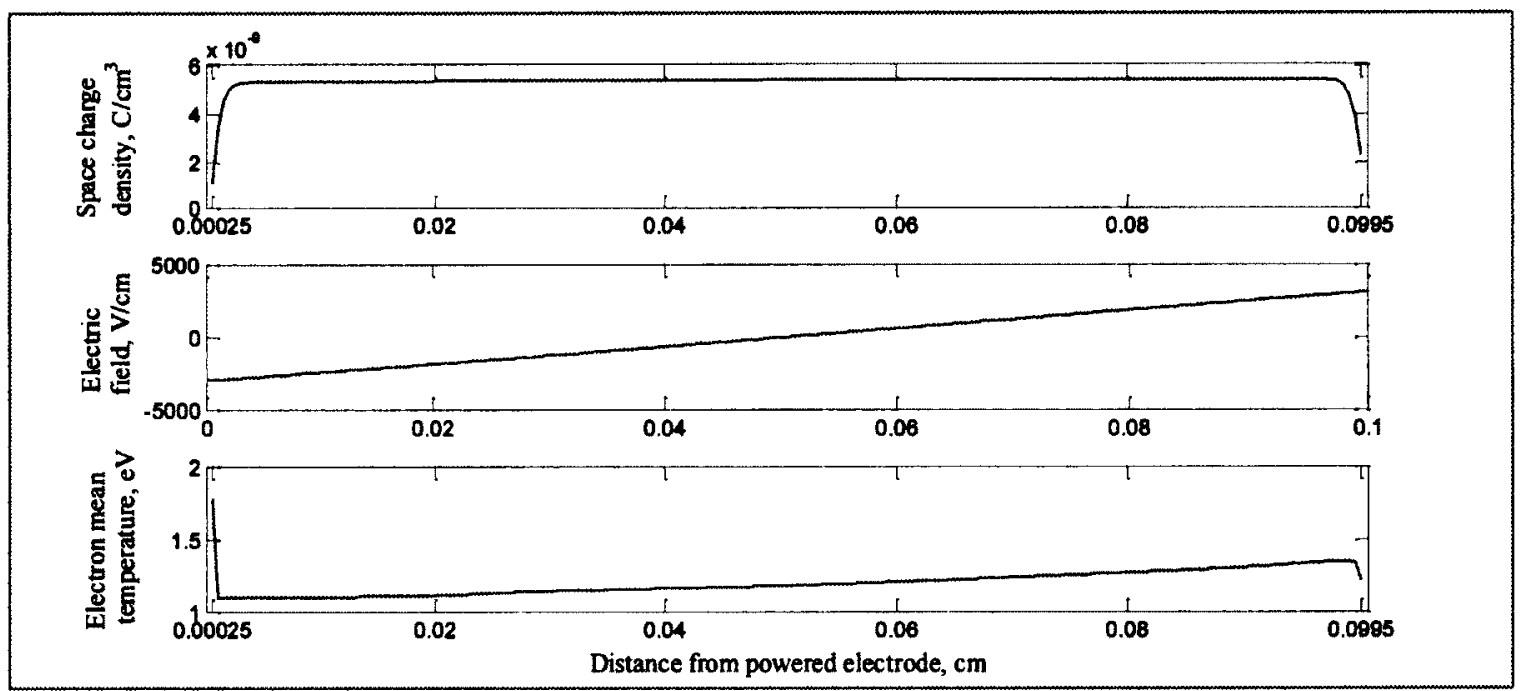

Figure 9.6b: Spatial space charge, electric field, and electron temperature of the RF discharge at 1 atm, averaged over the 100 th cycle

Figure 9.6c indicates that the voltage and current waveforms are stabilizing for the plasma distribution shown in Figures 9.6a. It should be noted that the capacitive phaseshift in Figure 9.6c resembles the second mode of discharge observed experimentally (see Chapter 6). A direct correlation cannot be made since the simulated pressure is $1 \mathrm{~atm}$, at 13.56 MHz frequency, whereas the experimentally observed transition in SPDI occurs at 
110 psi gauge and $35.7 \mathrm{kHz}$ frequency. Nevertheless, it is possible that the second mode of discharge observed in SPDI is an AC corona.

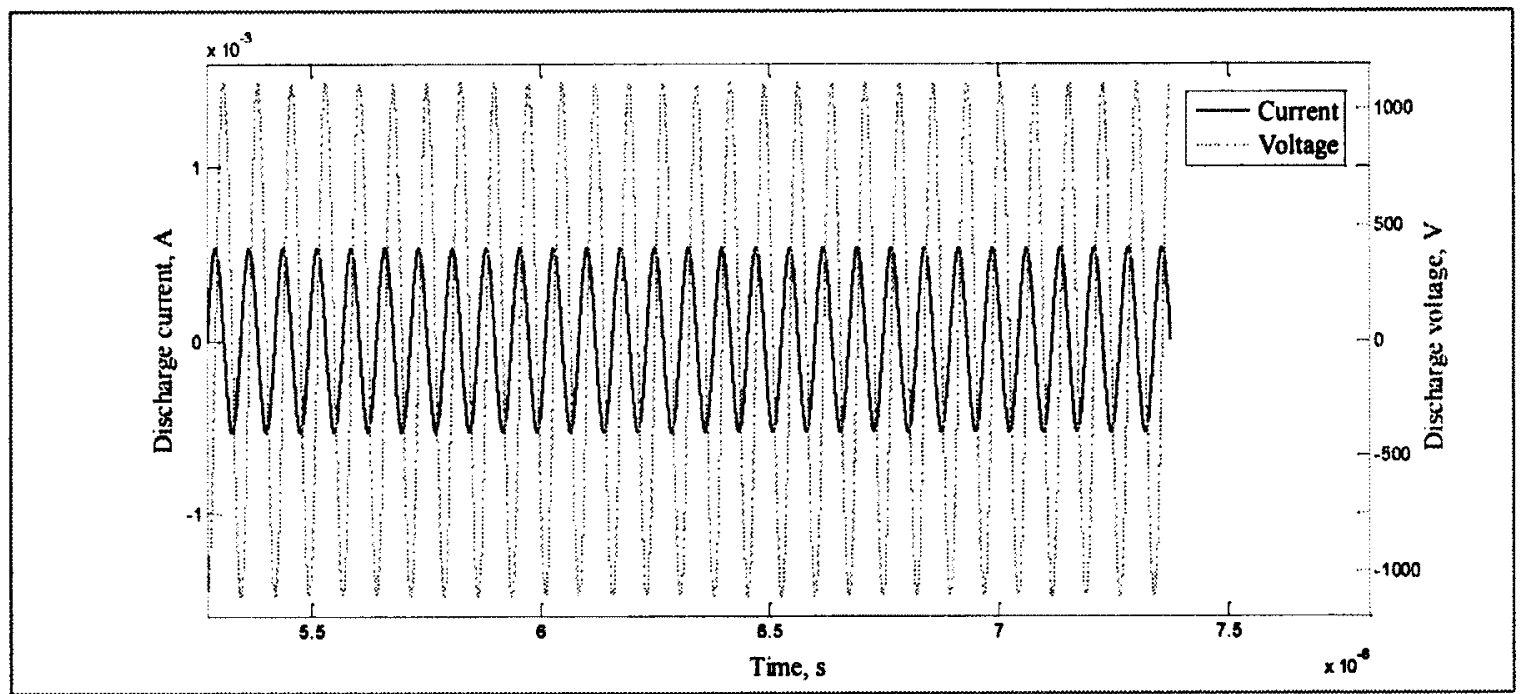

Figure 9.6c: Discharge voltage and current of the RF discharge at 1 atm, approaching the 100 th cycle

The $35.7 \mathrm{kHz}$ discharge, such as the plasma support phase in SPDI, was not simulated due to the computational demand. It should be noted that because of SPDI's low frequency relative to the ion frequency, the discharge is harder to stabilize since for every polarity switch plasma is turned off. On the contrary, for $\mathrm{MHz}$ frequency discharges only electrons respond to the electric field oscillations.

Recall that SPDI's plasma support phase in the first mode was shown to be a $\gamma$-mode glow discharge, where the impedance is mostly resistive (see Chapter 6). Therefore, we assume that the sheath kinetics in SPDI's glow discharge resemble the transient DC glow discharge, which is simulated next. 


\subsubsection{Plasma distributions in transient glow DC discharges}

The following results are transient distributions for $5 \mu \mathrm{s}$ DC discharge at pressures of 1,3 and $5 \mathrm{~atm}$. This is an estimate of the DC period of the plasma support phase illustrated in Figure 6.4. The same DC supply used for breakdown is used here. Again, the resistance of the external circuit is set to $100 \Omega$.

Figure 9.7a illustrates the transient plasma distribution after $5 \mu \mathrm{s}$ at $1 \mathrm{~atm}$. The cathode sheath near the right electrode is evident due to electron depletion. Its thickness is around $160 \mu \mathrm{m}$.

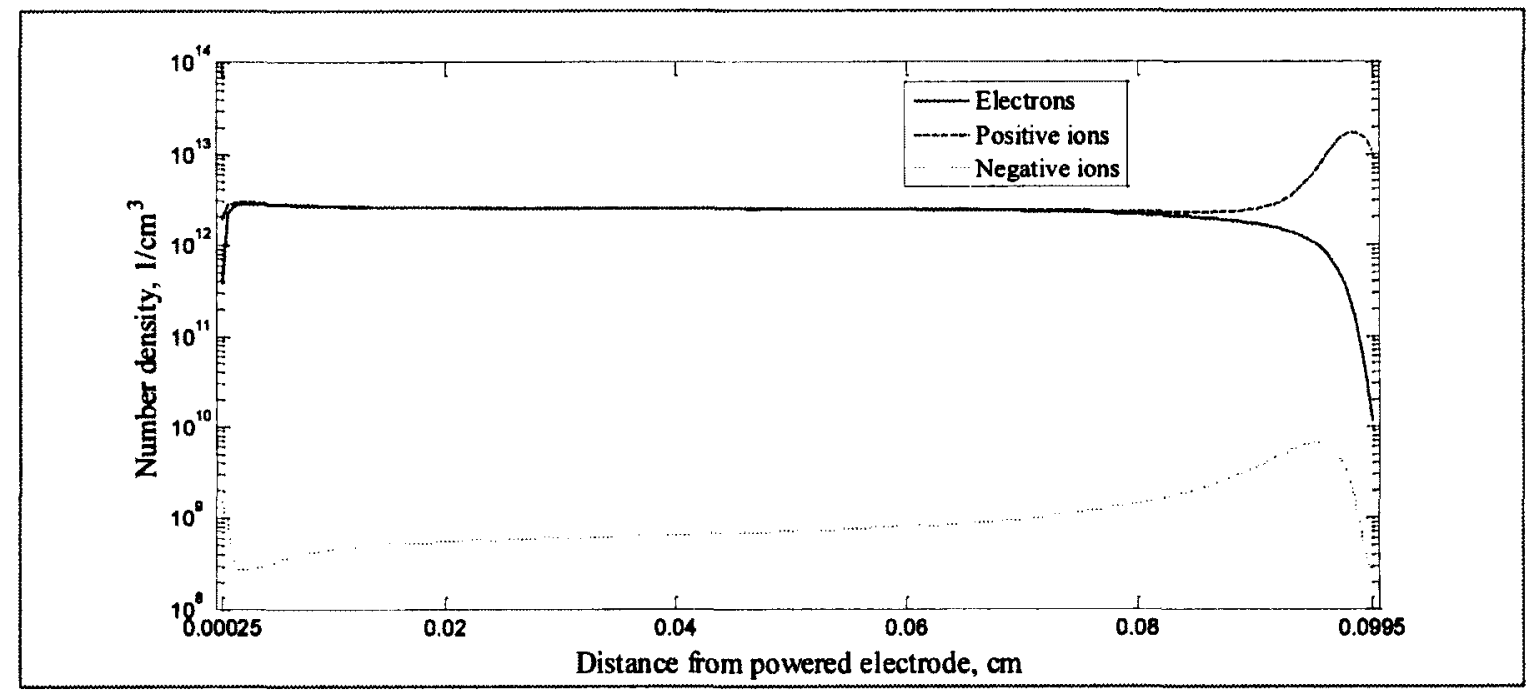

Figure 9.7a: Number density of plasma species for the transient DC glow at 1 atm

However, the anode sheath is not observed since the simulation has not reached its quasisteady state. Sufficient time must be allowed to form the positive ion depleted region. The plasma density is approximately $2.5 \cdot 10^{12} \mathrm{~cm}^{-3}$. Figure $9.7 \mathrm{~b}$ illustrates that the electric field is only uniform in the plasma bulk (approximately $1.3 \cdot 10^{4} \mathrm{~V} / \mathrm{cm}$ ), hence the discharge is approaching quasi-neutrality due to ambipolar diffusion. It should be noted 
that in quasi-steady state, the field is expected to decrease to about $10 \mathrm{~V} / \mathrm{cm}$. The highest electric field is $1.7 \cdot 10^{5} \mathrm{~V} / \mathrm{cm}$ and occurs at the cathode due to the positive space charge in the sheath. Also shown in Figure 9.7b is that electron temperatures are around $1.3 \mathrm{eV}$ in the plasma bulk, and peak near the electrodes. This is due to the collision-free nature of the sheath.

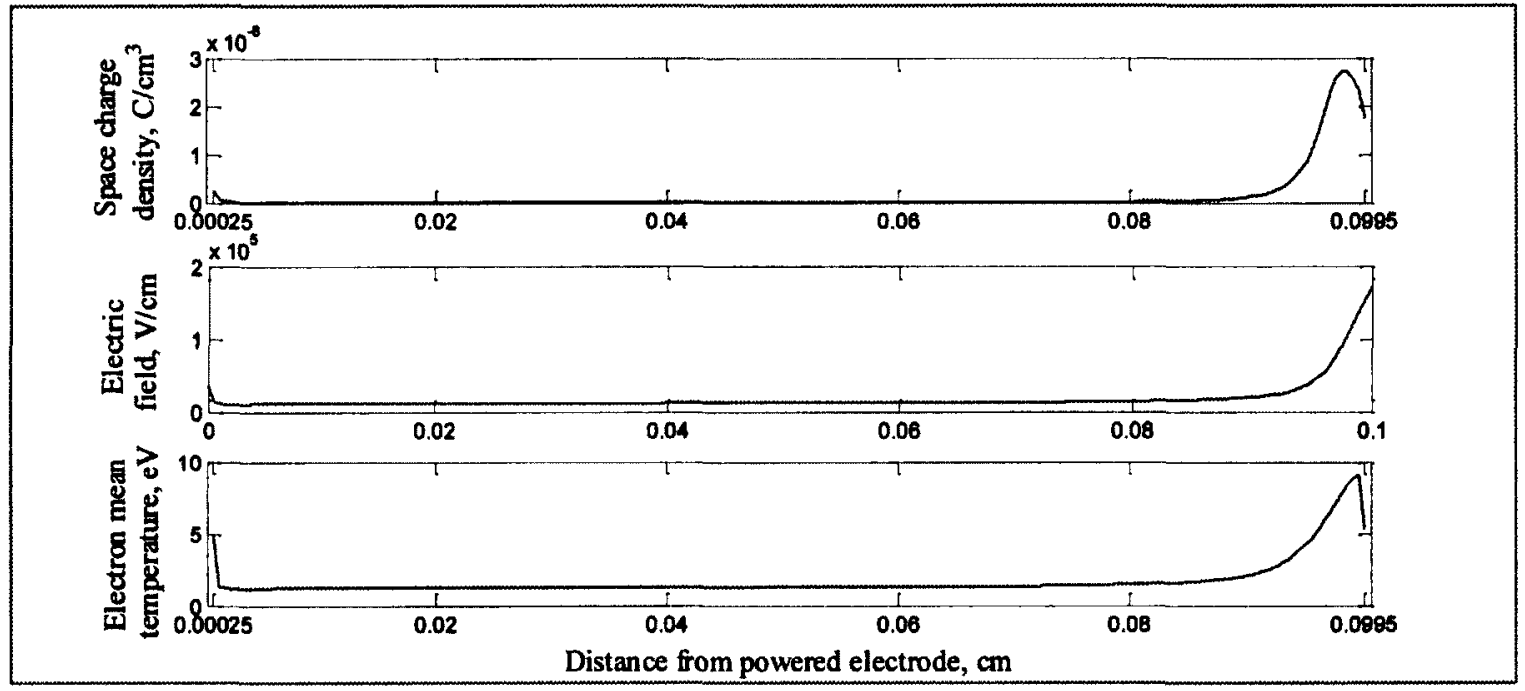

Figure 9.7b: Spatial space charge, electric field, and electron temperature for the transient DC glow at 1 atm

In Figure 9.7c, the discharge voltage and current are shown to stabilize at approximately $33 \mathrm{~W}$ discharge power; this value is obtained by multiplying the discharge voltage and current in Figure $9.7 \mathrm{c}$ at $5 \mu \mathrm{s}$. 


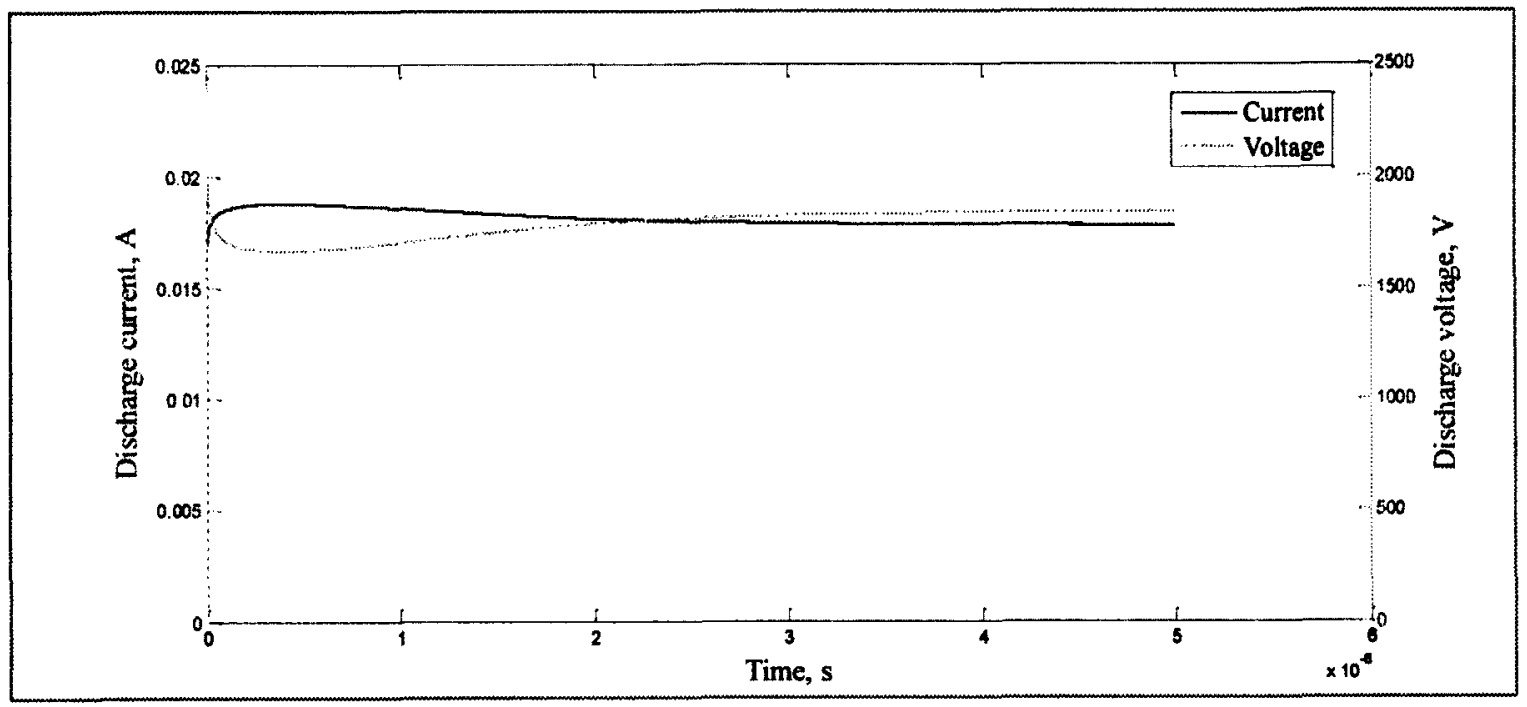

Figure 9.7c: Discharge voltage and current for the transient DC glow at 1 atm

Figure 9.8a illustrates the plasma species distribution after $5 \mu \mathrm{s}$ at $5 \mathrm{~atm}$ pressure.

Similarly to the atmospheric pressure discharge, the cathode sheath is evident and the anode sheath is not formed yet. However, the sheath thickness reduces significantly to 70 $\mu \mathrm{m}$. Quasi-neutrality is observed again in the plasma bulk region, but the plasma density (approximately $1.5 \cdot 10^{13} \mathrm{~cm}^{-3}$ ) increases an order of magnitude at this pressure.

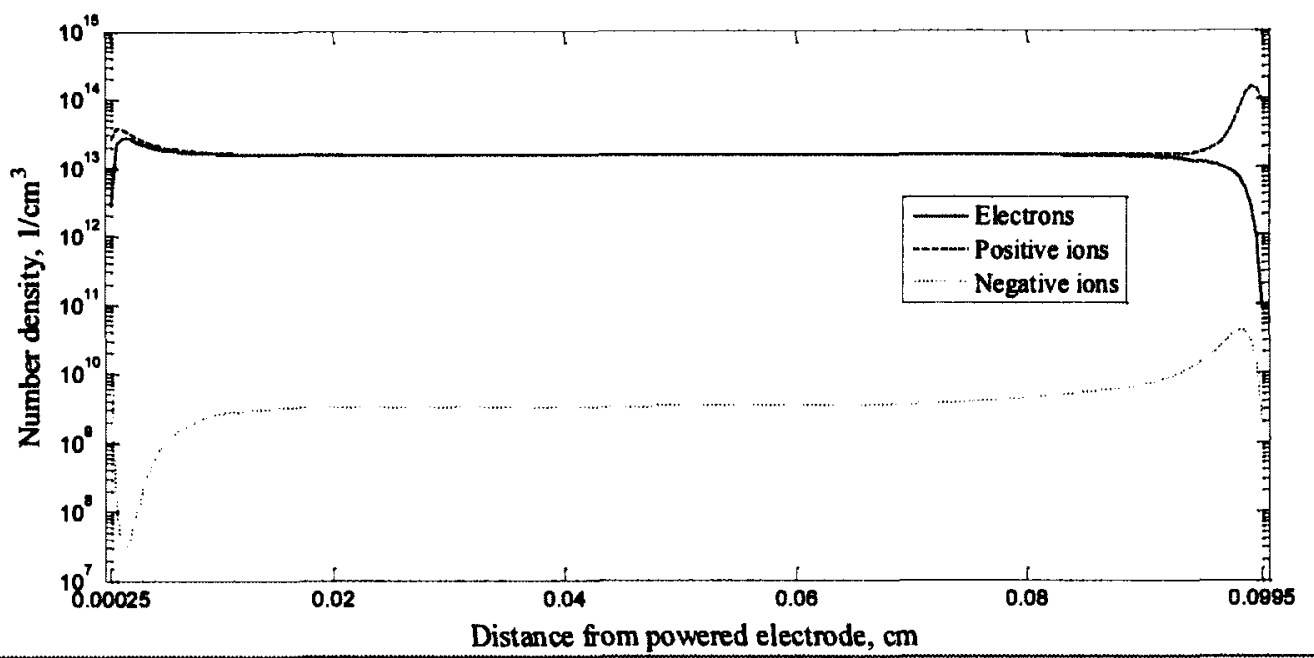

Figure 9.8a: Number density of plasma species for the transient DC glow at $5 \mathrm{~atm}$ 
Distributions of the space charge, electric field and electron temperature at 5 atm resemble the distributions at $1 \mathrm{~atm}$ (see Figure $9.8 \mathrm{~b}$ ). Electric field peaks at $7 \cdot 10^{5} \mathrm{~V} / \mathrm{cm}$ at the cathode due to a larger space charge field at $5 \mathrm{~atm}$ compared to $1 \mathrm{~atm}$. In the plasma bulk, the electric field is approximately $6.3 \cdot 10^{4} \mathrm{~V} / \mathrm{cm}$, and is expected to reduce once quasi-neutrality is reached. The electron temperature in the plasma bulk is $1.2 \mathrm{eV}$, which is slightly lower than the electron temperature at $1 \mathrm{~atm}$. This is due to a higher collisional rate at $5 \mathrm{~atm}$.

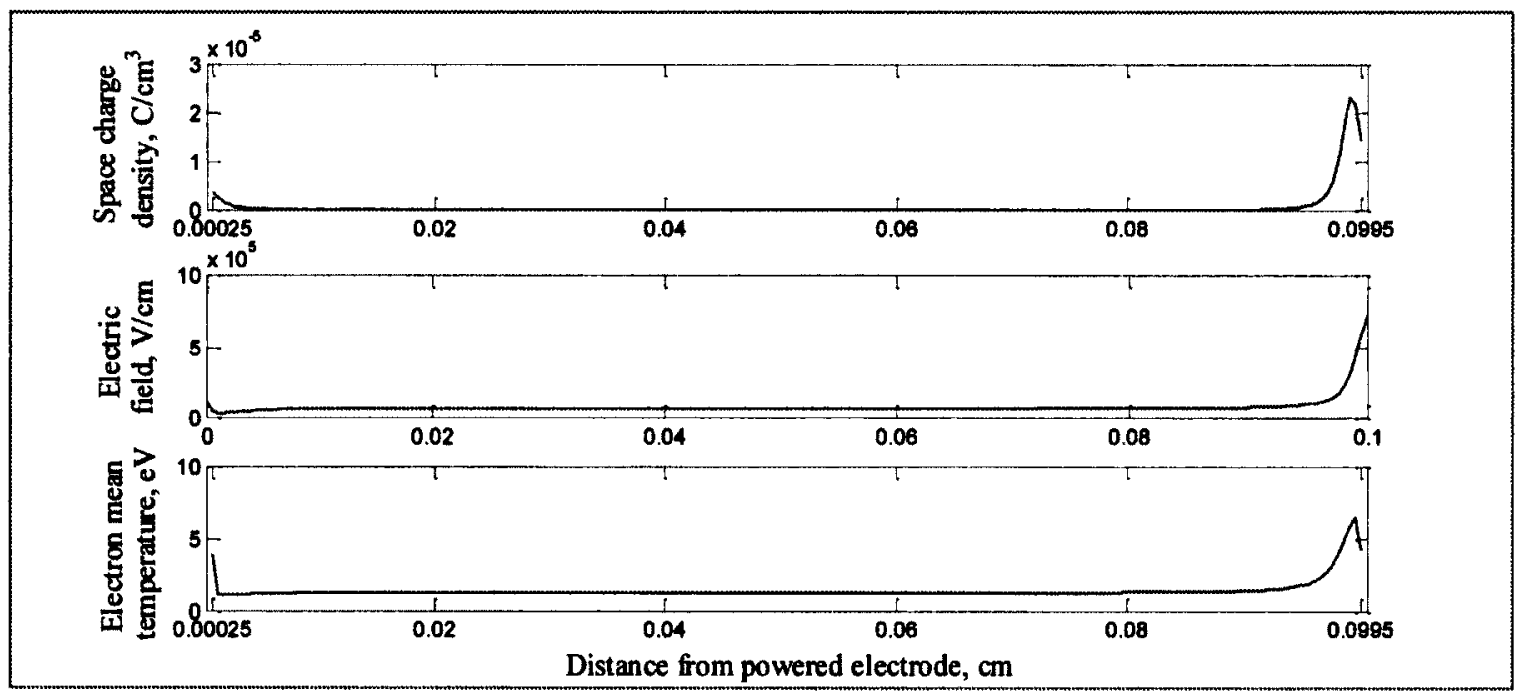

Figure 9.8b: Spatial space charge, electric field, and electron temperature for the transient DC glow at 5 atm

In Figure 9.8c, the discharge voltage and current are shown to stabilize at approximately $796 \mathrm{~W}$ after $5 \mu \mathrm{s}$. Note that this discharge power is not representative SPDI's plasma support phase, since experimental analysis indicates that discharge power remains about $52 \mathrm{~W}$ for pressures up to $8 \mathrm{~atm}$ (equivalent to about $100 \mathrm{psi}$ gauge, see Chapter 6). This indicates the advantage of $R F$ or pulsed-frequency glow discharges: they can be 
stabilized for discharge powers that are 1 to 2 orders of magnitude lower compared to DC glow discharges at equivalent pressure.

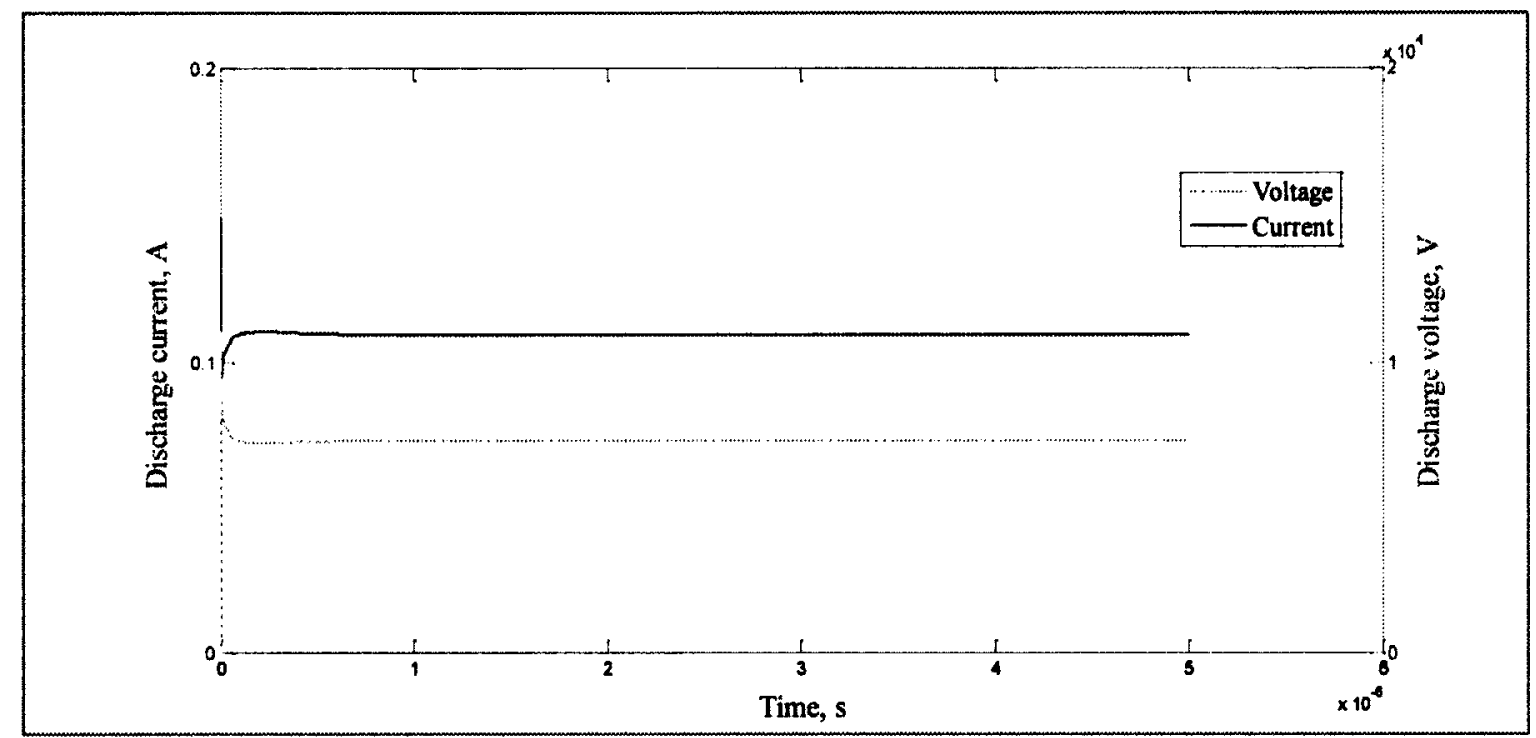

Figure 9.8c: Discharge voltage and current for the transient DC glow at 5 atm

It was found that for pressures above $5 \mathrm{~atm}$, the 3 -moments solver diverges, regardless of the time-step size. This is due to the model being too dissipative; specifically, the electron mean energy does not acquire sufficient energy from the electric field to compensate for collisional energy losses. In order to solve this issue, a more complex reaction mechanism (including molecular dissociation and collisional de-excitation) and gas heating must be taken into account. Alternatively, a 2-moments solver using the local field approximation can be used at high pressures, since quasi-equilibrium distributions are more likely due to high collisional frequency. 
The model in this thesis illustrates that for glow discharges in air at atmospheric and higher pressures, electrons and positive ions have the most important contribution to the formation of the plasma. A summary of the discharge results is shown in Table 9.2.

Table 9.2: Summary of the discharge results for the transient DC simulations

\begin{tabular}{|l|l|l|l|l|}
\hline $\begin{array}{l}\text { Pressue, } \\
{[\mathrm{atm}]}\end{array}$ & $\Delta t_{I N I},[\mathrm{~ns}]$ & $\begin{array}{l}\text { Discharge } \\
\text { power, }[\mathrm{W}]\end{array}$ & $\begin{array}{l}\text { Cathode sheath } \\
\text { thickness, }[\mu \mathrm{m}]\end{array}$ & $\begin{array}{l}\text { Electron mean temperature in } \\
\text { plasma bulk, }[\mathrm{eV}]\end{array}$ \\
\hline 1 & 1 & 33 & 160 & 1.3 \\
\hline 3 & 0.5 & 165 & 100 & 1.2 \\
\hline 5 & 0.1 & 796 & 70 & 1.2 \\
\hline
\end{tabular}

\subsection{ODE'S Behavior using Results of the 1D Model}

Recall the non-dimensional ODE of Chapter 8

$$
\ddot{q}+\kappa \dot{q}-q^{M+2 / M+1}=\sin (\Omega \tau-\phi)
$$

The non-dimensional variables $q, \kappa, \Omega$, and $\tau$ represent charge, collision-frequency, driving frequency, and time respectively; $M$ is a factor that represents the spatial dependence of the sheath charge (for $M=0$, charge is spaced-averaged over the sheath length), and $\phi$ is the capacitive phase shift observed experimentally.

\subsubsection{The sheath and plasma bulk parameters}

Recall that the $5 \mu$ s transient DC sheath is assumed to resemble the sheath in SPDI's plasma support phase. This assumption was validated previously. The parameters used from the transient glow simulations are outlined in Table 9.3. Also recall that the species densities in the plasma bulk are required to determine the conductivity of the discharge. 
Also, the reduced electric field is used to estimate ion mobility using Equation 7.12, whereas electron mobility is estimated by extrapolation from the database for the mean electron temperatures given in Table 9.3.

Table 9.3: Summary of parameters used in the ODE

\begin{tabular}{|c|c|c|c|c|c|c|c|}
\hline \multirow{2}{*}{$\begin{array}{c}\text { Pressure } \\
{[\mathrm{atm}]}\end{array}$} & \multicolumn{5}{|c|}{ Plasma bulk } & Sheath & Potential \\
\cline { 2 - 8 } & $n_{e}\left[\mathrm{~cm}^{-3}\right]$ & $n_{p}\left[\mathrm{~cm}^{-3}\right]$ & $n_{n}\left[\mathrm{~cm}^{-3}\right]$ & $E / N[\mathrm{Td}]$ & $\bar{T}_{e}[\mathrm{eV}]$ & $n_{\text {net }}\left[\mathrm{cm}^{-3}\right]$ & $V_{o}[\mathrm{~V}]$ \\
\hline 1 & $2.5 \cdot 10^{12}$ & $2.5 \cdot 10^{12}$ & $7.5 \cdot 10^{8}$ & 53 & 1.3 & $4.9 \cdot 10^{12}$ & 1838 \\
\hline 3 & $5.3 \cdot 10^{12}$ & $5.3 \cdot 10^{12}$ & $9.0 \cdot 10^{8}$ & 49 & 1.2 & $1.7 \cdot 10^{13}$ & 4515 \\
\hline 5 & $1.5 \cdot 10^{13}$ & $1.5 \cdot 10^{13}$ & $3.4 \cdot 10^{9}$ & 51 & 1.2 & $4.4 \cdot 10^{13}$ & 7286 \\
\hline
\end{tabular}

Since quasi-steady distributions of SPDI's $35.7 \mathrm{kHz}$ glow discharge were not obtained, the net species density in the sheath is averaged over the sheath size; hence $M=0$. The amplitude of the forcing function is assumed to have equivalent potential to the simulated discharge voltage.

\subsubsection{The steady-state solution of the ODE}

The non-linear term in the ODE is replaced by a quasi-linear term, $E_{N}(q)$,

$$
\ddot{q}+\kappa \dot{q}+E_{N}(q)=\sin (\Omega \tau-\phi)
$$

where $E_{N}(q)=-q^{M+2 / M+1}$. Let the forcing function be $f(\tau)=\sin (\Omega \tau-\theta)$ such that $\theta=\phi-\pi / 2$; the $\pi / 2$ phase-shift couples the voltage waveform to the charge waveform, since $\phi$ is the capacitive phase-shift.

Then the steady state solution of the ODE becomes 


$$
q(\tau)=A \sin (\Omega \tau)
$$

where $A$ represents the amplitude ratio of the response (charge) to the forcing function (potential).

\subsubsection{Describing function of the non-linear sheath voltage}

The forcing function $f(\tau)=\sin (\Omega \tau-\theta)$ can be expanded in terms of $q(\tau)$ and $\dot{q}(\tau)$

$$
\begin{gathered}
f=\sin (\Omega \tau) \cos \theta-\cos (\Omega \tau) \sin \Theta \\
f=\frac{1}{A}\left(\cos \theta \cdot q-\frac{\sin \theta}{\Omega} \cdot \dot{q}\right)
\end{gathered}
$$

By taking the Laplace transform $\mathscr{L}\{q(\tau)\}=x(s)$ with 0 initial conditions,

$$
\left(s^{2}+\kappa s\right) x+E_{N}(x)=\frac{1}{A}\left(\cos \theta-\frac{\sin \theta}{\Omega} s\right) x .
$$

The describing function uses Fourier series to transform the non-linear element into a quasi-linear element. For singular non-linearity

$$
E_{N}(x)=N_{1} x
$$

where $N_{1}$ is the describing function of $E_{N}$.

\subsubsection{The characteristic equation of the ODE}

Substituting $s=\Omega i$ for the frequency domain, the characteristics equation of the ODE is given by

$$
-\Omega^{2}+\left(\kappa+\frac{1}{A} \frac{\sin \theta}{\Omega}\right) \Omega i-\frac{1}{A} \cos \theta+N_{1}=0 .
$$

Then, the describing function of the voltage drop across the sheath can be determined

$$
N_{1}=\Omega^{2}-\Omega \kappa \cot \Theta .
$$


It should be noted that $N_{1}$ is implicitly dependent on $A$ since the system is non-linear; however, for the purpose of this section, only the dependence on the phase-shift is of interest. The parameters that are varied are pressure (characterized by the collision frequency $\kappa$ ) and the capacitive phase shift (characterized by $\Theta$ ).

\subsubsection{Results of the sheath's voltage dependence on the phase-shift}

Figure 9.9 illustrates the evolution of the describing function of the sheath voltage, from an entirely resistive to an entirely capacitive discharge. Although simulated voltages and plasma densities represent hypothetical AC glow discharges, the curves in Figure 9.9 postulate that the SPDI transition is due to the voltage drop across the sheath. It can be seen that the describing function of the non-linearity is 4 orders of magnitude larger if the discharge is purely capacitive, as opposed to resistive. The experimental data in Chapter 6 indicate that past the threshold pressure of 110 psi gauge in air, the glow discharge cannot be maintained. According to Figure 9.9, we postulated that the sheath voltage at high pressure in not feasible to sustain the quasi-neutral plasma. 


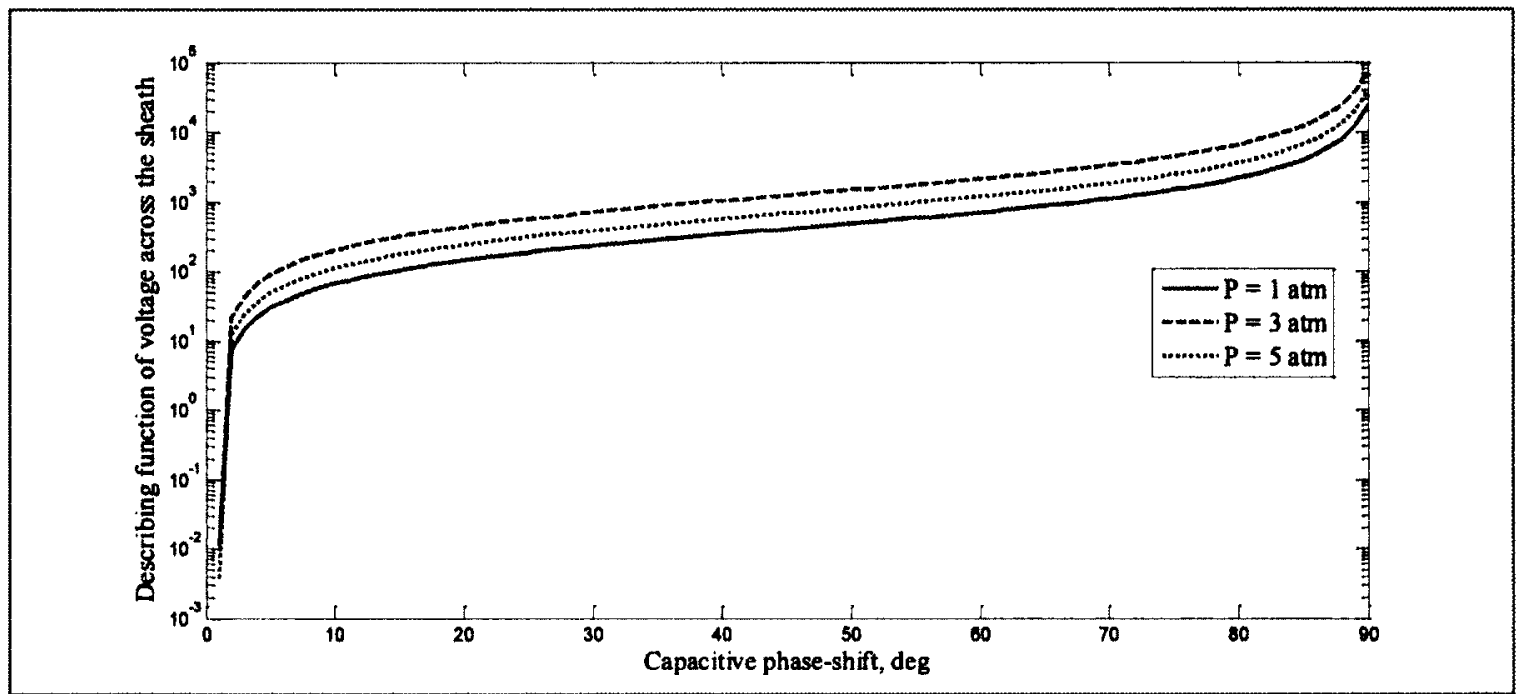

Figure 9.9: Describing function of the sheath voltage for hypothetical discharges at SPDI's frequency

Recall that SPDI's plasma support phase was observed to transition from the quasineutral $\gamma$-mode glow discharge (where $70 \%$ of the discharge power is resistive) to the postulated streamer discharge (where $95 \%$ of the discharge power is capacitive) at the threshold pressure of 110 psi gauge.

According to the ODE model, we postulate that SPDI cannot sustain the glow discharge at very high pressures due to the sheath disappearance. 


\section{Chapter: Concluding Remarks}

The conclusions of this thesis reflect on the experimental and theoretical results describing SPDI plasma, and limitations of the plasma model developed. Recommendations for improving the plasma model and future work regarding SPDI assisted combustion are also discussed.

\subsection{Experimental Results of SPDI Plasma}

The experiments of SPDI discharge in air and SPDI assisted combustion indicate that the plasma support phase undergoes a discharge transition at a pressure threshold. In pressurized air, this transition occurs at 110 psi gauge for a power setting of $44 \mathrm{~W}$. Because the oscilloscope data of the discharge voltage and current waveforms are similar for SPDI plasma in pressurized air or combustion, a better understanding of the plasma in air allows us to make a first-step correlation to SPDI plasma in combustion.

We postulate that the plasma support phase can sustain either an AC glow discharge or an AC corona, depending on the pressure of the medium. Visually, the glow in SPDI resembles a $\gamma$-mode $\mathrm{AC}$ glow discharge, where about $70 \%$ of the power is resistive; in the AC corona mode, where about $95 \%$ of the power is reflected, the light emission cannot be detected by a CCD camera. Furthermore, the transition between the $\gamma$-mode $\mathrm{AC}$ glow and the $\mathrm{AC}$ corona, at the threshold pressure, causes large capacitive impedance and significant DC bias current. 


\subsection{Simulated Results of the RF and AC plasma}

Theoretical results of the plasma model agree qualitatively with the theories of breakdown and the glow discharge. Simulations illustrate that for pressures from atmospheric to $5 \mathrm{~atm}$, the ionization wave velocity toward the cathode is on the order of $10^{6} \mathrm{~cm} / \mathrm{s}$ and the time to breakdown is on the order of $10^{2} \mathrm{~ns}$. However, for higher pressures, the ionization wave velocity toward the cathode is on the order of $10^{7} \mathrm{~cm} / \mathrm{s}$ and the time to breakdown is on the order of $10 \mathrm{~ns}$. Based on these results, the effects of photo-ionization become important for breakdown in air at high pressure; this is directly related to the transition from Townsend to streamer breakdown.

Simulated results of the $13.56 \mathrm{MHz} \mathrm{RF}$ discharge, and the $\mathrm{DC}$ discharge after breakdown illustrate two modes of discharge: an RF corona, and a DC glow. For both, positive ions in air and electrons play key roles in the plasma sustainability. The RF corona at atmospheric pressure forms a positively-charged gap, with uniform densities of positive ions and electrons, of $10^{10}$ and $10^{6} \mathrm{~cm}^{-3}$ respectively. The space-averaged electric field grows linearly from 0 at the gap center to $10^{3} \mathrm{~V} / \mathrm{cm}$ near the electrodes. The discharge power is very low due to insufficient secondary electron emission, thus the current magnitude is on the order of $1 \mathrm{~mA}$. The voltage and current waveforms resemble the oscilloscope waveforms of the postulated AC corona glow in SPDI due to the large capacitive phase-shift; however, no direct connection between these is made since the working frequency of SPDI is only $35.7 \mathrm{kHz}$.

The DC glow discharge in pressures from atmospheric to $5 \mathrm{~atm}$ forms a quasi-neutral plasma that is sustained by the voltage drop in the cathode sheath. The plasma density 
reaches $10^{12} \mathrm{~cm}^{-3}$ and $10^{13} \mathrm{~cm}^{-3}$ in air at a temperature of $25 \mathrm{C}$, and pressure of atmospheric and $5 \mathrm{~atm}$ respectively. In the quasi-neutral region, the electric field remains uniform but relatively large $\left(10^{4} \mathrm{~V} / \mathrm{cm}\right)$ since the quasi-steady state was not reached and the anode sheath was not formed. However, near the cathode, the positive sheath shrinks and the electric field rises from $160 \mu \mathrm{m}$ and $10^{5} \mathrm{~V} / \mathrm{cm}$ at atmospheric pressure, to $70 \mu \mathrm{m}$ and $10^{6} \mathrm{~V} / \mathrm{cm}$ at $5 \mathrm{~atm}$.

The analysis of the non-linear ODE equation used hypothetical AC discharges using the DC simulations above and SPDI's $35.7 \mathrm{kHz}$ frequency. From this analysis, we postulate that the transition from SPDI's AC glow discharge to the AC corona is due to the plasma sheath disappearance, which causes large capacitive impedance.

\subsection{Limitations and Improvements of the Plasma Model}

The main assumptions that limit the accuracy of the plasma model are the highly simplified reaction mechanism and constant gas temperature. Firstly, the reaction mechanism only considers ionization due to electron impact and neglects dissociation reactions and atomic ions which are relevant for discharges in air. Moreover, because collision frequency scales with pressure, it is more difficult to sustain a plasma discharge at high pressures; perhaps a more complex reaction mechanism can overcome this issue. Secondly, assuming constant gas temperature enforces a uniform distribution of air molecules. However, the reduced electric field would increase/decrease locally if gas heating were accounted for. This would create localized regions where electrons are at higher temperature to support more ionization. It was found that the 3-moments solver 
developed in this thesis is too dissipative when the pressure exceeds $5 \mathrm{~atm}$; hence gas heating and collisional de-excitation must be accounted for.

It should be noted that the plasma model developed in this thesis does not provide accurate spatial distributions of plasma species in SPDI since it only considers variations along the axis of discharge. Experimental data and the spark plug geometry indicate that 3-dimensional variations are significant due to the electric field non-uniformity. Furthermore, automotive spark plugs have an insular between the powered electrode and the grounded case to ensure current pathway between the powered and grounded electrodes. In the case of SPDI's plasma support phase, the insular can act as a dielectric barrier, and hence stabilize the glow discharge at higher pressures. It is recommended that a dielectric barrier parallel to the gap is implemented in the circuit model.

\subsection{Improvements in the Computational Time of the Plasma Model}

There are some modifications that can speed up the simulation time. First of all, progression in time is via a while loop, which is computationally unattractive in Matlab. It was found that code converge slows down in time significantly around the polarity change, implying that more time-step iterations were required. Using a language such as $\mathrm{C}++$ would accelerate the overall computational time.

Secondly, an equation of best fit should be used to calculate the electron transport and rate coefficients over electron mean energies applicable to plasmas of interest. Currently, the code interpolates these coefficients from a database, which is computationally more demanding. 
Thirdly, the external circuit should be implemented in SPICE or Matlab's Simulink instead of an M-file solver to update the discharge voltage and current. This can provide a better convergence error for coupling the plasma solver to the external circuit, and probably decrease the number of time-step iterations required.

\subsection{Future Work regarding SPDI Assisted Combustion}

The results of this thesis indicate the non-thermal nature of discharges, such as the plasma produced by SPDI. The next step is to determine the emission spectra of the plasma support phase, and develop a combustion model that monitors the effects of longduration plasma support on the flame propagation. This combustion model must be a combination of numerical and experimental analysis.

In particular, it is suggested that sources of photo-ionization, vibrational - vibrational energy exchange, and multi-site combustion ignition due to streamer discharges are closely looked at. 


\section{Appendix A}

\section{Computing the photo-ionization source more accurately}

Photo-emission intensity can be considered proportional to the local rate of ionization by electron impact, and inversely proportional to the squared distance from the photon source

$$
I_{p h}(x+d x)=A \cdot S_{\text {ioniz }}(x) \cdot h(x) / d x^{2}
$$

In Equation A.1, $S_{\text {ioniz }}(x)=\alpha\left|n_{e} v_{e}\right|$, is the electron impact ionization source in Equations 7.1 and 7.2, $A$ is a proportionality constant; for instance, $A=0.038$ in air as estimated by Quast and Lalic (2009); $h(x)$ is the photo-absorption coefficient.

The photo-absorption coefficient represents the amount of photo-ionization events per photo-absorption (i.e. $h=1$ means that all photons have enough energy for ionization, whereas $h=0$ means that only elastic scattering and internally excited states are produced). Photo-absorption coefficient must be estimated as function of local electric field or degree of ionization. In order to determine the total photo-ionization source at a point in the discharge gap, Equation A.1 must be integrated over the entire gap

$$
S_{p h}(x+d x)=\int_{0}^{d} I_{p h}(x+d x) d x
$$




\section{Appendix B}

\section{Semi-implicit coupling to the electric field}

The semi-implicit coupling is determined by linearizing the local space charge in Equation 7.23 (Poisson equation) in time

$$
\nabla^{2} V^{k+1}=-\frac{e}{\varepsilon_{o}}\left[\left(n_{p}-n_{n}-n_{e}\right)^{k}+\frac{\partial\left(\tilde{n}_{p}-\tilde{n}_{e}-\tilde{n}_{n}\right)^{k}}{\partial t} \Delta t\right]
$$

The RHS term in Equation B. 1 is only an estimate of $n^{k+1}$, where only the electric field is implicit $E^{k+1}$. From Equations 7.1 through 7.3 for the electron/ion density,

$$
\frac{\partial \tilde{n}^{k}}{\partial t}=\nabla \cdot\left(\mp \mu^{k} n^{k} \nabla V^{k+1}+D^{k} \nabla n^{k}\right)+S^{k}
$$

Note that drift velocity was replaced by $\mathbf{v}= \pm \mu \mathbf{E}$, and electric field by $E=-\nabla V$.

If Equation B.2 is plugged into Equation B.1, one obtains

$$
\begin{aligned}
\nabla\left[\varepsilon_{o}+\mathrm{e} \Delta \mathrm{t}\left(\mu_{p}\right.\right. & \left.\left.n_{p}^{k}+\mu_{n} n_{n}^{k}+\mu_{e} n_{e}^{k}\right)\right] \nabla V^{k+1} \\
& =-e\left[\left(n_{p}-n_{n}-n_{e}\right)^{k}\right. \\
& \left.+\Delta \mathrm{t}\left\{\nabla \cdot\left(D_{p} \nabla n_{p}-D_{n} \nabla n_{n}-D_{e} \nabla n_{e}\right)^{k}\right\}\right]
\end{aligned}
$$

The RHS of Equation B.3 can be simplified using the dielectric relaxation time as given by Equation 7.37 ,

$$
t_{D R}^{k}=\frac{\varepsilon_{o}}{\mathrm{e}\left(\mu_{p} n_{p}^{k}+\mu_{n} n_{n}^{k}+\mu_{e} n_{e}^{k}\right)}
$$

Then Equation B.3 reduces to 


$$
\begin{aligned}
\nabla\left(1+\frac{\Delta \mathrm{t}}{t_{D R}{ }^{k}}\right) & \nabla \mathrm{V}^{k+1} \\
& =-\frac{e}{\varepsilon_{o}}\left[\left(n_{p}-n_{n}-n_{e}\right)^{k}\right. \\
& \left.+\Delta \mathrm{t}\left\{\nabla \cdot\left(D_{p} \nabla n_{p}-D_{n} \nabla n_{n}-D_{e} \nabla n_{e}\right)^{k}\right\}\right]
\end{aligned}
$$

In Equation B.4, the particle density and transport coefficients are at time $k$, and only the electric field is at time $k+1$, hence the weak form of coupling. This is why Equation B.4 must be solved with temporarily reduced time-step. 


\section{Appendix C}

\section{Implicit form of the collisional energy exchange}

The total collisional energy exchange is given by Equation 7.6

$$
\sum R U=-3 \frac{e}{\mu_{e} M} n_{e} k\left(T_{e}-T_{g a s}\right)-\sum_{\text {inel }} N n_{e} k_{i}(\bar{\varepsilon}) U_{i}
$$

In Equation C.1, the electron-neutral collision frequency, $\vartheta_{e n}$ has been replaced by $e / m_{e} \mu_{e}$ according to Equation 2.9. Let $E_{R}$ be the elastic energy exchange, and $I_{R}$ be the inelastic energy exchange.

$$
\begin{gathered}
E_{R}=-3 \frac{e}{\mu_{e} M} n_{e} k\left(T_{e}-T_{g a s}\right) \\
I_{R}=-\sum_{\text {inel }} N n_{e} k_{i}(\bar{\varepsilon}) U_{i}
\end{gathered}
$$

It can be seen that the elastic energy exchange depends on the electron mobility, $\mu_{e}$ and electron temperature $T_{e}$, and the inelastic energy exchange depends on the inelastic reaction rate $k_{i}$. Hence these 3 parameters must be linearized with respect to mean electron energy. Thus

$$
\begin{aligned}
\sum R U^{k+1}= & \sum R U^{k}+\frac{\partial \sum E_{R}{ }^{k}}{\partial \mu_{e}}\left(\mu_{e}{ }^{k+1}-\mu_{e}{ }^{k}\right) \\
& +\frac{\partial \sum E_{R}{ }^{k}}{\partial T_{e}}\left(T_{e}{ }^{k+1}-T_{e}{ }^{k}\right) \\
& +\frac{\partial \sum I_{R}{ }^{k}}{\partial k_{i}(\bar{\varepsilon})}\left(k_{i}(\bar{\varepsilon})^{k+1}-k_{i}(\bar{\varepsilon})^{k}\right)
\end{aligned}
$$

The last 3 RHS terms of Equation C. 3 are determined from Equation C.2

$$
\frac{\partial \sum E_{R}}{\partial \mu_{e}}=\frac{3 e n_{e} k\left(T_{e}-T_{g a s}\right)}{\mu_{e}^{2} M}
$$




$$
\begin{gathered}
\frac{\partial \sum E_{R}}{\partial T_{e}}=-\frac{3 e n_{e} k}{\mu_{e} M} \\
\frac{\partial \sum I_{R}}{\partial k_{i}(\bar{\varepsilon})}=-N n_{e} \sum_{\text {inel }} U_{i}
\end{gathered}
$$

Next, $\mu_{e}^{k+1}, T_{e}^{k+1}$ and $k_{i}(\bar{\varepsilon})^{k+1}$ must be linearized with respect to $\bar{\varepsilon}$

$$
\begin{gathered}
\mu_{e}^{k+1}-\mu_{e}^{k}=\left(\frac{\partial \mu_{e}}{\partial \bar{\varepsilon}}\right)\left(\bar{\varepsilon}^{k+1}-\bar{\varepsilon}^{k}\right) \\
T_{e}^{k+1}-T_{e}^{k}=\left(\frac{\partial T_{e}}{\partial \bar{\varepsilon}}\right)\left(\bar{\varepsilon}^{k+1}-\bar{\varepsilon}^{k}\right) \\
k_{i}(\bar{\varepsilon})^{k+1}-k_{i}(\bar{\varepsilon})^{k}=\left(\frac{\partial k_{i}(\bar{\varepsilon})}{\partial \bar{\varepsilon}}\right)\left(\bar{\varepsilon}^{k+1}-\bar{\varepsilon}^{k}\right)
\end{gathered}
$$

At this point, Equation C. 3 can be written as

$$
\begin{aligned}
\sum R U^{k+1}= & \sum R U^{k} \\
& +\frac{3 e n_{e} k\left(T_{e}-T_{g a s}\right)}{\mu_{e}^{2} M}\left(\frac{\partial \mu_{e}}{\partial \bar{\varepsilon}}\right)\left(\bar{\varepsilon}^{k+1}-\bar{\varepsilon}^{k}\right) \\
& -\frac{3 e n_{e} k}{\mu_{e} M}\left(\frac{\partial T_{e}}{\partial \bar{\varepsilon}}\right)\left(\bar{\varepsilon}^{k+1}-\bar{\varepsilon}^{k}\right) \\
& -N n_{e} \sum_{\text {inel }} U_{i}\left(\frac{\partial k_{i}(\bar{\varepsilon})}{\partial \bar{\varepsilon}}\right)\left(\bar{\varepsilon}^{k+1}-\bar{\varepsilon}^{k}\right)
\end{aligned}
$$

Recall that in this model, the discretized variable is the electron energy density, $n_{\varepsilon}$, which is given by Equation 7.7

$$
\bar{\varepsilon}=\frac{n_{\varepsilon}}{n_{e}}
$$

The electron mean energy can be linearized with respect to $n_{e}$ and $n_{\varepsilon}$

$$
\bar{\varepsilon}^{k+1}=\bar{\varepsilon}^{k}+\left(\frac{\partial \bar{\varepsilon}}{\partial n_{\varepsilon}}\right)\left(n_{\varepsilon}{ }^{k+1}-n_{\varepsilon}{ }^{k}\right)+\left(\frac{\partial \bar{\varepsilon}}{\partial n_{e}}\right)\left(n_{e}{ }^{k+1}-n_{e}{ }^{k}\right)
$$




$$
\begin{gathered}
\bar{\varepsilon}^{k+1}=\bar{\varepsilon}^{k}+\frac{1}{n_{e}}\left(n_{\varepsilon}{ }^{k+1}-n_{\varepsilon}{ }^{k}\right)-\frac{n_{\varepsilon}}{n_{e}{ }^{2}}\left(n_{e}{ }^{k+1}-n_{e}{ }^{k}\right) \\
\bar{\varepsilon}^{k+1}=\bar{\varepsilon}^{k}+\frac{1}{n_{e}{ }^{k}}\left(n_{\varepsilon}^{k+1}-n_{e}^{k+1} \bar{\varepsilon}^{k}\right)
\end{gathered}
$$

The final form of the implicit collisional energy exchange is obtained by plugging Equation C.5 into Equation C.4

$$
\sum R U^{k+1}=\sum R U^{k}+D^{k}\left[n_{\varepsilon}^{k+1}-n_{e}^{k+1} \bar{\varepsilon}^{k}\right]
$$

where $D$ is given by

$$
D=\frac{3 e k\left(T_{e}-T_{g a s}\right)}{\mu_{e}^{2} M}\left(\frac{\partial \mu_{e}}{\partial \bar{\varepsilon}}\right)-\frac{3 e k}{\mu_{e} M}\left(\frac{\partial T_{e}}{\partial \bar{\varepsilon}}\right)-N \sum_{\text {inel }}\left(\frac{\partial k_{i}(\bar{\varepsilon})}{\partial \bar{\varepsilon}}\right) U_{i}
$$

The $\left(\frac{\partial \mu_{e}}{\partial \bar{\varepsilon}}\right),\left(\frac{\partial T_{e}}{\partial \bar{\varepsilon}}\right)$ and $\left(\frac{\partial k_{i}(\bar{\varepsilon})}{\partial \bar{\varepsilon}}\right)$ parameters are determined from the model's database by linear extrapolation.

Note that in Equation C.6, the electron density and the mean electron energy density are implicit. Thus the conservation of electron density must be solved prior to solving the conservation of electron mean energy density. 


\section{References}

Akishev, Y. (2007). Plasmas: Introduction and Characterization [PDF slides]. Retrieved from $\mathrm{http} / / / \mathrm{www}$. acteco.org/Acteco/training_torino/1_Akishev_plasma_introduction.pdf

Azooz, A. A., \& Talal, S. K. (2011). Electric Discharge in Pin-Plate Audio Frequency Plasma. Chin. Phys. Lett., 28(11), 115202-115202.

Balcon, N. (2007). Décharges radio fréquence à pression atmosphérique, diagnostic et modèle numérique (Doctoral dissertation, Université Paul Sabatier-Toulouse III).

Bellenoue, M., Labuda, S. A., \& Engles, M. (2005). Corona discharge ignition and combustion promotion of methane/air mixtures. Proceedings of the European Combustion Meeting, 207, 16.

Braithwaite, N. S. J. (2000). Introduction to gas discharges. Plasma Sources Science and Technology, 9(4), 517.

Capitelli, M., Ferreira, C. M., Gordiets, B. F., \& Osipov, A. I. (2000). Plasma kinetics in atmospheric gases (Vol. 31). Springer.

Czarnetzki, U., Mussenbrock, T., \& Brinkmann, R. P. (2006). Self-excitation of the plasma series resonance in radio-frequency discharges: An analytical description. Physics of plasmas, 13, 123503.

Elert, G. (2012). Ignition Temperature of Gasoline. Retrieved from http:/hypertextbook.com/facts/2003/ShaniChristopher.shtml

Farouk, T. I. (2009). Modeling and simulations of DC and RF atmospheric pressure non-thermal micro plasma discharges: Analysis and applications (Doctoral dissertation, Drexel University).

Farouk, T., Farouk, B., Staack, D., Gutsol, A., \& Fridman, A. (2006). Simulation of dc atmospheric pressure argon micro glow-discharge. Plasma Sources Science and Technology, 15(4), 676.

Frensley, W. Scharfetter-Gummel Discretization Scheme for Drift-Diffusion Equations, 2004. Preprint.

Fridman, A. (2008). Plasma chemistry. Cambridge University Press.

Fridman, A., \& Kennedy, L. A. (2004). Plasma physics and engineering. CRC. 
Gallagher, J. W., Brion, C. E., Samson, J. A. R., \& Langhoff, P. W. (1988).Absolute cross sections for molecular photoabsorption, partial photoionization, and ionic photofragmentation process (No. PB-89-186464/XAB). Joint Inst. for Lab. Astrophysics, Boulder, CO (USA).

Giordano, N. A. E. (2012). Investigation of Plasma Assisted Combustion in an Internal Combustion Engine (Master dissertation, Carleton University).

Hagelaar, G. J. M. (2000). Modeling of microdischarges for display technology. Technische Universiteit Eindhoven.

Hagelaar, G. J. M. (2008). Modelling methods for low-temperature plasmas.document de synthèse pour l'obtention de l'Habilitation à Diriger des Recherches, Université de Paul Sabatier, Toulouse.

Hagelaar, G. J. M. \& Pitchford, L. C. (2005). "Solving the Boltzmann equation to obtain electron transport coefficients and rate coefficients for fluid models", Plasma Sci Sources and Tech 14, 722.

Heil, B. G., Brinkmann, R. P., \& Czarnetzki, U. (2008). A hybrid, one-dimensional model of capacitively coupled radio-frequency discharges. Journal of Physics D: Applied Physics, 41(22), 225208.

Kim, S. J., Chung, T. H., \& Bae, S. H. (2009). Characteristic study of atmospheric pressure microplasma jets with various operating conditions. Thin Solid Films, 517(14), 4251-4254.

Koh, W. H., \& Park, I. H. (2003). Numerical simulation of a pulsed corona discharge plasma. JOURNAL-KOREAN PHYSICAL SOCIETY, 42, 920-924.

Kushner, M. J. (2012). EECS 517/NERS 578: Physical Processes in Plasmas, Relation Between Cross Section, Flux, Rate of Collision, and Rate Coefficient. [Class handout]. Retrieved from http://uigelz.eecs.umich.edu/classes/eecs517_2012/eecs517_2012.html

Lawrence Berkley National Laboratory. (2012). Electromagnetic spectrum. Retrieved from http://www.lbl.gov/MicroWorlds/ALSTool/EMSpec/EMSpec2.html

Lieberman, M. A., \& Lichtenberg, A. J. (1994). Principles of Plasma Discharges. Materials Processing ed Wiley (New York).

Liu, J., Wang, F., Lee, L., Theiss, N., Ronney, P., \& Gundersen, M. (2004). Effect of discharge energy and cavity geometry on flame ignition by transient plasma. ALAA Paper, 1011, 5-8.

Lymberopoulos, D., \& Economou, D. (1996). Two-dimensional self-consistent radio frequency plasma simulations relevant to the gaseous electronics conference RF reference cell. J. Res. Nat. Inst. Stand. Technol. v100 i4, 473-494. 
Mardiguian, M. (2009). The Electrostatic Discharge Phenomenon. Electrostatic Discharge: Understand, Simulate, and Fix ESD Problems, Third Edition, 1-38.

Moore, J., \& Siljak, D. (1967). Parameter plane analysis of forced nonlinear oscillations.

MORGAN database, http://www.lxcat.laplace.univ-tlse.fr, retrieved 2012.

Naidis, G. V. (1999). Modelling of plasma chemical processes in pulsed corona discharges. Journal of Physics D: Applied Physics, 30(8), 1214.

Naidis, G. V. (1999). Simulation of streamer-to-spark transition in short non-uniform air gaps. Journal of physics D: Applied physics, 32(20), 2649.

Owaid, A. (2011). Area under a curve calculation. MATLAB File exchange.

Polak, L. S., \& Lebedev, Yu. A. (1998). Plasma chemistry (Ed.). Cambridge International Science Publishing.

Quast, M., \& Lalic, N. R. (2009). Streamer Propagation in a Point-to-Plane Geometry. In Excerpt from the Proceedings of the COMSOL Conference.

Rosocha, L. A., Kim, Y., \& Stange, S. (2004). Application of a non-thermal plasma to combustion enhancement (No. LA-UR-04-6890). Los Alamos National Laboratory.

Roth, J. R. (1995). Industrial Plasma Engineering, Principles, vol. 1. London, UK: Inst. Phys. Publishing.

Rutscher, A. (2008). Characteristics of Low-Temperature Plasmas Under Nonthermal Conditions-A Short Summary. Low Temperature Plasma Physics.

Sakiyama, Y. (2010). Low Temperature Plasma Modeling \& Simulation and Applications [PDF slides]. Retrieved from http://graveslab.cchem.berkeley.edu/upload/ICOPS_minicourse_Yuki.pdf

Shi, J. J., \& Kong, M. G. (2005). Mechanisms of the $\alpha$ and $\gamma$ modes in radio-frequency atmospheric glow discharges. Journal of applied physics, 97(2), 023306-023306.

Singleton, D., Pendleton, S. J., \& Gundersen, M. A. (2010). The role of non-thermal transient plasma for enhanced flame ignition in C2H4-air. Journal of Physics D: Applied Physics, 44(2), 022001 .

Slotine, J. J. E., \& Li, W. (1991). Applied nonlinear control (Vol. 199, No. 1). Englewood Cliffs, NJ: Prentice-Hall. 
Smith, D. J., McMeekin, S. G., Stewart, B. G., \& Wallace, P. A. (2011, June). A numerical model for the electrical breakdown of air within a gap under standard atmospheric conditions: Onedimensional versus two-dimensional approach. In Electrical Insulation Conference (EIC), 2011 (pp. 387-391). IEEE.

Starikovskii, A. Y. (2005). Plasma supported combustion. Proceedings of the Combustion Institute, 30(2), 2405-2417.

Tritt, T. M. (Ed.). (2005). Thermal conductivity: theory, properties, and applications. Springer.

Van Veldhuizen, E. M., \& Rutgers, W. R. (2001). Corona discharges: fundamentals and diagnostics. Invited paper, Proc. Frontiers in Low Temp. Plasma Diagn. IV, Rolduc, Netherlands, 40-49.

Versteeg, H. K., \& Malalasekera, W. (2007). An introduction to computational fluid dynamics: the finite volume method. Prentice Hall.

Wagner, H. E., \& Brandenburg, R. (2011). Atmospheric Pressure Plasmas I [PDF slides]. Retrieved from http://pams2011.physik.uni-greifswald.de/brandenburg_pams11.pdf

Williams, L. (2012). Physics 42: Simple Derivation of Electromagnetic Waves from Maxwell's Equations. [Class handout]. Retrieved from http://www.santarosa.edu/ lwillia2/42/WaveEquationDerivation.pdf

Yang, X., Moravej, M., Nowling, G. R., Chang, J. P., \& Hicks, R. F. (2005). Operating modes of an atmospheric pressure radio frequency plasma. Plasma Science, IEEE Transactions on, 33(2), 294-295.

Zevenhoven, R. (2001). Non-ideal gases in diesel engine processes. Carbon, 147, 126. 